\title{
Using Bayesian Framework to Calibrate a Physically Based Model Describing Strain-Stress Behavior of TRIP Steels
}

\author{
P. Honarmandi ${ }^{a,{ }^{*}}$ and R. Arroyave ${ }^{a}$ \\ ${ }^{a}$ Department of Materials Science and Engineering, Texas A\&M University, College Station, Texas, USA
}

* Corresponding author:

Pejman Honarmandi

hona107@tamu.edu

Tel: +1(979) 845-0750

Fax: +1(979) 862-6835

Postal Address:

Texas A\&M University

Department of Materials Science and Engineering, 3003 TAMU,

College Station, Texas 77843-3003 USA 


\section{Introduction}

The requirements for better manufacturability, higher safety, and lower fuel consumption in the automotive industry have led to the development of advanced materials, such as TRIP steels [1-4]. Generally, TRIP steels are considered as composite steels that contain different phases: ferrite as the matrix with bainite, retained austenite, and/or martensite inclusions $[5,6]$. The presence of retained austenite in the microstructure of these low-alloy steels can give an enhanced combination of strength and fracture toughness. This results from high strain hardening that occurs during plastic deformation by strain-induced martensitic transformation (SIM) [7-10]. SIM behavior is directly related to the stability of the retained austenite and mainly influenced by its volume fraction, grain size, and carbon content [6].

Either to rationalize observations or to assist in the design of novel TRIP steel alloying and thermosmechanical processing protocols it is necessary to develop physically motivated models that account for the effects of phase and chemical constitution on plastic deformation [11]. However, to date, work to address this challenge is rather limited [12] due to the modeling complexities that arise when considering the contribution of different phases to the overall mechanical response of these complex microstructures, in addition to phenomena associated with SIM.

In this regard, Bouquerel et al [13] proposed a physical micromechanical method based on Mecking-Kocks theory for plastic flow behavior of each individual microstructural phase, and combined them using a Gladman-type mixture law. Moreover, they used a modified Olsen-Cohen approach to model SIM [13]. Perlade et al [14] also suggested another physically-based model that considers the effects of morphology and chemical composition of each phase on stress-strain behavior, and subsequently homogenized the flow behavior of all the phases using the iso-work hypothesis. A generalized Olsen-Cohen was also applied to treat SIM in that work [14]. In some other related works, dual or multi-phase microstructures with phase volume fraction evolutions have 
been assumed in the framework of constitutive models [12] or mean field approaches [11, 15] in order to predict stress-strain partitioning within microstructural phases. In addition, there is a lot of work for describing the mechanical behavior of structural materials undergoing SIM, pioneered by Zackay et al [16], Olsen-Cohen [17], and Olson and Azrin [18]. Among recent works, Tomita's constitutive equation in terms of applied stress, strain rate and temperature [19], Thibaud's phenomenological approach [20], and Han's kinetic model [4] based on martensitic variant selection through the Kurdjumov-Sachs orientation relationship are good examples of SIM modelling investigations.

The examples of physically motivated mechanical models provided above, as well as many others not discussed in this work for lack of space, are always subject to limitations inherent in the theoretical and simulation frameworks used, as well as in the uncertainty associated with the experimental observations used to parameterized and/or validate such models. Models are naturally limited in that it is usually the case that not all relevant physical phenomena are accounted for in a given formulation. Further limitations arise from the need to compromise between the need for facile implementation and execution of such models versus the need to accurately represent the microstructural features ultimately responsible for the mechanical behavior of the systems under study-i.e., the tension between analytical vs microstructure-sensitive approaches. Further limitations arise from limitations in the experimental observations themselves, as it is often the case that a considerable fraction of the inputs to the models are in turn determined by characterization techniques that are either limited in their accuracy/resolution or that are costly in resources.

Most approaches to model calibration (parameterization)/validation either do not account for uncertainties in the models and/or experiments and treat the exercise as a purely deterministic one. While such an approach provides valuable insight into the qualitative response of a physically-based model, the neglect of Uncertainty Quantification limits the power of the models as it is not possible 

makes it harder to determine in a quantitative manner the value derived from specific experimental observations either based on whether such observations can be used to reduce the model and/or experimental uncertainty. Additionally, neglect of UQ makes it difficult to deploy the models in materials design/optimization frameworks that account for uncertainty—e.g. robust design. Finally, we would like to note that UQ can be used to estimate values for physical features/parameters of a system that are difficult to determine experimentally.

In the context of UQ, the relevant uncertainties can result from different sources: 1) inherent uncertainty of a physical system, 2) model parameter uncertainty due to lack of knowledge/data about parameters, 3) propagated uncertainty in the case of a sequence of models, and 4) model structure uncertainty owing to the lack of physics in the models. Among these uncertainties, the quantification of the model uncertainty is usually the hardest one because of limited knowledge about the physical systems and inaccurate experimental data. This uncertainty quantification includes the model calibrations with data [21], which is often disregarded or performed superficially in most materials modelling.

It has been found [22] that the Bayesian approach based on Markov Chain Monte Carlo (MCMC) techniques is highly robust compared to other methods for parameter estimation. In fact, MCMC methods resolve the issue associated with the parameter calibration of complex non-linear models, which usually fail using traditional statistical methods [23]. In the case of multi-level models, Browne and Draper [24] indicated that the MCMC-based Bayesian approaches can provide better calibration compared to different likelihood-based methods, i.e., maximum likelihood (ML), marginal quasi-likelihood (MQL), penalized quasi-likelihood (PQL), and restricted maximum likelihood (REML). 
In the current computational work, Rivera [25] and Haidemenopoulos [26] models have been applied to simulate the plastic flow behavior of multiple phases and SIM within dispersed retained austenite, respectively, to account for the mechanical deformation of multi-phase TRIP steel. In the first model, the dislocation density evolution for any individual phase is determined using a thermostatistical theory of plasticity, and overall plastic flow behavior has subsequently been estimated through an iso-work approximation. This approximation realizes the coupling among multiple phases by assuming that the work of deformation is equally partitioned among all phases in the microstructure at any strain interval. The model that accounts for SIM in turn can be considered as a modified Olson-Cohen model for the distribution of nucleation site potency, which predicts the SIM behavior in terms of austenite particle size, austenite chemical composition, and stress triaxiality. These models are applied as case studies for the introduction of Bayesian parameter calibration based on Metropolis-Hastings MCMC algorithm, which is our main direction in this paper.

\section{Models and Methodology}

\subsection{Microstructural Evolution during Plastic Deformation}

In this model, shear stress for each microstructural phase $(i)$ is defined in terms of the contribution of the following mechanisms [27]:

$\tau_{i}=\tau_{0}+\tau_{s}+\tau_{b}+\sqrt{\tau_{i n}^{2}+\tau_{p}^{2}}$

where $\tau_{0}, \tau_{s}, \tau_{b}, \tau_{i n}$, and $\tau_{p}$ are Peierls force, solid solution strengthening, long-range back stress, dislocation strengthening, and precipitation strengthening contributions within phase $i$, respectively. Here, $\tau_{0}$ and $\tau_{s}$ are dependent on chemical compositions of each phase through the existing empirical equations [28-30]. The following relationships have also been proposed for $\tau_{b}$ and $\tau_{p}$ [27]:

$\tau_{b}=\frac{\mu b}{D} n=\frac{\mu b}{D} n^{*}\left(1-\exp \left(-\frac{\lambda}{b n^{*}} \gamma\right)\right)$ 
$\tau_{p} \cong 0.6 \mu b \frac{\sqrt{f_{v}}}{R}$

In which $\mu$ is the shear modulus, $b$ is the Burger's vector magnitude, $D$ is the grain size, $\mathrm{n}$ is the evolution of piled-up dislocations at the grain boundaries in terms of shear strain $(Y), n^{*}$ is the maximum possible number of piled-up dislocations, $\lambda$ is the average spacing between slip planes at the grain boundaries, $f_{v}$ is the precipitate volume fraction, and $R$ is the mean radius of precipitates. $\tau_{\text {in }}$ is defined by Rivera's model [25] based on dislocation density evolution of individual phases during plastic deformation which is described as the difference between the dislocation generation and annihilation rates according to Kocks and Mecking theory [31]:

$\frac{d \rho(\gamma)}{d \gamma}=\frac{d \rho^{+}(\gamma)}{d \gamma}-\frac{d \rho^{-}(\gamma)}{d \gamma}$

The dislocation storage rate is proportional to the mean free path for dislocations which incorporates the effects of dislocation-dislocation $\left(\Lambda_{\text {pure }}^{-1}\right)$ and solute atom-dislocation interactions, including substitutional $\left(\Lambda_{\text {subs }}^{-1}\right)$ and interstitial solute atoms $\left(\bigwedge_{\text {inter }}^{-1}\right)$ effects:

$\frac{d \rho^{+}(\gamma)}{d \gamma}=\frac{1}{b}\left(\frac{1}{\Lambda_{\text {pure }}}+\frac{1}{\Lambda_{\text {subs }}}+\frac{1}{\Lambda_{\text {inter }}}\right)$

$\Lambda_{\text {pure }}^{-1}=\frac{1}{30}\left(\frac{\mu}{\mu_{0}}\right)^{2} \sqrt{\rho}=K_{1} \sqrt{\rho}, \Lambda_{\text {subs }}^{-1}=\frac{\sum_{i} x_{i}}{16000} \exp \left(-\frac{\Delta G_{\text {sys }}}{R T}\right) \sqrt{\rho}=K_{s s} \sqrt{\rho}$, and $\Lambda_{\text {inter }}^{-1}=\frac{x_{C}^{1 / 3}}{\Lambda_{\text {pure }}^{-1}} . \mu_{0}$ is the shear modulus at $0 \mathrm{~K}, x_{i}$ is the atomic fraction of element $i$, and $\Delta G_{s y s}$ is the free energy of the applied alloy [31].

The dislocation annihilation rate due to dynamic recovery is also expressed as follows:

$\frac{d \rho^{-}(\gamma)}{d \gamma}=f_{D R V} \rho(\gamma)$

where $f_{D R V}$ is the dynamic recovery coefficient. This can be determined by considering the dislocation annihilation energy barrier $\langle\Delta G\rangle$, which contains the formation energy associated with 
the annihilating dislocation segment with the length of $l$, the migration energy of this segment during slip, the contribution of vacancy energy for the dislocation annihilation (especially at high temperatures), the energy term associated with dislocation-substitutional solute atom interaction, and the contribution of statistical entropy that takes account of the number of possible dislocation migration paths [25, 31], as follows:

$$
\begin{aligned}
\langle\Delta G\rangle & =\frac{b}{l}\left(U_{\text {form }}+U_{\text {mig }}+U_{v a c}+U_{S S}-T \Delta S\right) \\
& =\frac{b}{l}\left[\frac{1}{2} \mu b^{2} l+\sigma_{Y} A_{a c t} b^{2} l+\frac{V_{s y s}}{b^{3}} \delta(T)\left(E_{f}+k_{B} T \ln \left(c_{m}\right)\right)+V_{s y s} \frac{1}{b^{3}} \sum_{i} x_{i} \Delta G_{s y s}-k_{B} T \ln (\Omega)\right]
\end{aligned}
$$

where $\sigma_{Y}, A_{a c t}, \delta(T)$, and $E_{f}$ are the yield stress, the activation area associated with dislocation cross slip, a continuum piecewise function in terms of temperature, and the vacancy formation energy respectively. $V_{s y s}=b l^{*} l$ is the annihilating system volume per dislocation, which is proportional to the length of dislocation distortion field over the slip plane, $l^{*}$. This parameter is approximated by $12.5 b . c_{m}=\exp \left(\frac{-\Delta S_{\text {form }}}{R}\right) \exp \left(\frac{-E_{f}}{R T_{m}}\right)$ is the vacancy concentration at melting temperature, where $\Delta S_{\text {form }}$ is the vacancy formation entropy. With regards to the entropy term, dislocation slip and climb are considered to calculate the total number of microstates in the microstructure. In pure materials, $\Omega_{\text {pure }}=\Omega_{\text {dis }}+\Omega_{v-d}=\left(\frac{C}{\langle v\rangle}+\frac{C_{\text {int }}}{\langle v\rangle}\right)^{\mathrm{N}}=\left(\frac{\dot{\varepsilon}_{0}}{\dot{\varepsilon}}+\frac{\vartheta}{\dot{\varepsilon}}\right)^{\mathrm{N}}$ in which $C$, $C_{i n t},\langle v\rangle, \dot{\varepsilon}, \dot{\varepsilon}_{0}$ are the sound speed in the material, the effect of vacancies on dislocation annihilation velocity, dislocation average velocity during annihilation process, axial strain rate, and the maximum possible strain rate in the material, respectively. It should be noted that $C_{i n t}=\frac{\vartheta}{b \rho_{Y}}$ and $\dot{\varepsilon}_{0}=C b \rho_{Y}$ where $\rho_{Y}=\left(\frac{\sigma_{Y}}{0.9 \mu b}\right)^{2}$ is the dislocation density corresponding to the yield point. Moreover, $\mathrm{N}$ is associated with the impingement effect resulting from the overlap of contiguous dislocations strain fields, and $\vartheta=\vartheta_{D} \exp \left(-\frac{E_{m}}{R T}\right)$ is the frequency for vacancy migration in which $\vartheta_{D}=10^{13} \mathrm{~s}^{-1}$ is 
Debye frequency and $E_{m}$ is the vacancy migration energy. Considering the effect of solid solution solute atoms, the above-mentioned equation for the total number of microstates can be extended to $\Omega=\Omega_{\text {pure }} . \Omega_{\text {subs }} . \Omega_{\text {inter }}$. It is found that $\Omega_{\text {subs }}=\left(\Omega_{\text {pure }}\right)^{N_{\text {subs }}}=\left(\frac{\dot{\varepsilon}_{0}+\vartheta}{\dot{\varepsilon}}\right)^{N_{\text {subs }}}$ and $\Omega_{\text {inter }}=$ $\left(\Omega_{\text {pure }}\right)^{x_{c}^{1 / 3}\left(1+N_{\text {subs }}\right)}=\left(\frac{\dot{\varepsilon}_{0}+\vartheta}{\dot{\varepsilon}}\right)^{x_{c}^{1 / 3}\left(1+N_{\text {subs }}\right)}, \quad \quad$ where $N_{\text {subs }}=\sum_{i} x_{i}$

Therefore, $\Omega=\left(\frac{\dot{\varepsilon}_{0}+\vartheta}{\dot{\varepsilon}}\right)^{\mathrm{N}\left(1+x_{c}^{1 / 3}\right)\left(1+N_{\text {subs }}\right)}[25]$.

Dislocation average velocity during annihilation process can also be expressed in Arrhenius form in terms of the annihilation barrier as follows:

$$
\left(\frac{\langle v\rangle}{c+c_{\text {int }}}\right)^{\mathrm{N}\left(1+x_{c}^{1 / 3}\right)}=\left(\frac{\dot{\varepsilon}}{\dot{\varepsilon}_{0}+\vartheta}\right)^{\mathrm{N}\left(1+x_{c}^{1 / 3}\right)}=\exp \left(-\frac{\langle\Delta G\rangle}{k_{B} T}\right)
$$

The average length of annihilating dislocation segments that is obtained by substituting eq. (7) in eq. (8) is applied to determine the dynamic recovery coefficient:

$$
\begin{aligned}
& f_{D R V}=\frac{N_{A}}{w_{a}} \rho_{a} V_{s y s}=\frac{N_{A}}{w_{a}} \rho_{a} b l^{*} l \\
& =\frac{\frac{N_{A}}{w_{a}} \rho_{a} b^{2} l^{*}\left(1+N_{s u b s}\right)\left(1+x_{c}^{1 / 3}\right) k_{B} T \ln \left(\frac{\dot{\varepsilon}_{0}+\vartheta}{\dot{\varepsilon}}\right)^{\mathrm{N}}}{\frac{1}{2} \mu b^{3}+\sigma_{Y} A_{a c t} b^{3}+\frac{l^{*}}{b} \delta(T)\left(E_{f}+k_{B} T \ln \left(c_{m}\right)\right)+\frac{l^{*}}{b} \sum_{i} x_{i} \Delta G_{s y s}-\left(1+x_{c}^{1 / 3}\right) k_{B} T \ln \left(\frac{\dot{\varepsilon}_{0}+\vartheta}{\dot{\varepsilon}}\right)^{\mathrm{N}}}
\end{aligned}
$$

$N_{A}, w_{a}$, and $\rho_{a}$ are the Avogadro's number, the atomic weight of alloy, and the alloy density, respectively [25].

A formulation in the form of Kocks and Mecking equation is employed to predict the dislocation density evolution of microstructural phases during plastic deformation:

$\frac{d \rho_{i}\left(\gamma_{i}\right)}{d \gamma_{i}}=A\left(\frac{K}{b}\right) \sqrt{\rho_{i}\left(\gamma_{i}\right)}-B f_{D R V} \rho_{i}\left(\gamma_{i}\right)$

$K=\left(1+x_{c}^{1 / 3}\right) K_{1}+K_{S S}$. A and $\mathrm{B}$ are structure dependent constants that are considered as 1 for FCC phase (austenite), while they can be calculated for BCC (ferrite/bainite) and BCT phase 
(martensite) through the ratio of the number of slip system times coordination number in these structures to their counterpart in FCC phase [32], as shown in Table 1.

Table 1. Phase dependent coefficients in Kocks and Mecking formulation.

\begin{tabular}{ccccccc}
\hline \hline Phase & Structure & Slip System & $\begin{array}{c}\text { Number of Slip } \\
\text { Systems }\end{array}$ & $\begin{array}{c}\text { Coordination } \\
\text { Number }\end{array}$ & A & B \\
\hline \hline Austenite & FCC & $\{111\}<\overline{1} 10>$ & 12 & 12 & 1 & 1 \\
\hline \multirow{2}{*}{ Ferrite/Bainite } & \multirow{2}{*}{ BCC } & $\{110\}<111>$ & 12 & & & \\
& & $\{123\}<111>$ & 24 & 8 & $\frac{3}{8}$ & $\frac{8}{3}$ \\
& \multirow{2}{*}{ Martensite } & BCT & $\{101\}<\overline{1} 01>$ & 2 & & \\
& & $\{101\}<101>$ & 2 & & $\frac{9}{2}$ & $\frac{2}{9}$ \\
\hline \hline
\end{tabular}

The dislocation density in each individual phase can be obtained as a function of shear strain by solving Eq. 10. According to Taylor relation, shear stress during plastic deformation can also be obtained in terms of shear strain for each microstructural phase as follows:

$\tau_{i}=\alpha M \mu b \sqrt{\rho_{i}\left(\gamma_{i}\right)}$

where $\alpha$ is a constant and $M$ is the Taylor factor [33].

To obtain the stress-strain curve, the iso-work approximation has been taken into account to homogenize the flow behavior in the entire multi-phase structure, which is achieved by assuming that every microstructural phase has the same energy dissipation during deformation [27]:

$\tau_{i} \cdot d \gamma_{i}=\tau_{i+1} \cdot d \gamma_{i+1}$

where $i$ and $i+1$ correspond to different microstructural phases.

The values of the model parameters in the literature have been listed for different microstructural phases in Table 2. 
Table 2. Model parameters in different microstructural phases $[25,27,34]$

\begin{tabular}{|c|c|c|c|c|}
\hline \multirow{2}{*}{ Parameter } & \multicolumn{4}{|c|}{ Phase } \\
\hline & Ferrite & Austenite & Bainite & Martensite \\
\hline$\mu(M P a)$ & \multicolumn{4}{|c|}{75200} \\
\hline$\mu_{0}(M P a)$ & \multicolumn{4}{|c|}{85335} \\
\hline$b(m)$ & \multicolumn{4}{|c|}{$2.5 \mathrm{E}-10$} \\
\hline$D(m)$ & $20 \mathrm{E}-6$ & $15 E-6$ & $\begin{array}{c}\text { artificial neural } \\
\text { network [36] }\end{array}$ & $0.13 \mathrm{E}-6$ \\
\hline$n^{*}$ & 4 & 20 & 4 & 4 \\
\hline$\lambda(m)$ & $1.5 \mathrm{E}-7$ & $1.5 \mathrm{E}-7$ & $1.5 \mathrm{E}-8$ & $5.0 \mathrm{E}-8$ \\
\hline$R(m)$ & $4.5 \mathrm{E}-9$ & $50.0 \mathrm{E}-9$ & $30.0 \mathrm{E}-9$ & $1.0 \mathrm{E}-15$ \\
\hline$\alpha$ & \multicolumn{4}{|c|}{0.25} \\
\hline$M$ & \multicolumn{4}{|c|}{3.06} \\
\hline$\tau_{0}(M P a)$ & \multicolumn{4}{|c|}{18} \\
\hline$\rho_{0}\left(m^{-2}\right)$ & $1 \mathrm{E}+13$ & $1 \mathrm{E}+13$ & $\left(0.7+3.5 w_{C}\right) \times 10^{15}$ & $\begin{array}{c}10^{9.284+\frac{6880}{T_{\text {BIT }}-298}-\frac{1780360}{\left(T_{\text {BIT }}-298\right)^{2}}} \\
{[35]}\end{array}$ \\
\hline $\mathrm{N}$ & 2 & 1 & 2 & 2 \\
\hline$\lambda_{s s}$ & \multicolumn{4}{|c|}{16000} \\
\hline$\delta(T)$ & \multicolumn{4}{|c|}{$\frac{T-T_{0}}{T_{f}-T_{0}}$} \\
\hline$\chi\left(\frac{J}{m^{2}}\right)$ & \multicolumn{4}{|c|}{0.077} \\
\hline$l^{*}$ & \multicolumn{4}{|c|}{$31.25 \mathrm{E}-10$} \\
\hline$E_{\mathrm{f}}(e V)$ & \multicolumn{4}{|c|}{1.6} \\
\hline$E_{\mathrm{m}}(\mathrm{eV})$ & \multicolumn{4}{|c|}{1.28} \\
\hline$\Delta S_{\text {form }}\left(\frac{J}{m o l ~ K}\right)$ & \multicolumn{4}{|c|}{-12.47} \\
\hline$v$ & \multicolumn{4}{|c|}{0.27} \\
\hline$\Delta G_{\text {sys }}\left(\frac{\mathrm{kJ}}{\mathrm{mol}}\right)$ & \multicolumn{4}{|c|}{$16-0.045 T-1.4 * 10^{-5} T^{2}$} \\
\hline$\sigma_{\mathrm{y}}(M P a)$ & 475 & 720 & 475 & 2000 \\
\hline
\end{tabular}




\subsection{Strain-Induced Martensitic Transformation Model}

The martensitic transformation response in TRIP steels during deformation is described by Haidemenopoulos model [26] based on the Olson-Cohen theory for heterogeneous martensitic nucleation. In this model, a composite microstructure of dispersed retained austenite particles in a ferritic matrix is assumed in which martensitic nucleus is formed in austenite phase owing to the dissociation of existing microstructural defects. Generally, there are two types of mechanicallyinduced martensitic nucleation, including stress-assisted nucleation on traditional pre-existing sites (or defects) and strain-induced nucleation on sites produced during plastic deformation. These sites require a sufficient potency for instantaneous nucleation of martensitic phase. The potency of nucleation sites are determined by the number of crystal planes $\left(n_{\text {crys }}\right)$ in the fault formed from their dissociation. Therefore, the sufficient potency corresponding to a critical number of crystal planes $\left(n_{\text {crys }}^{*}\right)$ can be calculated when the formation energy of the $n_{\text {crys }}$-plane fault $\left(\gamma_{f}\left(n_{\text {crys }}\right)\right)$ becomes zero:

$\gamma_{f}\left(n_{\text {crys }}\right)=n_{\text {crys }} \rho\left(\Delta G_{c h}+\mathrm{E}_{\text {str }}+\mathrm{W}_{f}\right)+2 \gamma_{s}$

$\gamma_{f}\left(n_{\text {crys }}^{*}\right)=0 \quad \rightarrow \quad n_{c r y s}^{*}=-\frac{2 \gamma_{s}}{\rho\left(\Delta G_{c h}+\mathrm{E}_{s t r}+\mathrm{W}_{f}\right)}$

where $\rho, \gamma_{s}, \Delta G_{c h}, \mathrm{E}_{s t r}$, and $\mathrm{W}_{f}$ are the atom density of the fault plane, the interfacial energy between fault and matrix, the chemical driving force of martensitic nucleation, the elastic energy at fault/matrix interface, and the frictional work resulting from the movement of fault/matrix interface, respectively.

According to the above-mentioned nucleation mechanisms, the total number of nucleation sites with sufficient potency per unit austenite volume $\left(N_{v}\right)$ is the summation of operational stress-assisted nucleation sites $\left(N_{v}^{\sigma}\right)$ and operational strain-induced nucleation sites $\left(N_{v}^{\varepsilon}\right)$, which are defined as follows:

$N_{v}(\varepsilon)=N_{v}^{\sigma}+N_{v}^{\varepsilon}(\varepsilon)$
$N_{v}^{\sigma}=N_{v}^{\sigma 0} \exp \left(-a_{\sigma} n^{*}\right)$ 
$N_{v}^{\varepsilon}(\varepsilon)=N_{v}^{\varepsilon 0}(\varepsilon) \exp \left(-a_{\varepsilon} n^{*}\right)=N\left(1-\exp \left(-k \varepsilon^{m}\right)\right) \exp \left(-a_{\varepsilon} n^{*}\right)$

where $k$ and $m$ are constants, $N$ is the maximum possible number of strain-induced sites, $\mathrm{a}_{\sigma}$ and $\mathrm{a}_{\varepsilon}$ are the constants in the above exponential functions called shape factors of stress-modified and strain-modified potency distribution, respectively. It is assumed that the nucleation sites formed during plastic deformation possess higher potency compared to the pre-existing nucleation sites, which corresponds to a lower value of $\mathrm{a}_{\varepsilon}$.

Accordingly, the evolution of martensite volume fraction $(f)$ in terms of plastic strain can be written as:

$f(\varepsilon)=1-\exp \left(-v_{p} N_{v}(\varepsilon)\right)$

It is worth noting that the effect of austenite particle size is also taken into account in this model. In Table 3, the parameter values for the applied SIM model have been indicated [26].

Table 3. SIM Model parameters [26]

\begin{tabular}{cc}
\hline \hline Parameter & Value \\
\hline \hline $\mathrm{v}_{\mathrm{p}}\left(\mathrm{m}^{3}\right)$ & $4.18 \mathrm{E}-18$ \\
$\gamma_{\mathrm{s}}\left(\frac{\mathrm{J}}{\mathrm{m}^{2}}\right)$ & 0.15 \\
$\Delta G_{c h}\left(\frac{\mathrm{J}}{m o l}\right)$ & -3285 \\
$\mathrm{E}_{s t r}\left(\frac{J}{m o l}\right)$ & 500 \\
$\mathrm{~W}_{f}\left(\frac{J}{m o l}\right)$ & $1.893 \mathrm{E} 3 * X_{M n}^{2 / 3}+1.310 \mathrm{E} 4 * X_{C}^{2 / 3}$ \\
$\rho\left(\frac{m o l}{m^{2}}\right)$ & $3 \mathrm{E}-5$ \\
$N_{v}^{\sigma 0}\left(m^{-3}\right)$ & $1.5 \mathrm{E} 17$ \\
$N\left(m^{-3}\right)$ & $1.9 \mathrm{E} 19$ \\
$\mathrm{a}_{\sigma}$ & 0.1 \\
$\mathrm{a}_{\varepsilon}$ & 0.03 \\
$k$ & 4.45 \\
$m$ & 46 \\
\hline \hline
\end{tabular}




\subsection{Metropolis-Hastings Algorithm}

Metropolis-Hastings algorithm is one of the MCMC techniques to randomly sample from a multidimensional probability distribution. It is usually applied to obtain the statistical characteristics in the framework of Bayes' theorem for a given set of variables. Since solving the intractable integrals associated with the application of Bayesian framework for model calibration through analytical or numerical approaches is very hard in the high-dimensional cases, MCMC sampling from a joint posterior probability distribution is considered as a good solution [37].

In this approach, experimental data and prior knowledge including the initial values of parameters and their range obtained from literature and/or prior deterministic optimizations are fed to the Matlab MCMC toolbox [38]. In this work, least square optimization techniques have been employed to suggest the beginning point in the parameter space $\left(\theta^{(0)}\right)$ in order to improve the MCMC efficiency for parameter convergence to their plausible mean values, which results in a lower computational cost. Non-informative uniform probability density functions (PDFs) have been also considered as parameter prior distributions since no statistical information has been found for the given model parameters. MCMC toolbox uses random walk in multi-dimensional parameter space to generate $\mathrm{n}$ parameter samples. A new sample or candidate has been drawn from an arbitrary proposal distribution $q$. In our research, an adaptive Gaussian distribution is considered as the proposal density which is centered at the initial or the last accepted parameter with an adaptive variancecovariance. In each generation step, the adaption takes place using the variance-covariance of the previously generated samples based on Haario et al. work [39]. Acceptance/rejection of new sampled candidate is performed by calculating the Metropolis-Hastings ratio:

$$
M H=\frac{\pi\left(\theta^{\text {cand }}\right)}{\pi\left(\theta^{i}\right)} \frac{q\left(\theta^{i} \mid \theta^{\text {cand }}\right)}{q\left(\theta^{\text {cand }} \mid \theta^{i}\right)}=\frac{p\left(\theta^{\text {cand }}\right) p\left(\theta^{\text {cand }} \mid D\right) q\left(\theta^{i} \mid \theta^{\text {cand }}\right)}{p\left(\theta^{i}\right) p\left(\theta^{i} \mid D\right) q\left(\theta^{\text {cand }} \mid \theta^{i}\right)}
$$


where $\frac{\pi\left(\theta^{\text {cand }}\right)}{\pi\left(\theta^{i}\right)}$ is the Metropolis ratio that is the joint probability distribution for the new candidate over the previous one, and $\frac{q\left(\theta^{i} \mid \theta^{\text {cand }}\right)}{q\left(\theta^{\text {cand }} \mid \theta^{i}\right)}$ is the ratio of proposal density in two different directions, i.e., jump from $\theta^{i}$ to $\theta^{\text {cand }}$ and vice versa. The new candidate can be accepted with the minimum probability between the above ratio and 1 . It should be noted that the previous parameter vector is used as the new sample in the case of rejection. At the end of this process, $n$ samples of parameter vectors $\left\{\theta^{(0)}, \ldots, \theta^{(n)}\right\}$ are produced, which show the posterior PDFs of given parameters. It is worth noting that the likelihood function is defined as the difference of the model result at $\theta$ and corresponding data values $(\operatorname{Modelfun}(\theta)-D)$. In this work, the likelihood function is considered as a Gaussian function that is centered at data; its variance (error of data) is regarded as a hyperparameter and updated in the framework of Bayes' theorem during MCMC sampling. More details can be found in Gelman et al. [40]. Flow-chart of this Approach has been shown in Fig.1.

Fig.1. Flow-chart of Metropolis-Hastings method (RNG: Random Number Generation)

In the present work, the models are trained with three different experimental data sequentially and simultaneously to discern how the training sequence can affect the calibration results. In the case of sequential training, the prior PDFs for new training are the posterior PDFs of the previous training. After all the trainings, the mean and standard deviation of the obtained representative samples from parameter posterior PDFs are identified as the plausible mean values and the uncertainty of parameters.

\subsection{Propagation of Uncertainty}

The mean and covariance of generated samples in MCMC toolbox are used to calculate the uncertainty of the model result, which is the uncertainty of deformation stress in terms of plastic strain in our work. This can be performed through propogation of uncertainty as follows: 


$$
\sigma_{S}^{2}=\sum_{i}^{N}\left|\frac{\partial M}{\partial a_{i}}\right|^{2} \sigma_{i}^{2}+\sum_{i}^{N} \sum_{i \neq j}^{N}\left|\frac{\partial M}{\partial a_{i}}\right|\left|\frac{\partial M}{\partial a_{j}}\right| \sigma_{i j}
$$

where $M, a_{i \text { or } j}, \sigma_{i}^{2}$, and $\sigma_{i j}$ are the model function, applied model parameters, parameter variances that is the diagonal elements of variance-covariance matrix, and the covariance of any two parameters that is the off-diagonal elements of variance-covariance matrix, respectively.

The partial derivatives with respect to the parameters have numerically been calculated using the following method $\left(\Delta a_{i}=10^{-5}\right)[41]$ :

$$
\frac{\partial M\left(a_{1}, a_{2}, \ldots, a_{i}, \ldots\right)}{\partial a_{i}} \approx \frac{M\left(a_{1}, a_{2}, \ldots, a_{i}+\frac{\Delta a_{i}}{2}, \ldots\right)-M\left(a_{1}, a_{2}, \ldots, a_{i}-\frac{\Delta a_{i}}{2}, \ldots\right)}{\Delta a_{i}}
$$

\subsection{Applied Experimental Data}

Experimental data reported by Jacques et al. [42] for stress-strain behavior in multi-phase TRIP and dual phase steels has been taken into account for the calibration of the models' parameters. Nominal chemical composition, volume fraction of phases, carbon weight percentage of phases, bainitic isothermal transformation (BIT) temperature, tensile deformation temperature (RT) and strain rate (6.67E-4 s $\left.\mathrm{s}^{-1}\right)$ of these experiments are used as inputs for our models, which are listed in Table 4. It should be noted that inter-critical annealing (IA) temperature and time besides BIT time are not directly among the model inputs, but their effects will be considered through phase volume fractions and carbon contents. For instance, in the case of BIT temperature at $370^{\circ} \mathrm{C}$, prolonged BIT time results in the formation of dual phase ferrite-bainite microstructure, whereas short BIT time of $0.5,1$, and $3 \mathrm{~min}$ for TRIP 4, 5, and 6 causes the formation of multi-phase microstructures, which have austenite and/or martensite besides bainite and ferrite. 
Table 4. Applied Experimental information [41] as model inputs

\begin{tabular}{|c|c|c|c|c|c|c|c|c|c|c|c|c|c|c|c|}
\hline \multirow{2}{*}{ Alloy } & \multicolumn{6}{|c|}{ Chemical Composition (wt\%) } & \multicolumn{4}{|c|}{ Volume Fraction (\%) } & \multicolumn{4}{|c|}{ Carbon Content (wt\%) } & \multirow{2}{*}{$\begin{array}{l}\text { BIT } \\
\left({ }^{\circ} \mathrm{C}\right)\end{array}$} \\
\hline & $\mathrm{C}$ & $\mathrm{Si}$ & $\mathrm{Mn}$ & $\mathrm{Al}$ & $\mathrm{Ni}$ & $\mathrm{Cr}$ & Fer & Aus & Bai & Mar & Fer & Aus & Bai & Mar & \\
\hline TRIP 1 & & & & & & & 75 & 8 & 15 & 0 & 0.014 & 0.93 & 0.3 & 0 & 410 \\
\hline TRIP 2 & 0.13 & 1.5 & 1.42 & 0.027 & 0.02 & 0.013 & 60 & 7.9 & 30 & 0 & 0.01 & 0.85 & 0.2 & 0 & 360 \\
\hline TRIP 3 & & & & & & & 60 & 7.8 & 30 & 0 & 0.01 & 0.97 & 0.16 & 0 & 410 \\
\hline TRIP 4 & & & & & & & 75 & 8.8 & 9 & 7 & 0.02 & 0.61 & 0.3 & 0.97 & \\
\hline TRIP 5 & & & & & & & 75 & 8.1 & 12 & 5 & 0.02 & 0.68 & 0.3 & 1.1 & \\
\hline & 0.16 & 0.38 & 1.3 & 0.03 & 0.027 & 0.019 & & & & & & & & & 370 \\
\hline TRIP 6 & & & & & & & 75 & 6.1 & 19 & 0 & 0.02 & 0.73 & 0.53 & 0 & \\
\hline DP & & & & & & & 75 & 0 & 25 & 0 & 0.02 & 0 & 0.58 & 0 & \\
\hline
\end{tabular}

* Fer: ferrite, Bai: bainite, Aus: retained austenite, Mar: martensite, and DP: Dual Phase

\section{Results and Discussion}

\subsection{Forward Analysis of the Models}

Using the parameter values in Table 2 and 3, stress- strain curves have been estimated in Fig. 2 based on different conditions mentioned in Table 4. These plots show that there are significant discrepancies between model results and experimental data, except for dual phase case. Regardless of inherent uncertainty, these disagreements can be due to three different reasons as mentioned before: 1) the uncertainty in experiments corresponding to the error of tensile test and microstructural characterization, 2) the uncertainty in the models due to their lack of physics, and 3) uncalibrated model parameters.

In order to evaluate the uncertainty of the models, the calibration of the model parameters is a necessary task. Since MCMC approach is an expensive method for model calibration, the most sensitive model parameters are selected using model forward analysis. This has been performed by changing the parameters one by one to identify the sensitivity of model parameters, some of which 
are shown in Fig. 3 for TRIP 4 as examples. In Fig. 3 (a) and (b), high sensitivity of Taylor constant $\alpha$ (alpha) and Peierls stress $\tau_{0}$ (Stress-Fe) is noticeable. It is worth noting that these two parameters mainly affect the initiation of the model response and the curvature remains unchanged. However, the constants in SIM model, i.e., m-SIM and K-SIM, and $n^{*}$ in Eq. 2 for bainite, ferrite and martensite $(\mathrm{nmax}(\mathrm{BFM}))$ change the curvature of the model results without any variation at initial point, as shown in Fig. 3(c-e). In other words, these parameters influence the strain hardening response of the model which is much more significant in the case of m-SIM. The effect of the variation of $n^{*}$ in austenite $(\operatorname{nmax}(\mathrm{A}))$ has also been brought in Fig. 3(f) in order to show one of the insensitive model parameters that has been disregarded for calibrations.

After the forward analysis, the eight most sensitive model parameters have been chosen for calibration, which include four parameters from each model, i.e., microstructural evolution model and SIM model.

Fig.2. (a-g) comparison of experimental data with model results for plastic flow behavior in TRIP (1-6) and DP

Fig.3. Model forward analysis based on data corresponding to TRIP 4 for evaluation of the sensitivity of model parameters

\subsection{Sequential and Simultaneous Calibration of Model Parameters}

The model has been sequentially calibrated for the selected parameters with three different experimental data, including TRIP 2, 1, and 5 in turn. As mentioned in Section 2.3, the parameter posteriors obtained from previous calibration are considered as the parameter prior for next calibration in this approach. The three experimental data referred to are applied all at once during the simultaneous calibration as well. Then, the sequentially/simultaneously calibrated parameters are used to obtain strain-stress curves under other experimental conditions in order to test the validity of the model. In each one of the sequential or simultaneous calibrations, 10000 samples are generated. 
The mean and covariance of the samples can subsequently be introduced as calibrated parameters and their uncertainties, as listed in Table 5. Histograms of parameter posterior PDFs has also been plotted for both calibration methods in Fig. 4 and 5. In the case of sequential calibration (Fig. 4), mSIM, alpha, n-max, and shear modulus $(\mu)$ contain a clear peak in their posterior distribution unlike $\mathrm{a}_{\sigma}$ (a-stress), $\mathrm{a}_{\varepsilon}$ (a-strain), K-SIM, and Stress-Fe whose histograms show multiple peaks suggesting equi-finality problem, which is attributed to the presence of too many parameters for calibration (over-parameterization) and/or the lack of data to constrain the parameter values [43]. Simultaneous calibration also results in similar trends for parameter posterior distributions as shown in Fig. 5; however, the posterior PDFs are more defined for K-SIM and Peierls stress, and the distribution peak of alpha, n-max and shear modulus shift to lower values compared to their counterparts in sequential calibration. The latter is also confirmed by lower mean values for alpha, $\mathrm{n}$-max and shear modulus in Table 5. It should be noted that the significant narrow peak in shear modulus posterior distribution is attributed to the acceptance of lots of candidates between 75200 and $75300 \mathrm{MPa}$ in this case.

Table 5. Applied parameter values and their uncertainties after sequential and simultaneous calibrations compared to initial values before calibrations

\begin{tabular}{|c|c|c|c|c|c|c|c|c|}
\hline \multirow{2}{*}{ Parameters } & \multicolumn{4}{|c|}{4 SIMT Model Parameters } & \multicolumn{4}{|c|}{4 Strain-Stress Model Parameters } \\
\hline & $\mathbf{a}_{\sigma}$ & $a_{\varepsilon}$ & $\mathbf{k}$ & m & $\alpha$ & n-max & $\mu$ & $\sigma_{\mathrm{p}}$ \\
\hline Before Calibration & 0.1 & 0.03 & 46 & 3.45 & 0.25 & 4 & 75200 & 18 \\
\hline $\begin{array}{l}\text { After Sequential } \\
\text { Calibration }\end{array}$ & $\begin{array}{l}0.28 \pm \\
0.125\end{array}$ & $\begin{array}{c}0.056 \pm \\
0.026\end{array}$ & $\begin{array}{l}50.8 \pm \\
16.54\end{array}$ & $\begin{array}{c}1.77 \pm \\
0.51\end{array}$ & $\begin{array}{c}0.256 \pm \\
0.039\end{array}$ & $\begin{array}{c}6.31 \pm \\
1.52\end{array}$ & $\begin{array}{l}76600 \pm \\
2612\end{array}$ & $\begin{array}{c}20.4 \pm \\
5.85\end{array}$ \\
\hline $\begin{array}{c}\text { After Simultaneous } \\
\text { Calibration }\end{array}$ & $\begin{array}{c}0.27 \pm \\
0.13\end{array}$ & $\begin{array}{c}0.051 \pm \\
0.025\end{array}$ & $\begin{array}{c}49.55 \pm \\
15.2\end{array}$ & $\begin{array}{c}1.92 \pm \\
0.74\end{array}$ & $\begin{array}{c}0.176 \pm \\
0.036\end{array}$ & $\begin{array}{c}4.55 \pm \\
1.80\end{array}$ & $\begin{array}{c}75155 \pm \\
1502\end{array}$ & $\begin{array}{l}23.41 \\
\pm 5.20\end{array}$ \\
\hline
\end{tabular}

Fig.4. Histograms of posterior PDFs of applied parameters after sequential calibration

Fig.5. Histograms of posterior PDFs of applied parameters after simultaneous calibration 
The parameter posterior distributions can also reflect the correlation between parameters that are missed in deterministic approaches for parameter calibrations such as least squares. Two dimensional graphs can demonstrate the correlation of pair parameters (their joint probability distributions), some of which is shown in Fig. 6 and 7 for sequential and simultaneous calibration, respectively. The Pearson correlation coefficient is applied to determine the linear correlation between each two model parameters using the variance-covariance matrix obtained from MCMC sampling as follows [44]:

$$
\rho_{X, Y}=\operatorname{Corr}(X, Y)=\frac{\operatorname{Cov}(X, Y)}{\sigma_{X} \sigma_{Y}}
$$

This can be between -1 and 1 . The maximum and minimum values correspond to perfect correlations and zero means no correlation between parameters. Positive or negative signs indicate increasing or decreasing change of one parameter by the variation of the other one. It is worth noting that the slope of the relationships in the graphs is nothing to do with Pearson correlation. In fact, the correlation depends on how linear the relationship is. The correlation coefficients are calculated for each two model parameters in both of the calibration cases, as observed in Table 6 and 7.

Table 6. Pearson correlation coefficients between model parameters in the case of sequential calibration

\begin{tabular}{c||cccccccc}
\hline \hline & $\mathbf{a}_{\sigma}$ & $\mathbf{a}_{\boldsymbol{\varepsilon}}$ & $\mathbf{k}$ & $\mathbf{m}$ & $\boldsymbol{\alpha}$ & $\mathbf{n}-\mathbf{m a x}$ & $\boldsymbol{\mu}$ & $\sigma_{\mathfrak{p}}$ \\
\hline \hline $\mathbf{a}_{\sigma}$ & 1 & 0.11 & -0.04 & -0.06 & -0.11 & -0.03 & 0.01 & 0.14 \\
$\mathbf{a}_{\boldsymbol{\varepsilon}}$ & 0.11 & 1 & 0.035 & -0.14 & -0.08 & 0.08 & 0.095 & 0.01 \\
$\mathbf{k}$ & -0.04 & 0.035 & 1 & 0.23 & 0.03 & -0.055 & 0.07 & -0.03 \\
$\mathbf{m}$ & -0.06 & -0.14 & 0.23 & 1 & 0.52 & -0.12 & -0.15 & 0.04 \\
$\boldsymbol{\alpha}$ & -0.11 & -0.08 & 0.03 & 0.52 & 1 & -0.39 & -0.40 & -0.57 \\
$\sigma_{\mathbf{p}}$ & 0.14 & 0.01 & -0.03 & 0.04 & -0.57 & 1 & 0.01 & 0.08 \\
$\boldsymbol{\mu}$ & 0.01 & 0.095 & 0.07 & -0.15 & -0.40 & 0.11 & 1 & 0.01 \\
$\mathbf{n}-\mathbf{m a x}$ & -0.03 & 0.08 & -0.55 & -0.12 & -0.39 & 0.08 & 0.11 & 1 \\
\hline \hline
\end{tabular}


Table 7. Pearson correlation coefficients between model parameters in the case of simultaneous calibration

\begin{tabular}{c||cccccccc}
\hline \hline & $\mathrm{a}_{\sigma}$ & $\mathrm{a}_{\boldsymbol{\varepsilon}}$ & $\mathbf{k}$ & $\mathbf{m}$ & $\boldsymbol{\alpha}$ & $\mathrm{n}-\mathrm{max}$ & $\boldsymbol{\mu}$ & $\sigma_{\mathrm{p}}$ \\
\hline \hline $\mathbf{a}_{\sigma}$ & 1 & -0.04 & 0.002 & -0.115 & -0.05 & 0.025 & 0.075 & -0.02 \\
$\mathbf{a}_{\boldsymbol{\varepsilon}}$ & -0.04 & 1 & 0.09 & -0.02 & 0.06 & -0.006 & 0.04 & -0.06 \\
$\mathbf{k}$ & 0.002 & 0.09 & 1 & 0.05 & -0.015 & 0.025 & 0.09 & 0.0002 \\
$\mathbf{m}$ & -0.115 & -0.02 & 0.05 & 1 & 0.27 & 0.09 & -0.001 & -0.09 \\
$\boldsymbol{\alpha}$ & -0.05 & 0.06 & -0.015 & 0.27 & 1 & -0.685 & -0.13 & -0.495 \\
$\sigma_{\mathbf{p}}$ & -0.02 & -0.06 & 0.0002 & -0.09 & -0.495 & 1 & 0.085 & -0.003 \\
$\boldsymbol{\mu}$ & 0.075 & 0.04 & 0.09 & -0.001 & -0.13 & -0.05 & 1 & 0.085 \\
$\mathbf{n}-\mathbf{m a x}$ & 0.025 & -0.006 & 0.025 & 0.09 & -0.685 & -0.003 & -0.05 & 1 \\
\hline \hline
\end{tabular}

In both methods of calibration, there are no/low correlation coefficients between applied parameters, except for alpha, that show some correlations with m-SIM, Peierls stress, and n-max; although, some correlations are also observable between alpha and shear modulus as well as m-SIM and K-SIM in the case of sequential calibration. These can be also evaluated through the correlation graphs in Fig. 6 and 7. In these graphs, red regions indicate the convergence regions of the generated MCMC samples that include the plausible optimal values for the model parameters. The density of samples in 2D parameter spaces decreases from red to blue regions, so the dispersion of points and their linearity in each case can be visually analyzed and verified with the correlation coefficients obtained by Eq. 23. In fact, more scattering of sample points and less linearity (corresponding to a wider ellipsoid and more circular shape) lead to lower correlation coefficient. Another important feature of these graphs is that the equifinality problem is easily distinguishable. For instance, several density peaks are noticeable in Fig. 6(a) and (d). However, this feature cannot be seen in Fig. 7(a) and (d) anymore, which suggests less equifinality problem and more defined posterior PDFs in the case of simultaneous calibration. 
Fig.6. 2D correlation graphs with normalized color bars for some of pair model parameters in the case of sequential calibration

Fig.7. 2D correlation graphs with normalized color bars for some of pair model parameters in the case of simultaneous calibration.

Based on the information in Table 5 about the calibrated parameter values and their uncertainties and considering the propagation of uncertainty, stress-strain curves and their uncertainty bands after sequential and simultaneous calibration have been demonstrated for all experimental conditions in Fig. 8 and 9. The black lines correspond to the plausible mean parameter values obtained after calibrations, and the blue and (blue + green) shaded areas are related to $68 \%$ and $95 \%$ Bayesian confidence intervals of the model predictions, respectively, i.e., $\sigma(\varepsilon) \pm \sigma_{s}(\varepsilon)$ and $\sigma(\varepsilon) \pm 2 \sigma_{s}(\varepsilon)$ in which $\sigma_{s}$ can be determined by Eq. 21 at any strain. According to these plots, it can be mentioned that the model gives fairly close results to experimental data in the cases of TRIP 1, 5, 6, and 7 after sequential calibration, and 1,3,6, and 7 after simultaneous calibration. The outcomes of the model imply a requirement for improvement of the model; although the discrepancies between model and experiments cannot only be attributed to the model deficiency and the effect of experimental errors should also be considered. Therefore, the volume fractions of microstructural phases are estimated in section 3.3 due to high uncertainty in their experimental measurement

Fig.8. Model prediction of strain-stress curves and their uncertainty bands after sequential calibration in different experimental conditions: a-g) TRIP (1-6) and DP, BCI: Bayesian Confidence Interval

Fig.9. Model prediction of strain-stress curves and their uncertainty bands after simultaneous calibration in different experimental conditions: a-g) TRIP (1-6) and DP, BCI: Bayesian Confidence Interval 


\subsection{Estimation of Phase Volume Fractions Using Sequentially Calibrated Parameters}

Generally, the determination of phase volume fractions in steels using image analysis is a hard task and coupled with a lot of uncertainties, especially for ferrite, bainite, and martensite which possess similar crystal structures but different dislocation densities. Therefore, the disagreement between model results and experimental data can be partly related to the experimental uncertainty of phase volume fractions. Assuming the uncertainty of the model is zero and the model is physically correct and complete, the sequentially calibrated parameters have selected for the model to estimate the phase volume fractions by our MCMC approach. Similar to the calibrated parameters in section 3.2, these values and their uncertainties (listed in Table 8) can be applied to plot stress-strain curves and their uncertainty bands, as shown in Fig. 10. It can be observed that the correction of phase volume fractions either results in a very good agreement between the model prediction and corresponding experimental data or at least brings the experimental data to $95 \%$ Bayesian confidence intervals of the model predictions. These estimations can similarly be performed using simultaneously calibrated parameters.

Table 8. MCMC estimation of the phase volume fractions using sequentially calibrated parameters

\begin{tabular}{c|cccc}
\hline \multirow{2}{*}{ Alloy } & \multicolumn{4}{c}{ Volume Fraction (\%) } \\
\cline { 2 - 5 } & Ferrrite & Bainite & Austenite & Martensite \\
\hline \hline TRIP 1 & $74.8 \pm 0.62$ & $3.6 \pm 0.63$ & $10.5 \pm 0.54$ & $11.1 \pm 1.51$ \\
TRIP 2 & $76.6 \pm 2.24$ & $6.5 \pm 1.06$ & $16.9 \pm 1.82$ & 0.0 \\
TRIP 3 & $73.8 \pm 0.26$ & $18.3 \pm 0.35$ & $1.2 \pm 0.44$ & $6.7 \pm 0.58$ \\
TRIP 4 & $75.0 \pm 5.43$ & $4.6 \pm 2.18$ & $20.4 \pm 2.98$ & 0.0 \\
TRIP 5 & $75.6 \pm 5.56$ & $5.8 \pm 2.22$ & $18.6 \pm 2.62$ & 0.0 \\
TRIP 6 & $75.9 \pm 4.06$ & $11.3 \pm 1.97$ & $10.9 \pm 1.78$ & $1.9 \pm 0.58$ \\
DP & $80.0 \pm 1.96$ & $20.0 \pm 0.28$ & 0.0 & 0.0 \\
\hline \hline
\end{tabular}


Fig.10. Model prediction of strain-stress curves and their uncertainty bands after the estimation of phase volume fractions using sequentially calibrated parameters in different experimental conditions: a-g) TRIP (1-6) and DP, BCI: Bayesian Confidence Interval

\subsection{Accuracy Evaluation of the MCMC Calibration Using Generated Synthetic Data}

Regarding the disagreement between model and experimental results, some can state that the uncertainty may result from the inaccuracy of the calibration approach. In order to prove that our approach is reliable for model calibration, the model parameters are simultaneously recalibrated using three synthetic datasets for TRIP 1,2, and 3, which are generated from the model with simultaneously calibrated parameters in Section 3.2 and experimental conditions in Table 4. In fact, the reproducibility of the calibration method is examined by synthetic datasets. As observed in Fig. 11(a), the model with initial parameter values in table 2 and 3 leads to the stress-strain results being very far from the synthetic datasets. However, excellent fits between model results and the synthetic data with very low uncertainties in Fig. 2(b) show that simultaneous recalibration delivers the same parameters used for dataset generations. This confirms a high reproducibility of the introduced MCMC approach. The same scenario is believed to happen in the case of considering sequentially calibrated parameters to generate datasets. This means any discrepancy between model and experimental results can only be attributed to the uncertainty of model and/or experiments, not the applied calibration approach.

Fig.11. Model prediction of strain-stress curves a) before and b) after simultaneous recalibration with synthetic datasets generated using simultaneously calibrated parameters and experimental conditions corresponding to TRIP 1,2 , and 3

\section{Conclusion}

In this work, plastic flow behavior of TRIP steels is predicted through a novel physical-based model that considers different hardening mechanisms, especially dislocation strengthening grains based on Rivera's work, which are considered for each microstructural phase and then homogenized for the 
entire microstructure by an iso-work approximation. Strain-induced martensitic transformation is also incorporated in the model through Haidemenopoulos approach.

The major point of current work is the introduction of Bayesian calibration based on MetropolisHastings MCMC using the above-mentioned models as case studies. After finding eight sensitive parameters through forward analysis of models, two different techniques, i.e., sequential and simultaneous experimental training have been applied to calibrate these parameters. Obtained posterior PDFs and 2D correlation graphs from both calibration methods show a clear peak for mSIM, alpha, n-max, and shear modulus although the distribution peak of alpha, n-max and shear modulus are lower in the case of simultaneous calibration. For the other four parameters, sequential calibration leads to multiple peak or equifinality problems, which is less distinguishable in the results of simultaneous calibration. It should be noted that simultaneous calibration produces a more defined shape of parameter posterior distribution compared to sequential calibration. In both calibration approaches, some of the stress-strain curves resulting from the models with calibrated parameters show disagreements with experimental data; however, the results are better than ones obtained from uncalibrated models overall.

Considering high experimental uncertainties in determination of phase volume fractions and assuming a high accuracy in physics of the models, Bayesian estimation of volume fractions using the calibrated model causes the stress-strain predictions to either fit or be close to experimental results in a way that all data points at least fall in 95\% Bayesian confidence intervals. In order to prove that the uncertainties result from the models and/or experiments and not from our calibration approaches, the models have simultaneously been recalibrated with synthetic datasets generated from the models with simultaneously calibrated parameters. Excellent agreement between model 
results and synthetic datasets after recalibration imply a high reproducibility of the applied MCMC method.

To close the present discussion, we would like to note that while the present work is rather specific in the system under study (TRIP steels) and the selected model (irreversible-thermodynamics of dislocation evolution + iso-work approximation + SIM), the framework presented here is highly generalizable to many other computational materials science problems that require rigorous uncertainty quantification (UQ). In fact, we believe that current emphasis on the use of computational approaches to accelerate the development/design of materials require UQ in order to provide a measure of robustness to the results of materials simulations. Moreover, UQ can also be used to assess the uncertainty in experimental observations and to determine parameters difficult to observe experimentally. While Bayesian methods have been amply used in other fields, they-with some notable exceptions - have not been widely used in computational materials science but their increased use is warranted by the needs of the field.

\section{Acknowledgements}

The authors would like to appreciate Pedro Rivera (Cambridge), Zenon Medina-Cetina (Texas A\&M University) and Shengyen Li (NIST) for their unfailing discussions. This work was supported by the National Science Foundation (NSF) [CMMI-0900187, 095398, and 1534534 (DMREF)].

\section{References}

[1] W.J. Dan, W.G. Zhang, S.H. Li, Z.Q. Lin, A model for strain-induced martensitic transformation of TRIP steel with strain rate, Comp. Mater. Sci. 40 (2007) 101-107.

[2] A. Grajcar, Hot-working in the $\gamma+\alpha$ region of TRIP-aided micro-alloyed steel, Arch. Mater. Sci. Eng. 28 (2007) 743-750. 
[3] B.C. De Cooman, Structure-properties relationship in TRIP steels containing carbide-free bainite, Curr. Opin. Solid St. M. 8 (2004) 285-303.

[4] H.N. Han, C.S. Oh, G. Kim, O. Kwon, Design method for TRIP-aided multiphase steel based on a microstructure-based modelling for transformation-induced plasticity and mechanically induced martensitic transformation, Mater. Sci. Eng. A 499 (2009) 462-468.

[5] E. Girault, P. Jacques, P. Harlet, K. Mols, J. Van Humbeeck, E. Aernoudt, F. Delannay, Metallographic Methods for Revealing the Multiphase Microstructure of TRIP-Assisted Steels, Mater. Charact. 40 (1998) 111-118.

[6] N.H. Van Dijk, A.M. Butt, L. Zhao, J. Sietsma, S.E. Offerman, J.P. Wright, S. Van der Zwaag, Thermal stability of retained austenite in TRIP steels studied by synchrotron X-ray diffraction during cooling, Acta Mater. 53 (2005) 5439-5447.

[7] O. Grässel, L. Krüger, G. Frommeyer, L.W. Meyer, High strength Fe-Mn-(Al, Si) TRIP/TWIP steels development -properties -application, Int. J. Plasticity 16 (2000) 1391-1409.

[8] C. Herrera, D. Ponge, D. Raabe, Design of a novel Mn-based 1 GPa duplex stainless TRIP steel with $60 \%$ ductility by a reduction of austenite stability, Acta Mater. 59 (2011) 4653-4664.

[9] O. Matsumura, Y. Sakuma, H. Takechi, Enhancement of Elongation by Retained Intercritical Annealed 0.4C-1.5Si-0.8Mn Austenite in Steel, Trans. ISIJ 27 (1987) 570-579.

[10] S. Oliver, T.B. Jones, G. Fourlaris, Dual phase versus TRIP strip steels: comparison of dynamic properties for automotive crash performance, Mater. Sci. Technol. 23 (2007) 423-431.

[11] F. Lani, Q. Furnémont, T. Van Rompaey, F. Delannay, P.J. Jacques, T. Pardoen, Multiscale mechanics of TRIP-assisted multiphase steels: II. Micromechanical modelling, Acta Mater. 55 (2007) 3695-3705. 
[12] J.Y. Liu, H. Lu, J.M. Chen, J.F. Jullien, T. Wu, Simulation of mechanical behavior of multiphase TRIP steel taking account of transformation-induced plasticity, Comp. Mater. Sci.43 (2008) 646-654.

[13] J. Bouquerel, K. Verbeken, B.C. De Cooman, Microstructure-based model for the static mechanical behavior of multiphase steels, Acta Mater. 54 (2006) 1443-1456.

[14] A. Perlade, O. Bouaziz, Q. Furnémont, A physically based model for TRIP-aided carbon steels behavior, Mater. Sci. Eng. A 356 (2003) 145-152.

[15] L. Delannay, P. Jacques, T. Pardoen, Modelling of the plastic flow of trip-aided multiphase steel based on an incremental mean-field approach, Int. J. Solids Struct. 45 (2008) 1825-1843. [16] V.F. Zackay, E.R. Parker, D. Fahr, The Enhancement of Ductility on High-Strength Steels [J], T. Am. Soc. Metal. 60 (1967) 252-259.

[17] G.B. Olson, M. Cohen, Kinetics of Strain-Induced Martensitic Nucleation, Metal. Trans. A 6A (1975) 791-795.

[18] G.B. Olson, M. Azrin, Transformation Behavior of TRIP Steels, Metal. Trans. A 9 (1978), 713721.

[19] Y. Tomita, T. Iwamoto, Computational prediction of deformation behavior of TRIP steels under cyclic loading, Int. J. Mech. Sci. 43 (2001) 2017-2034.

[20] S. Thibaud, N. Boudeau, J.C. Gelin, TRIP steel: Plastic behavior modelling and influence on functional behavior, J. Mater. Process. Tech. 177 (2006) 433-438.

[21] H.J. Choi, D.L. McDowell, J.K. Allen, F. Mistree, An inductive design exploration method for hierarchical systems design under uncertainty, Eng. Optimiz. 40 (2008) 287-307.

[22] M. Gallagher, J. Doherty, Parameter estimation and uncertainty analysis for a watershed model, Environ. Modell. Softw. 22 (2007) 1000-1020. 
[23] A. Kanso, G. Chebbo, B. Tassin, Application of MCMC-GSA model calibration method to urban runoff quality modeling, Reliab. Eng. Syst. Safe. 91 (2006) 1398-1405.

[24] W.J. Browne, D. Draper, A comparison of Bayesian and likelihood-based methods for fitting multilevel methods, Bayesian Anal. 1 (2006) 473-514.

[25] E.I. Galindo-Nava, A. Perlade, P.E.J. Rivera-Díaz-del-Castillo, A thermostatistical theory for solid solution effects in the hot deformation of alloys: an application to low-alloy steels, Model. Simul. Mater. Sc. 22 (2014) 1-34.

[26] G.N. Haidemenopoulos, N. Aravas, I. Bellas, Kinetics of strain-induced transformation of dispersed austenite in low-alloy TRIP steels, Mater. Sci. Eng. A 615 (2014) 416-423.

[27] P.E.J. Rivera-Díaz-del-Castillo, K. Hayashi, E.I. Galindo-Nava, Computational design of nanostructured steels employing irreversible thermodynamics, Mater. Sci. Technol. 29 (2013) 12061211.

[28] K.J. Irvine, T. Gladman, F.B. Pickering, The strength of austenitic stainless steels, J. Iron Steel Inst. 207 (1969) 1017-1028.

[29] R.M. Rodriguez, I. Gutierrez, Unified formulation to predict the tensile curves of steels with different microstructures, Mater. Sci. Forum 426 (2003) 4525-4530.

[30] M. Azuma, N. Fujita, M. Takahashi, T. Senuma, D. Quidort, T. Lung, "Modelling upper and lower bainite trasformation in steels, ISIJ Int. 45 (2005) 221-228.

[31] U.F. Kocks, H. Mecking, Physics and phenomenology of strain hardening: the FCC case, Prog. Mater. Sci. 48 (2003) 171-273.

[32] E.I. Galindo-Nava, P.E.J. Rivera-Díaz-del-Castillo, Modelling plastic deformation in BCC metals: Dynamic recovery and cell formation effects, Mater. Sci. Eng. A 558 (2012) 641-648. [33] E.I. Galindo-Nava, P.E.J. Rivera-Díaz-del-Castillo, Thermostatistical modelling of hot deformation in FCC metals, Int. J. Plasticity 47 (2013) 202-221. 
[34] S. Li, P. Honarmandi, R. Arróyave, P.E.J. Rivera-Diaz-del-Castillo, Describing the deformation behavior of TRIP and dual phase steels employing an irreversible thermodynamics formulation, Mater. Sci. Technol. 31 (2015) 1658-1663.

[35] C.H. Young, H. Bhadeshia, Strength of mixtures of bainite and martensite, Mater. Sci. Technol. 10 (1994) 209-214.

[36] S. Singh, H. Bhadeshia, Estimation of bainite plate-thickness in low-alloy steels, Mater. Sci. Eng. A 245 (1998) 72-79.

[37] S.M. Lynch, Introduction to applied Bayesian statistics and estimation for social scientists, Springer Science and Business Media, New York, 2007, 1-6.

[38] M. Laine. (2013, August 27). MCMC toolbox for Matlab. Retrieved from http://helios.fmi.fi/ lainema/mcmc/.

[39] H. Haario, E. Saksman, J. Tamminen, An adaptive metropolis algorithm, Bernoulli 7 (2001) 223-242.

[40] A. Gelman, J.B. Carlin, H.S. Stern, D.B Rubin, Bayesian Data Analysis, Chapman \& Hall/CRC Boca Raton, Florida, 2014, 50-51.

[41] J. Tellinghuisen, Statistical Error Propagation, J. Phys. Chem. A 105 (2001) 3917-3921. [42] P. Jacques, Q. Furnémont, A. Mertens, F. Delannay, On the sources of work hardening in multiphase steels assisted by transformation-induced plasticity, Philos. Mag. 81 (2001) 1789-1812. [43] X. Mo, F. Pappenberger, K. Beven, S. Liu, A. De Roo, Z. Lin, Parameter conditioning and prediction uncertainties of the LISFLOOD-WB distributed hydrological model, Hydrolog. Sci. J. 51 (2006) 45-65.

[44] D.J. Battle, P. Gerstoft, W.S. Hodgkiss, W.A. Kuperman, P.L. Nielsen, Bayesian model selection applied to self-noise geoacoustic inversion, J. Acoust. Soc. Am. 116 (2004) 2043-2056. 


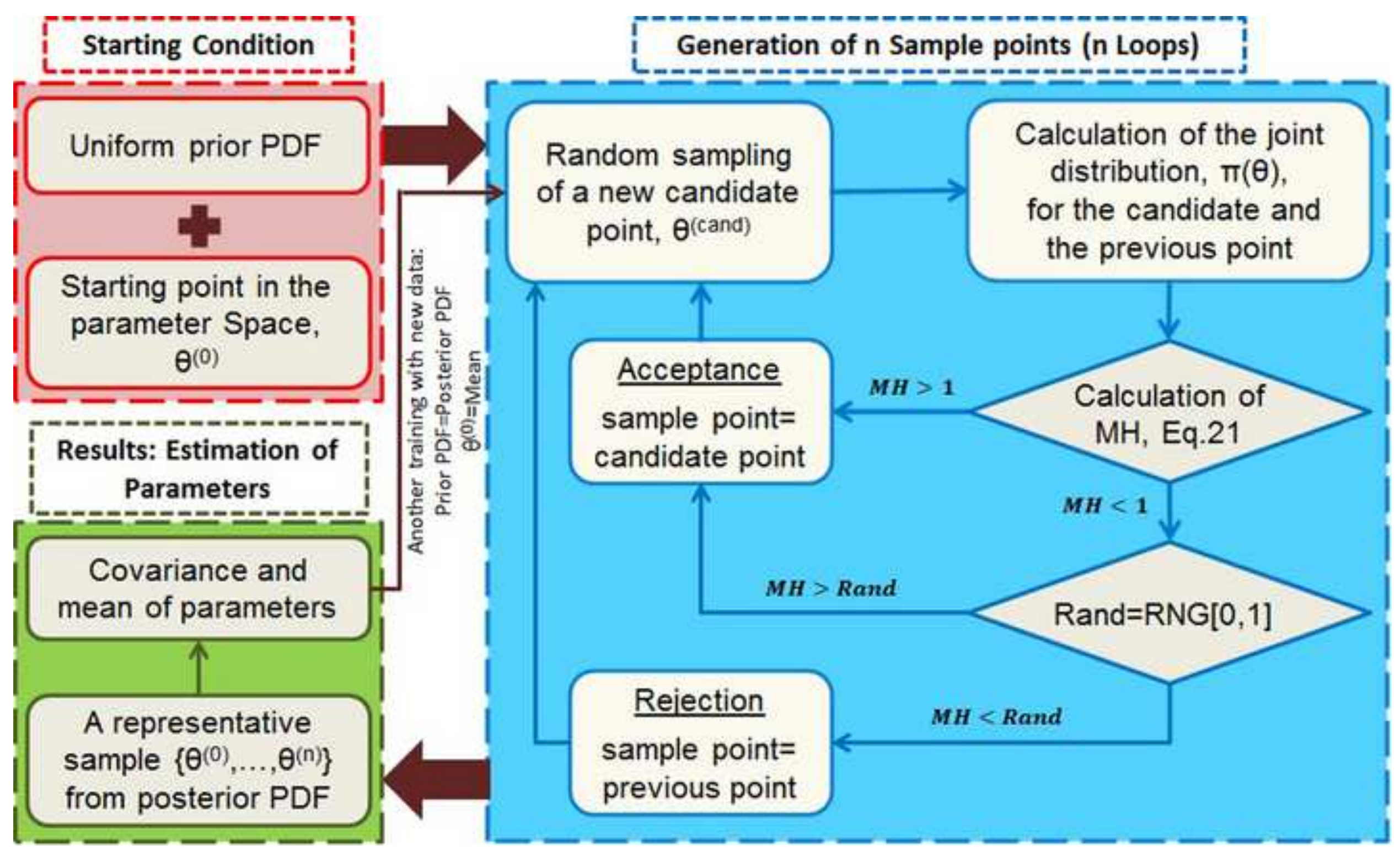




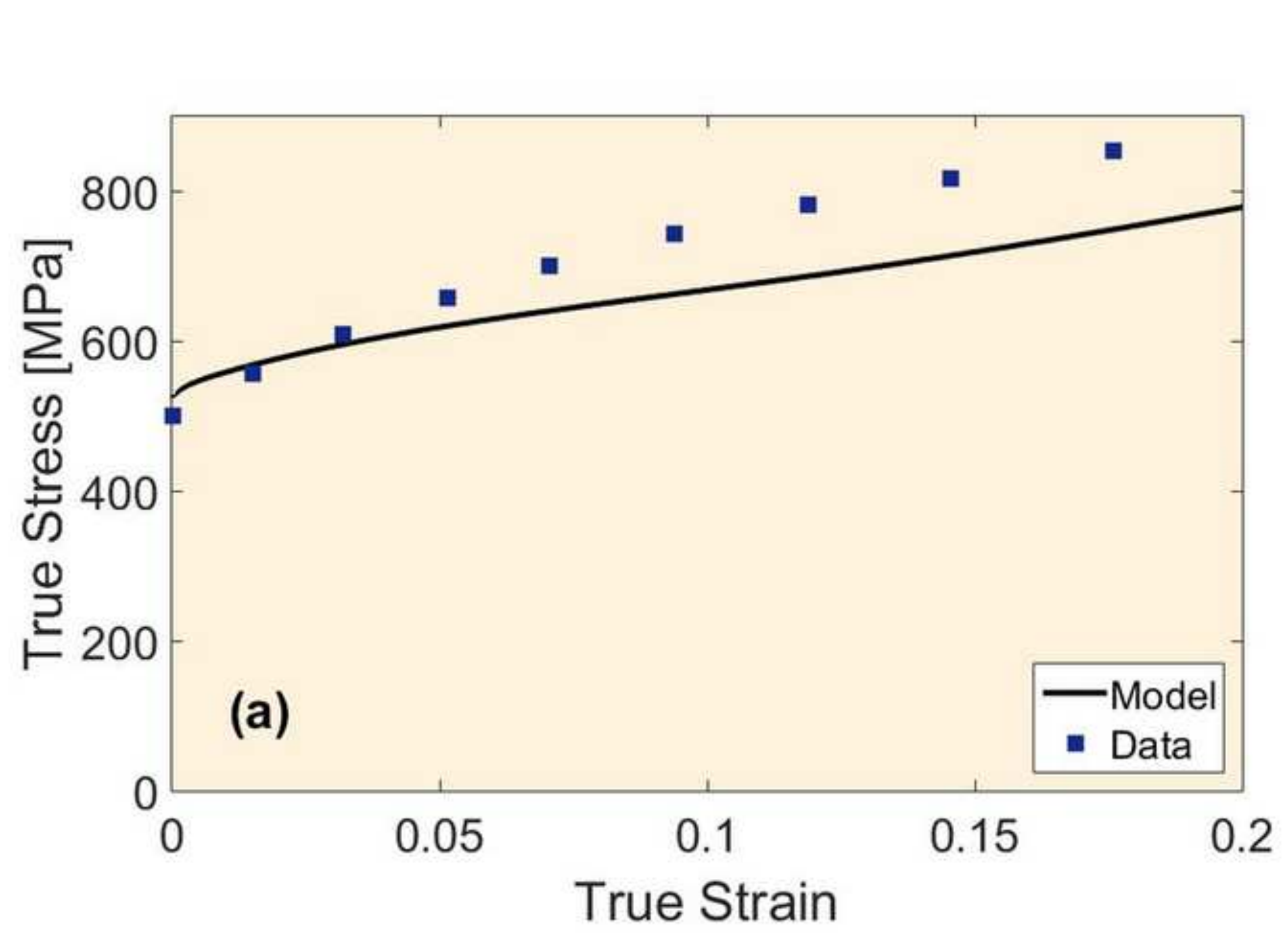

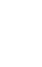




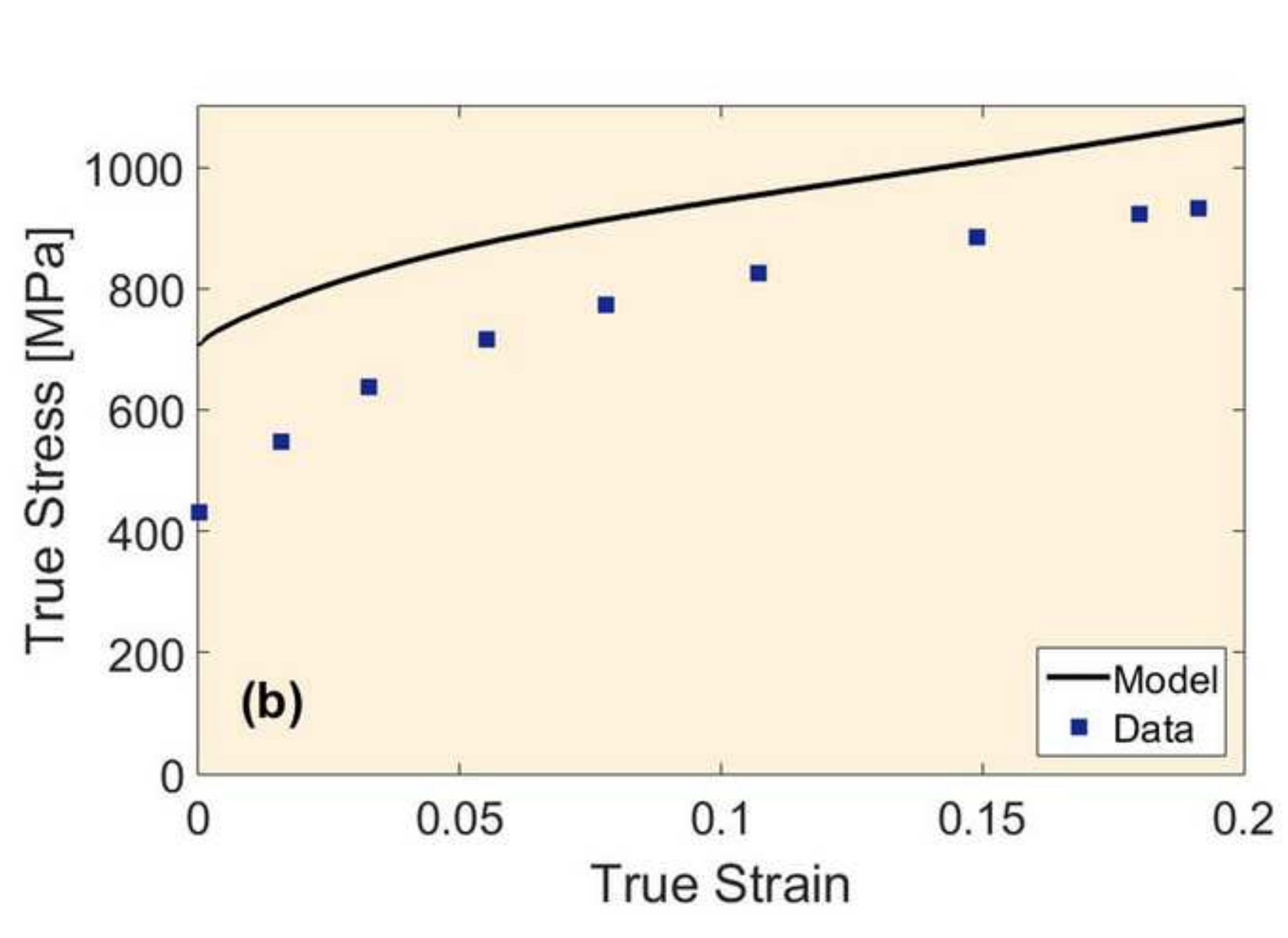

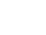

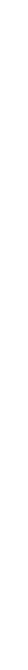

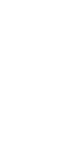

\section{True Strain}

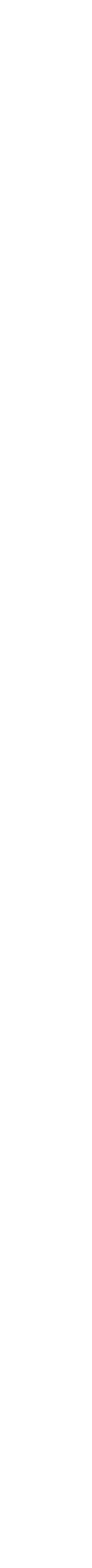

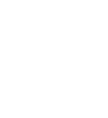

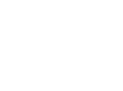

$$
\begin{aligned}
& 0.05 \\
& \text {. }
\end{aligned}
$$

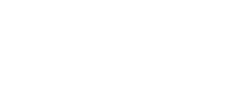

.

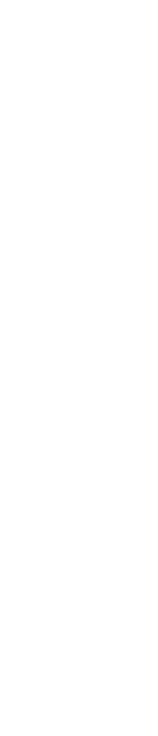

更




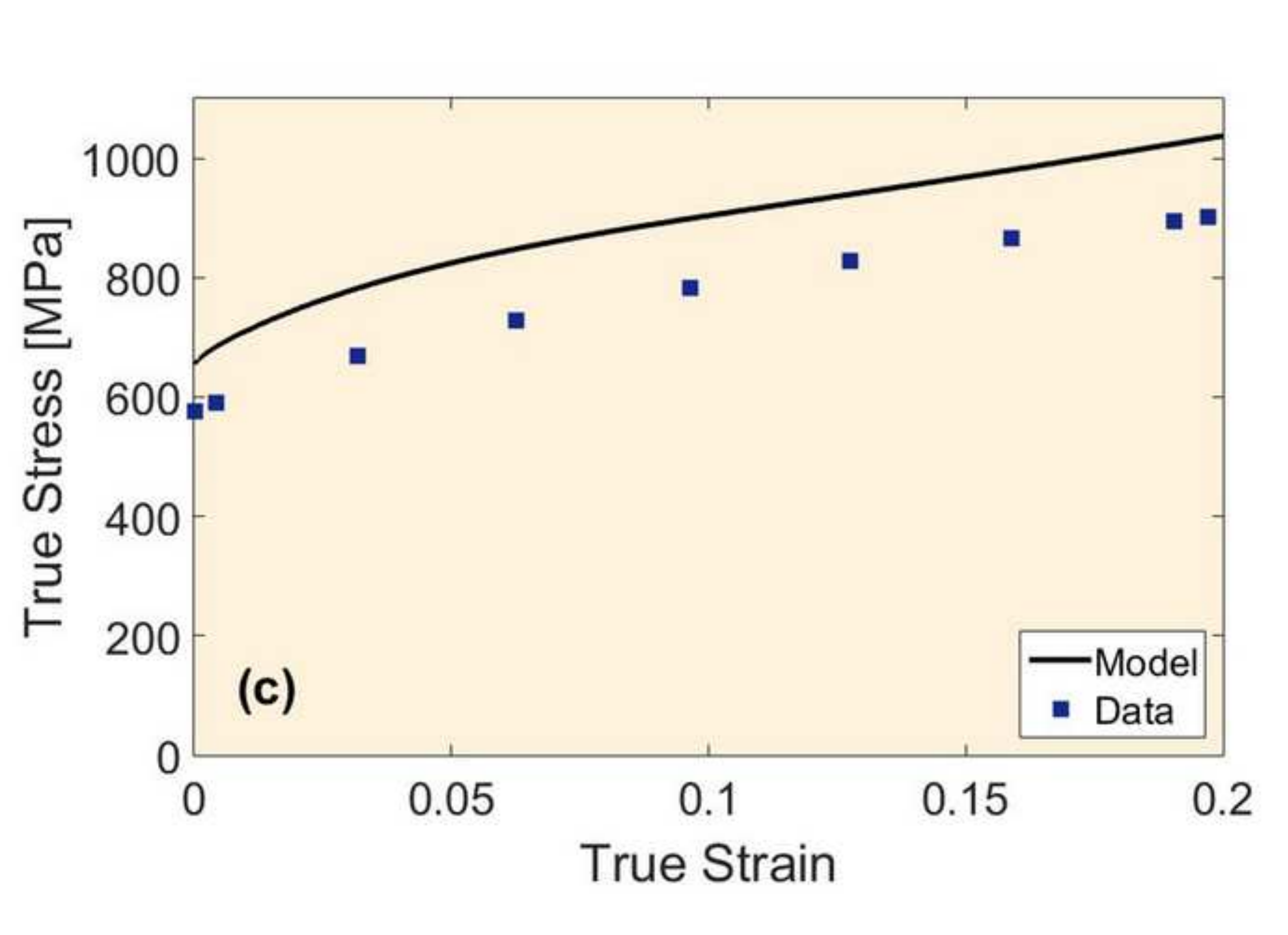

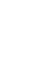

\section{True Strain}

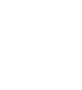

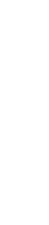

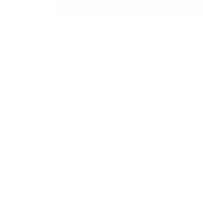

.
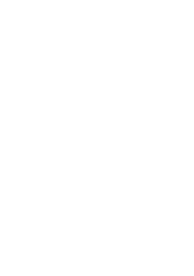


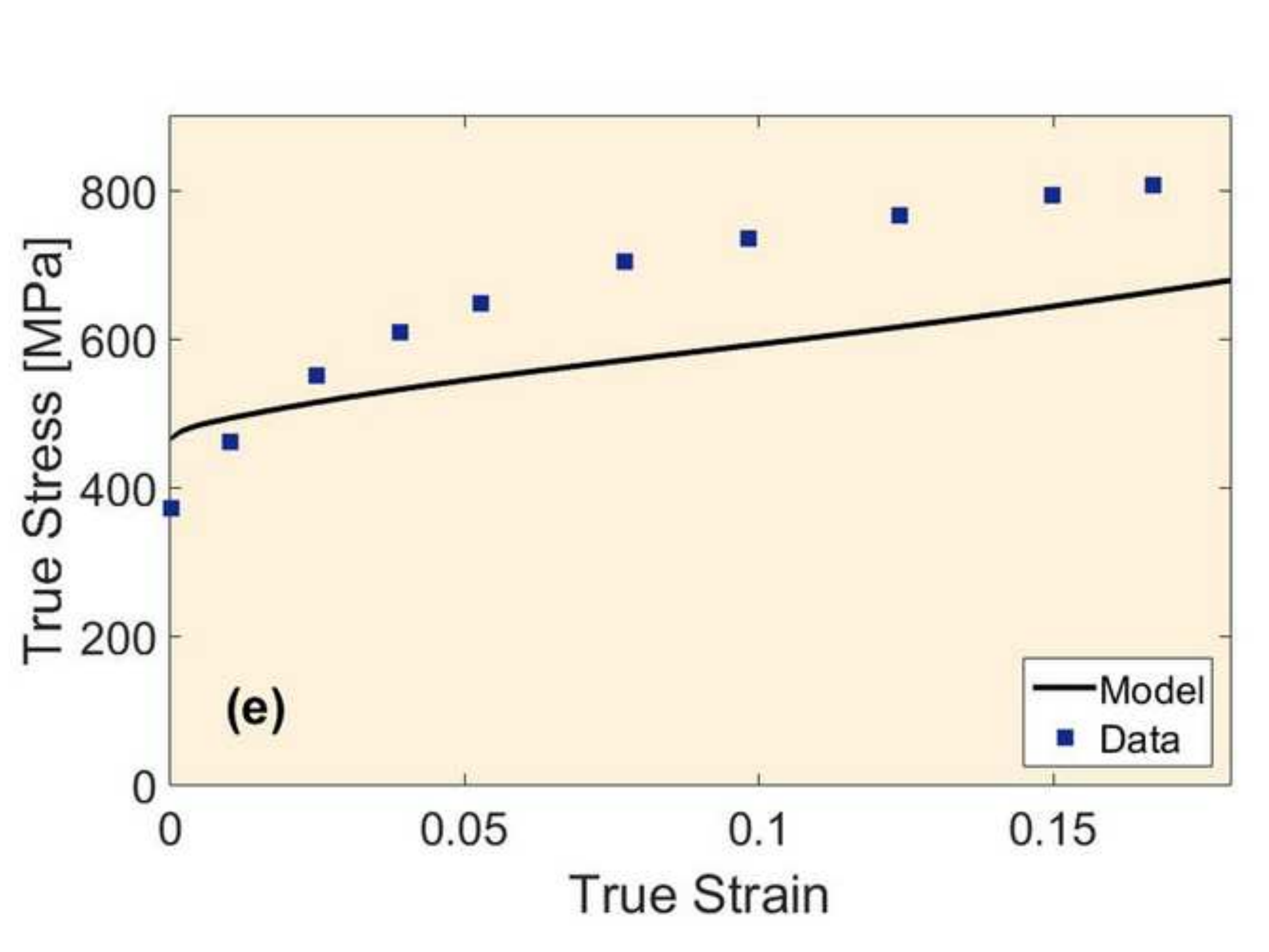

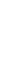

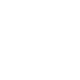

\title{
True Strain
}

(n)




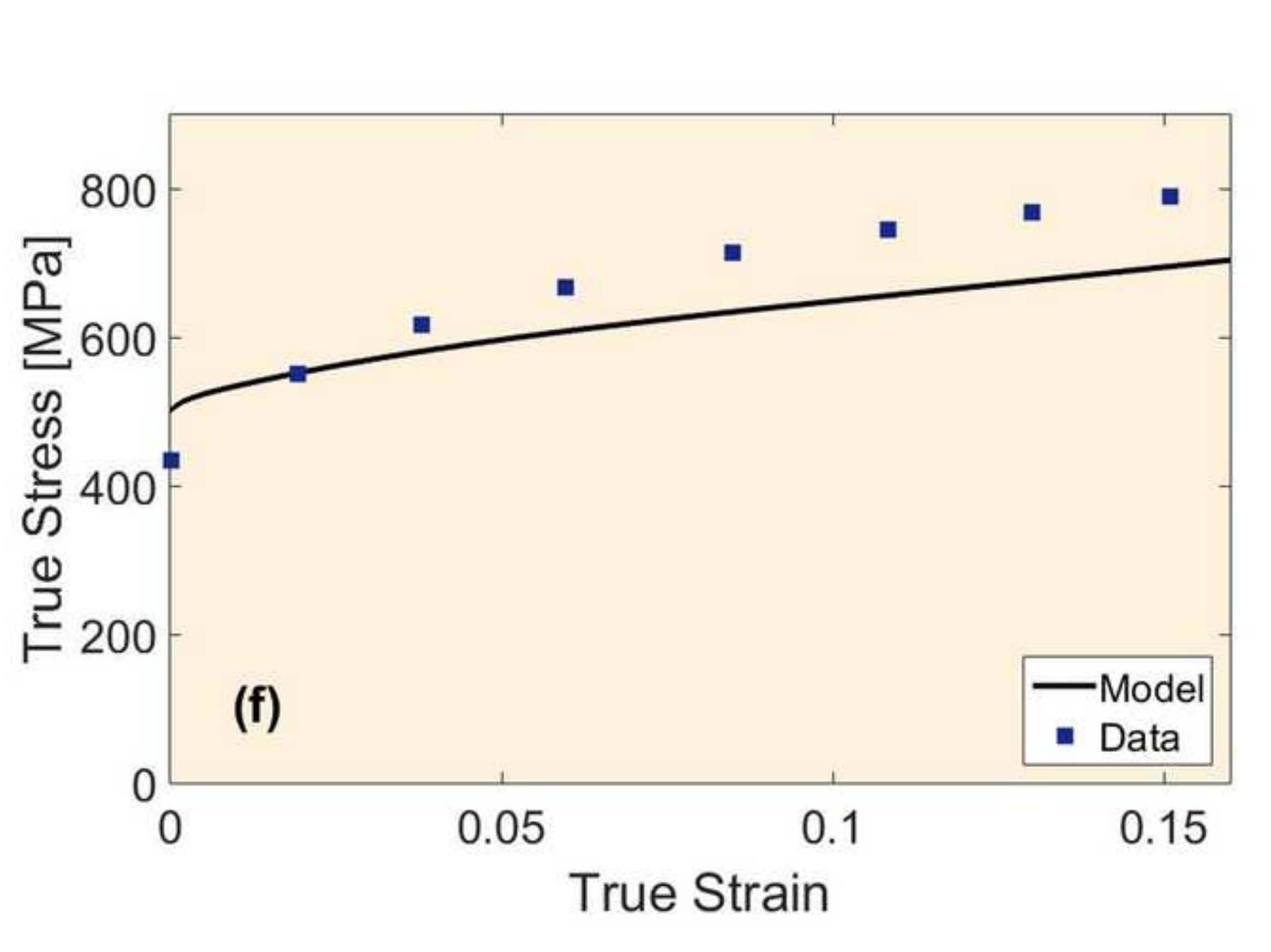

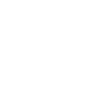

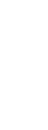
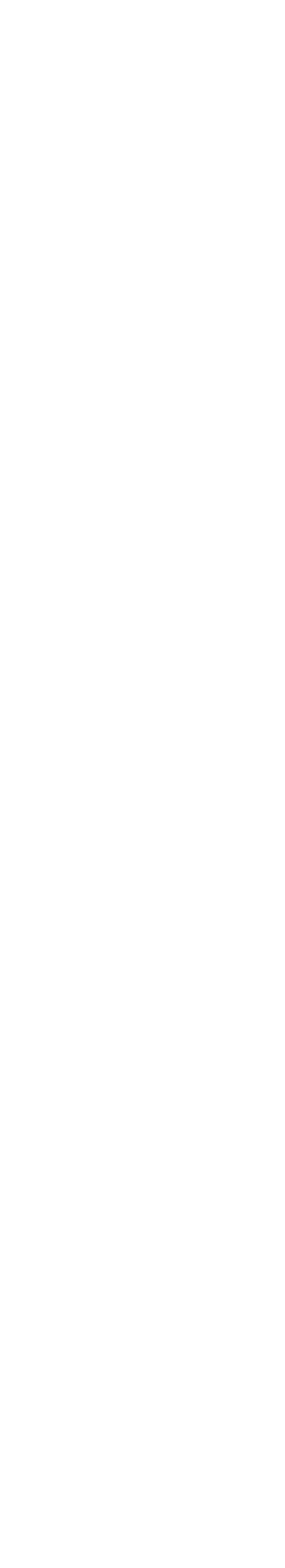


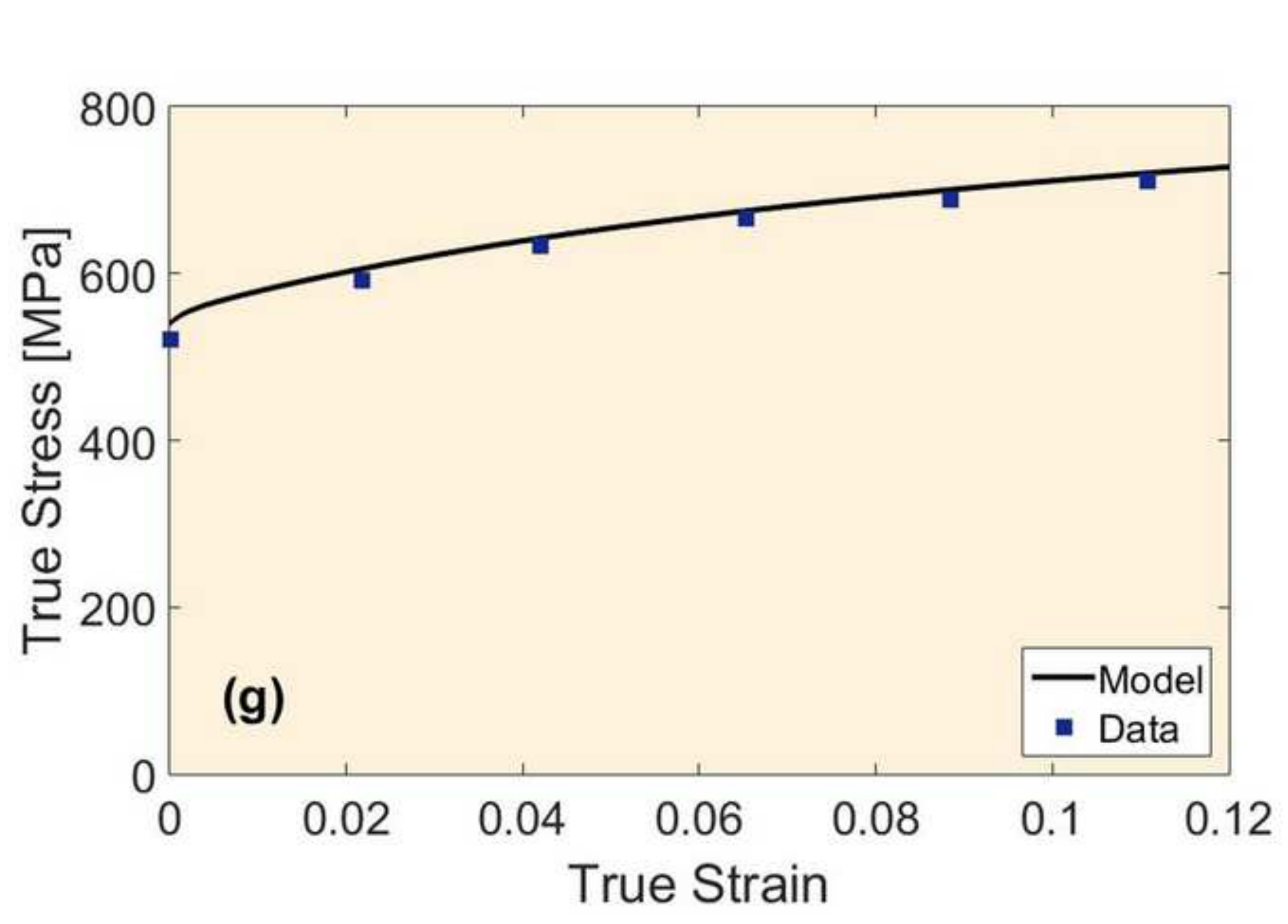

.

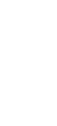




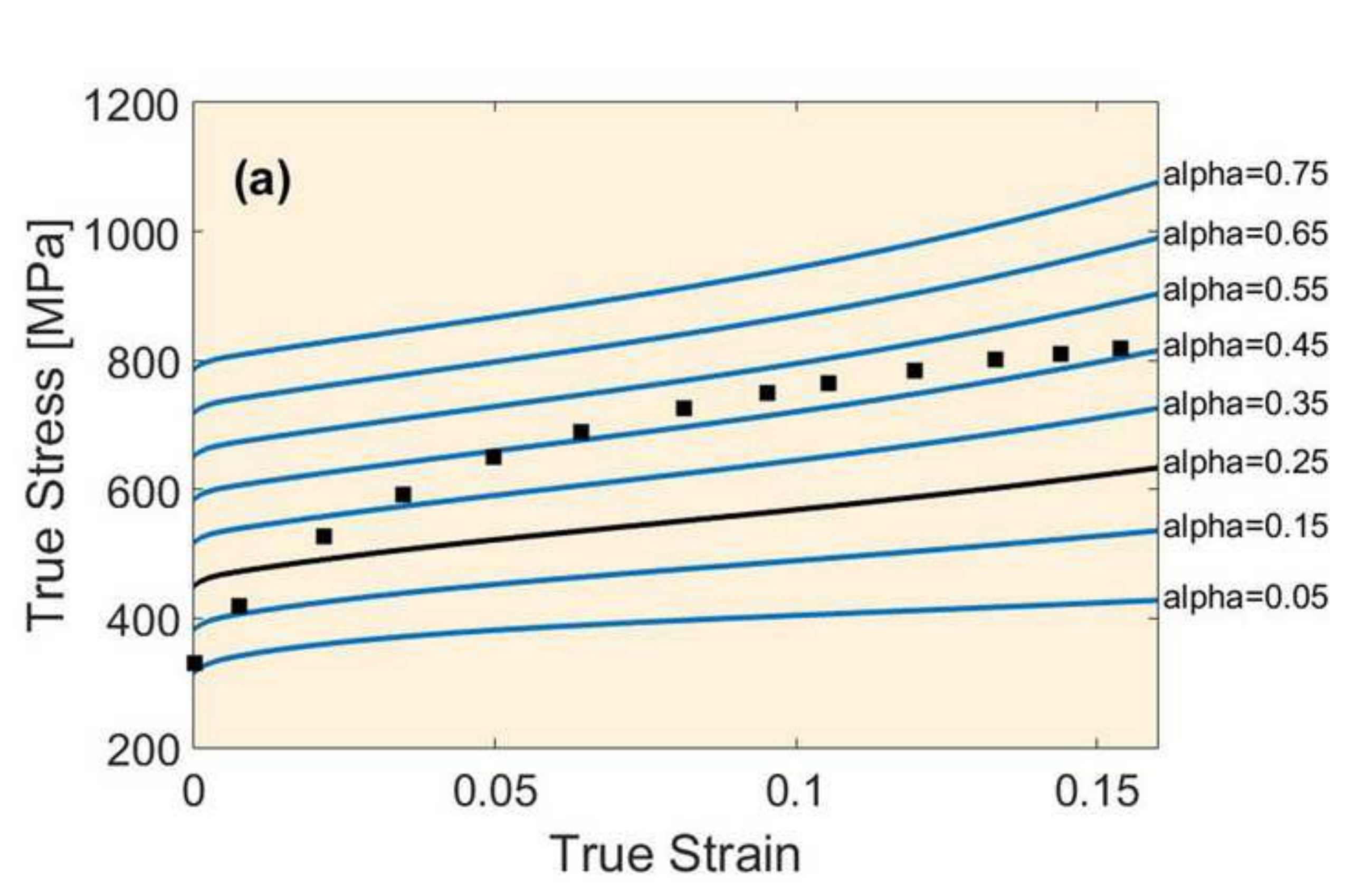




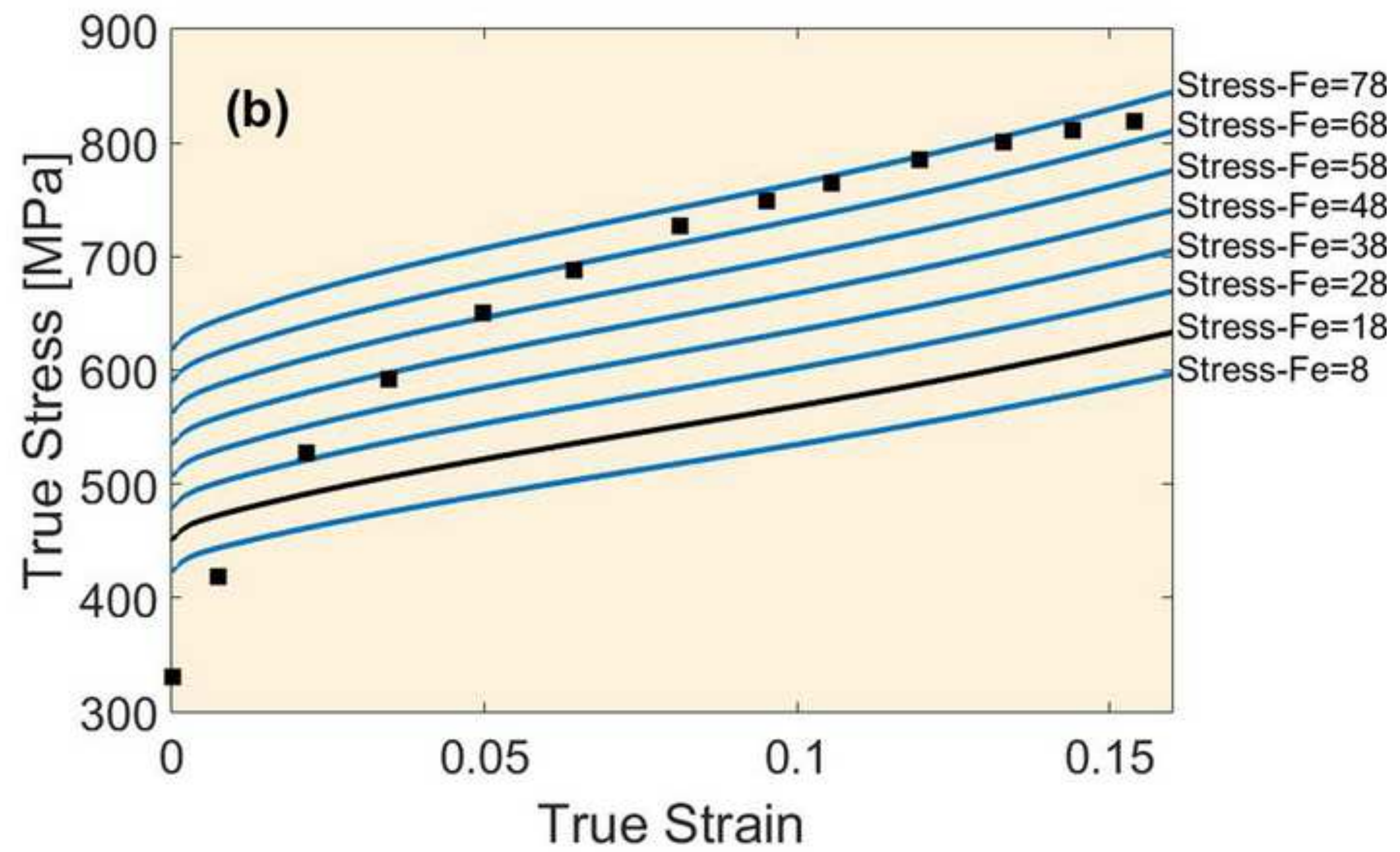




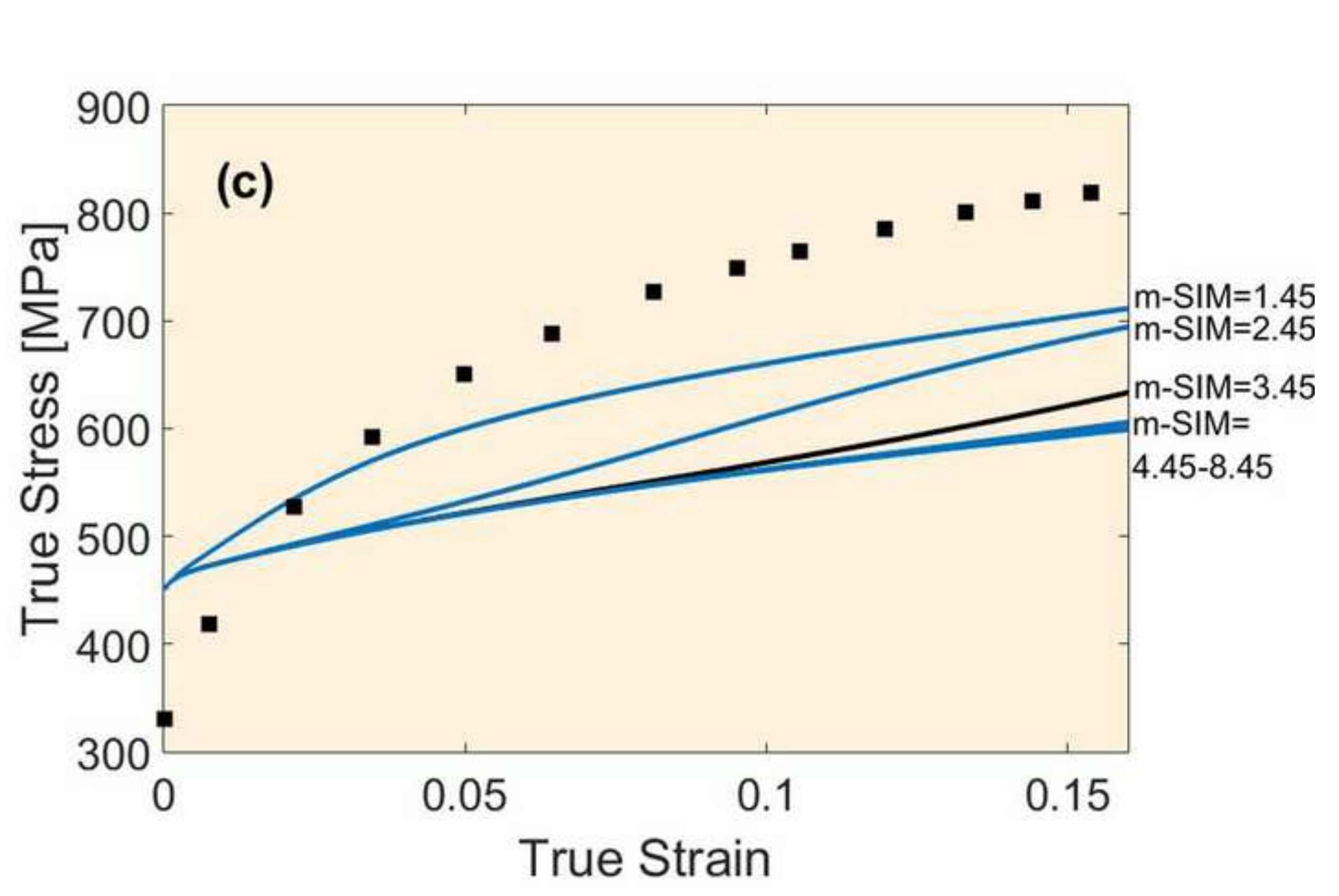




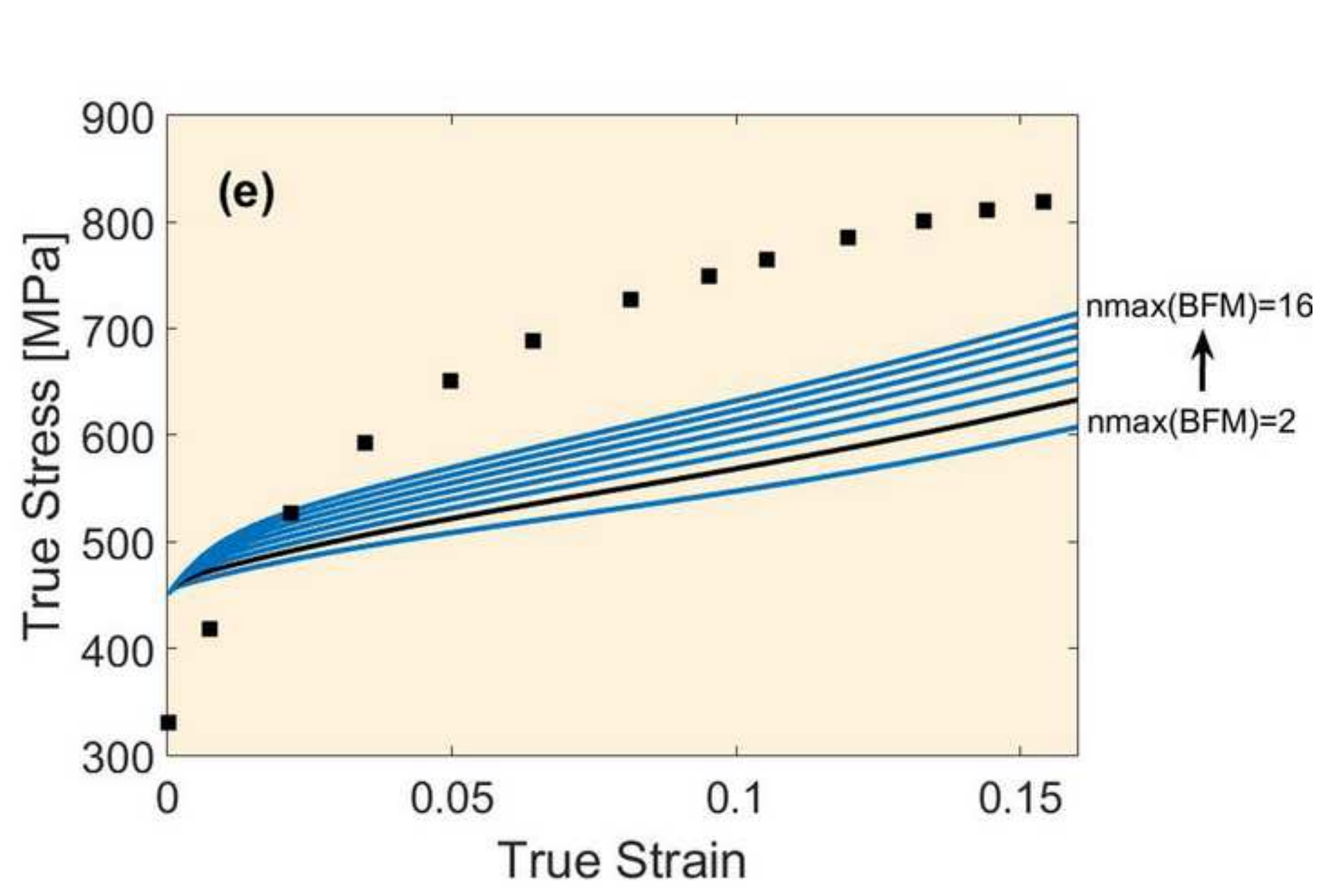

\section{True Strain}

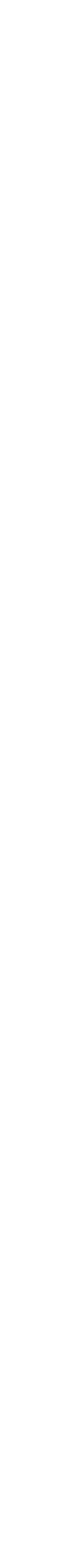



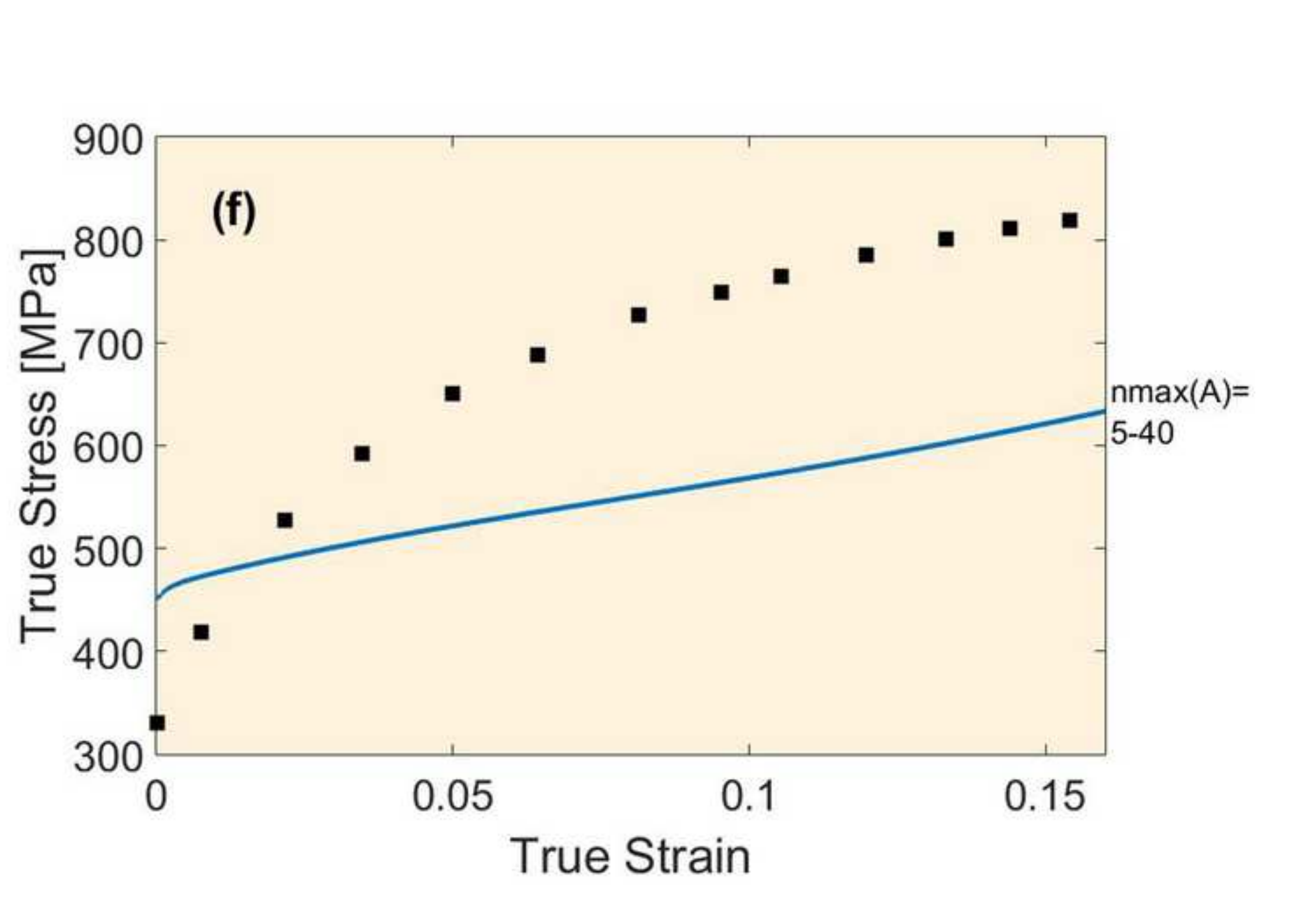

.

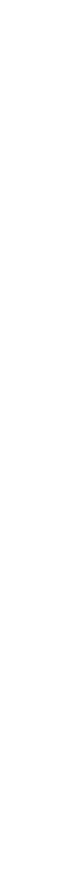




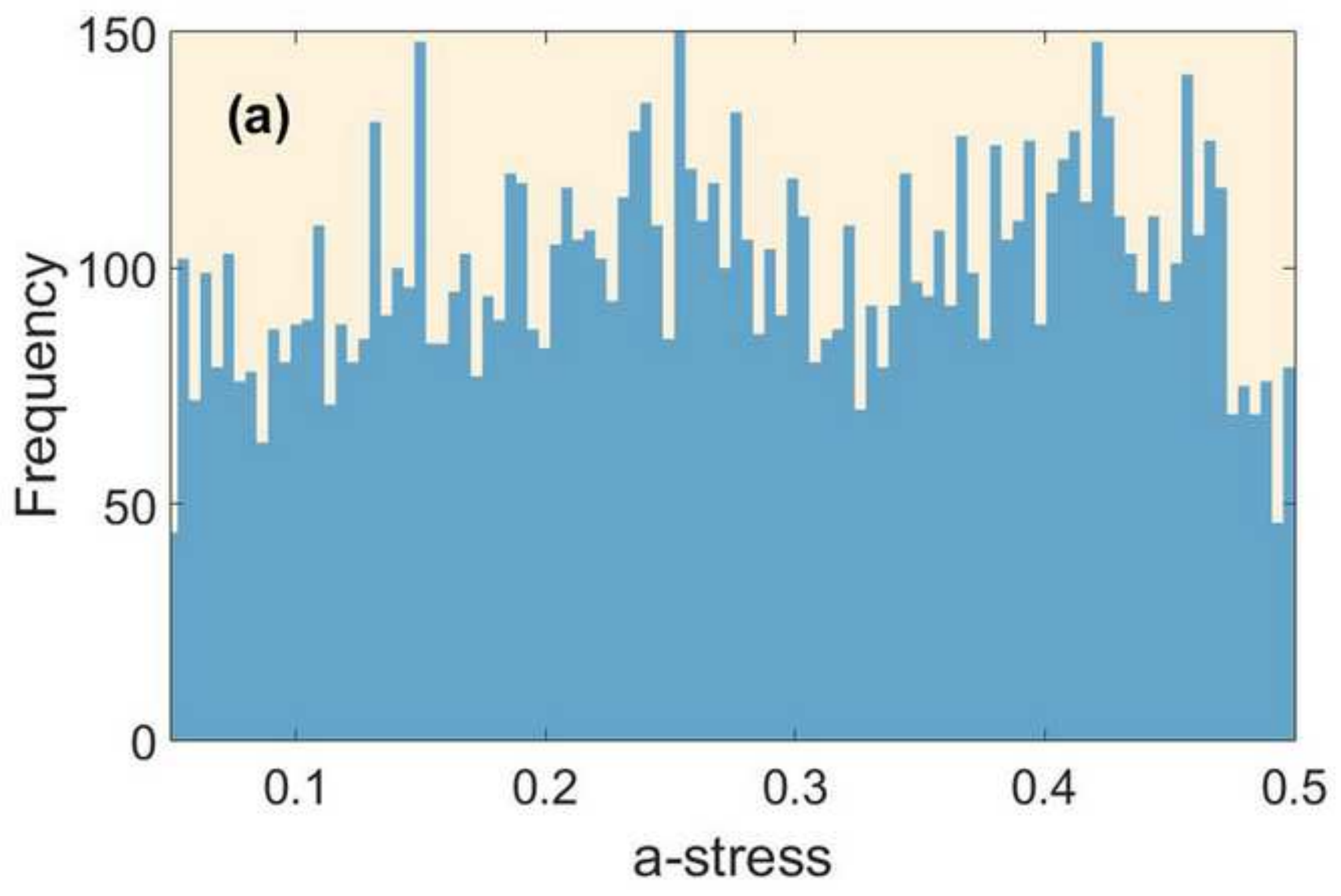




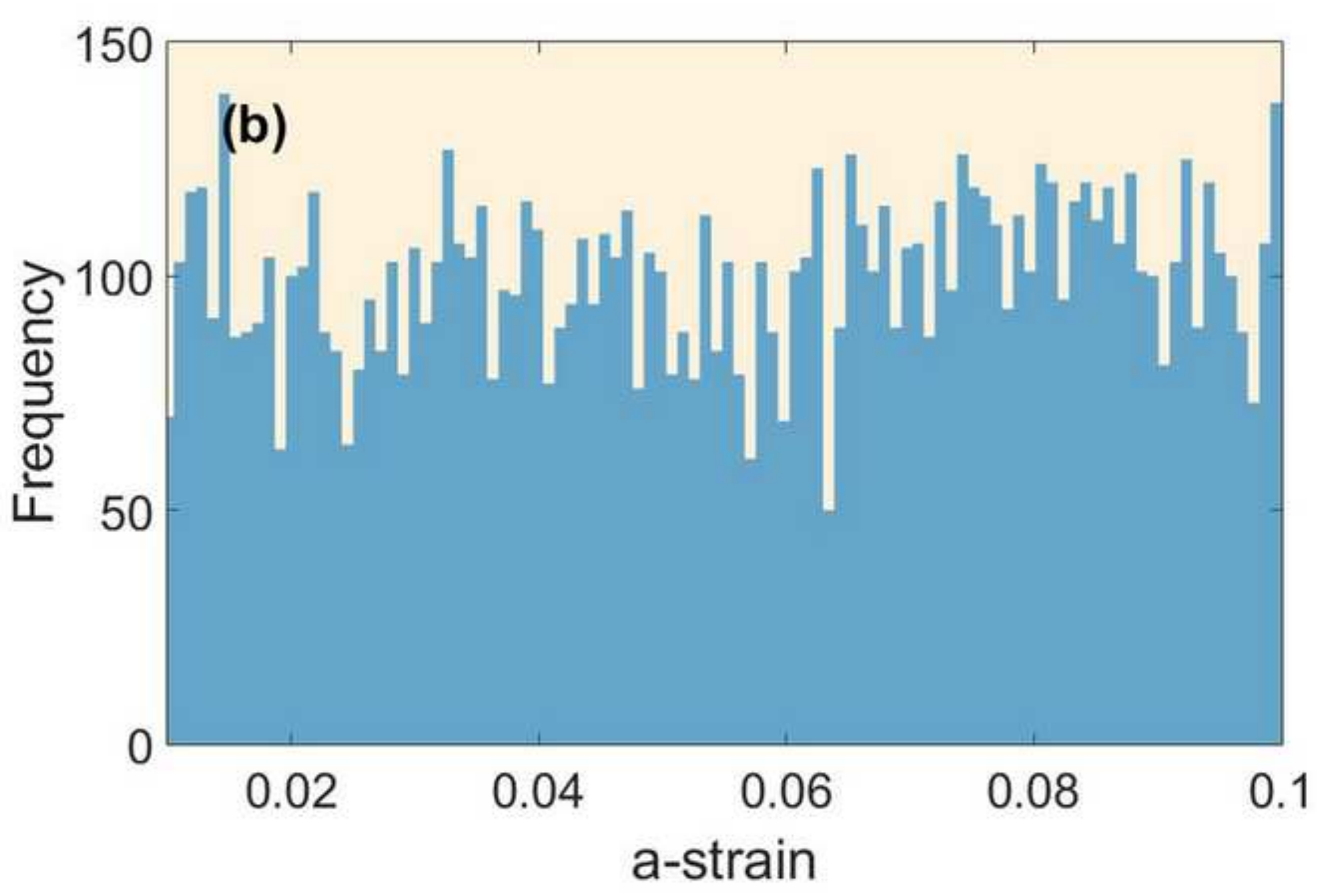




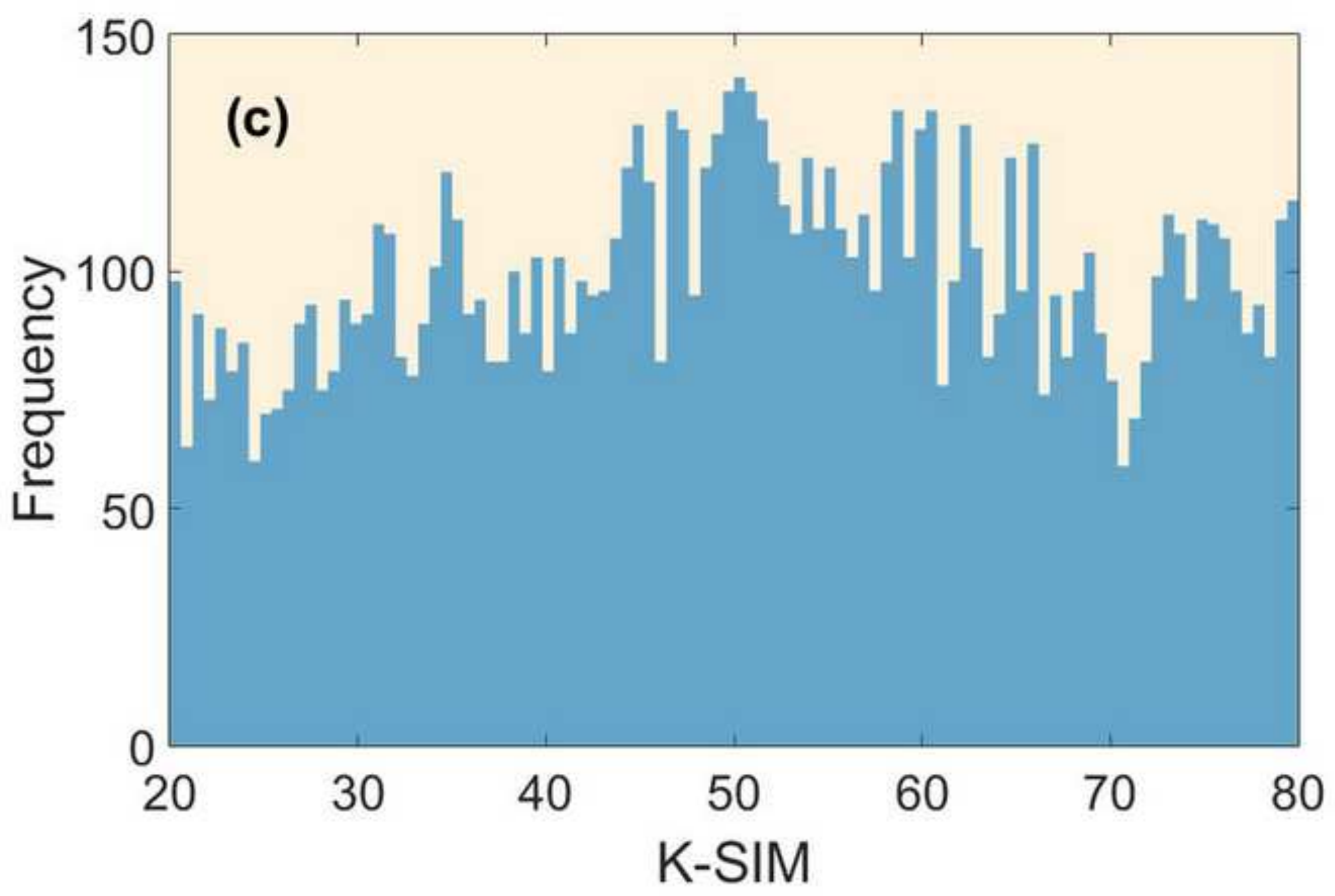




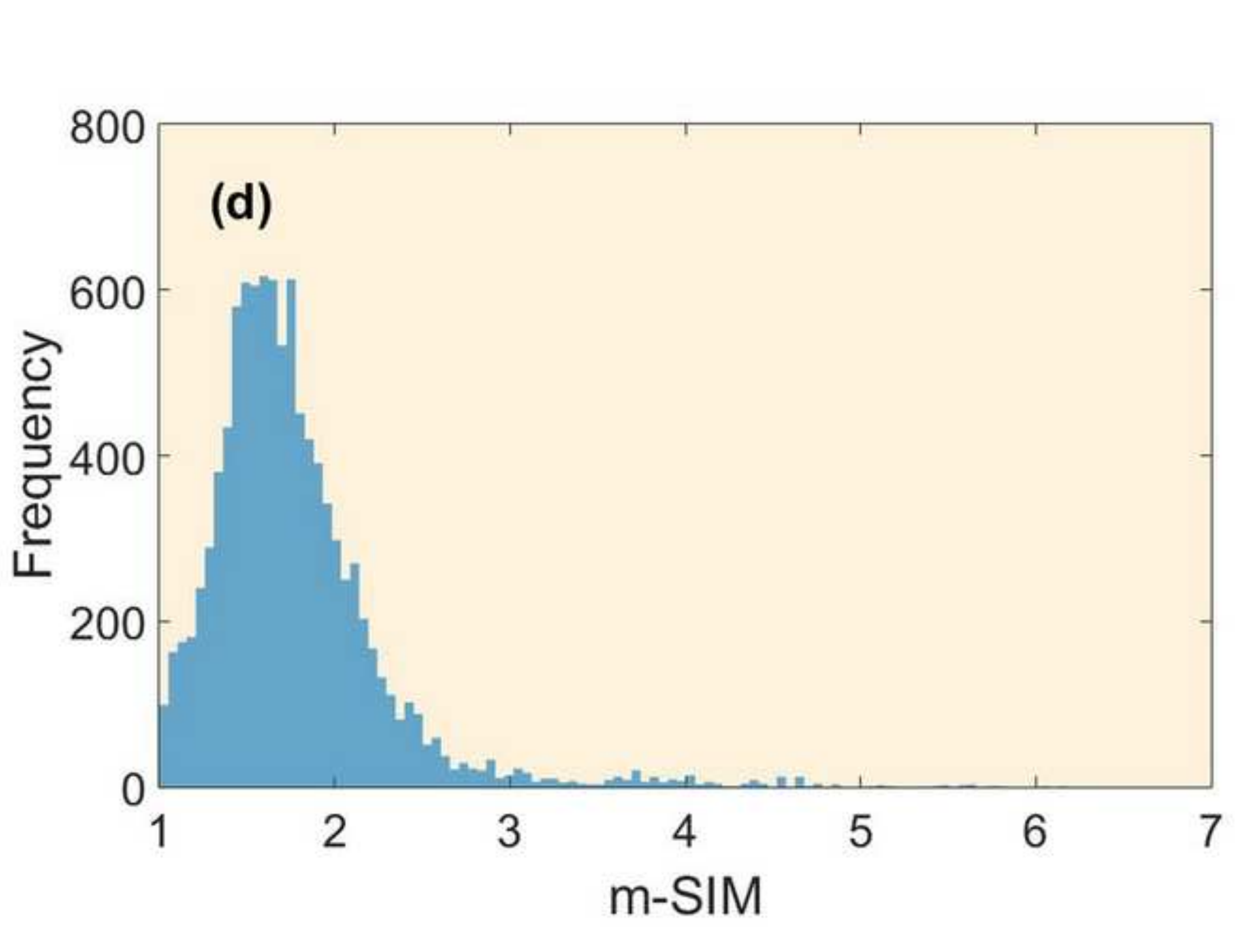

(

\author{
m-SIM
}

.

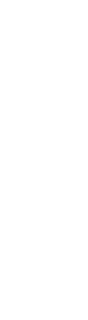

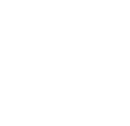

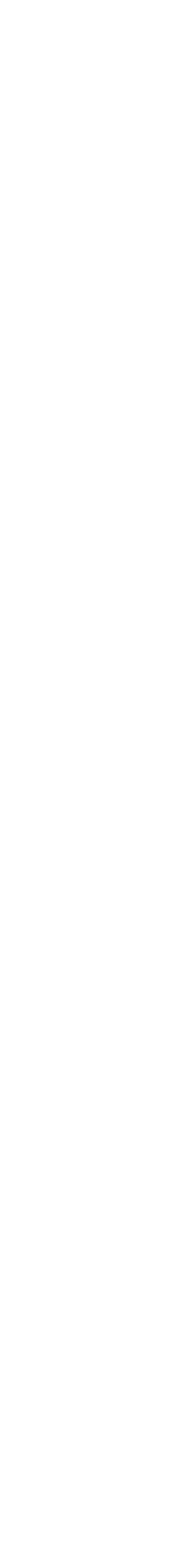

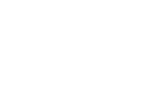




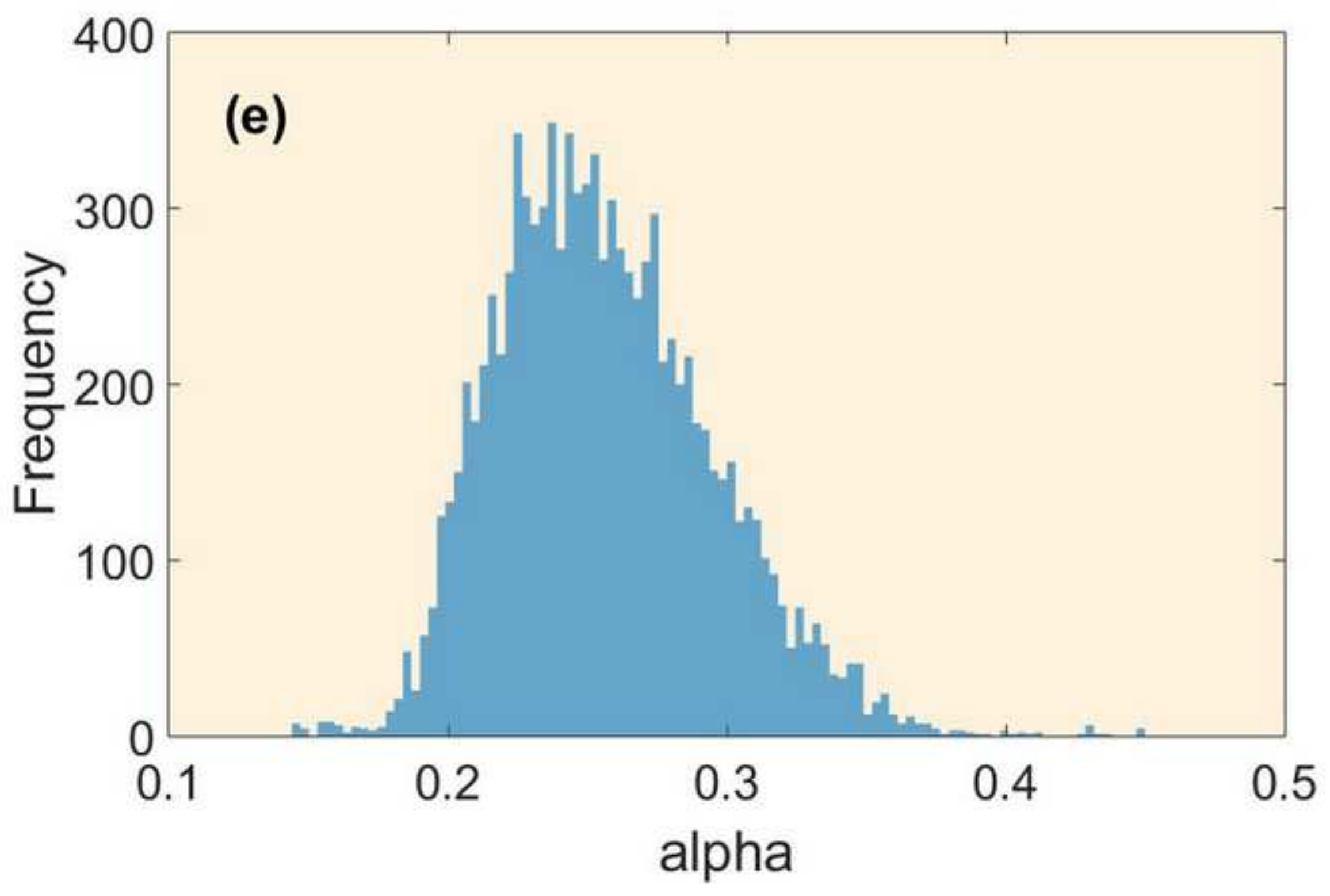




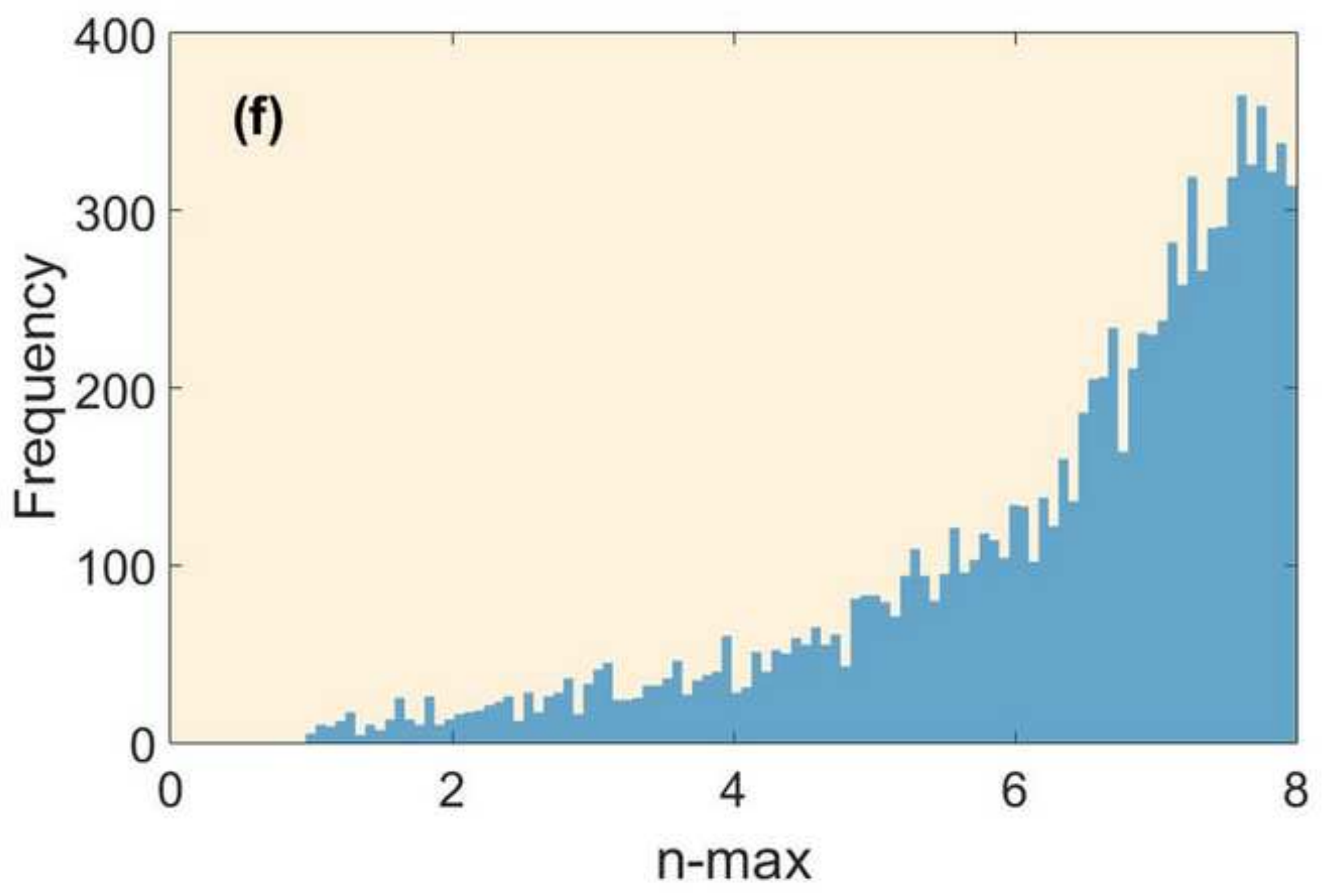




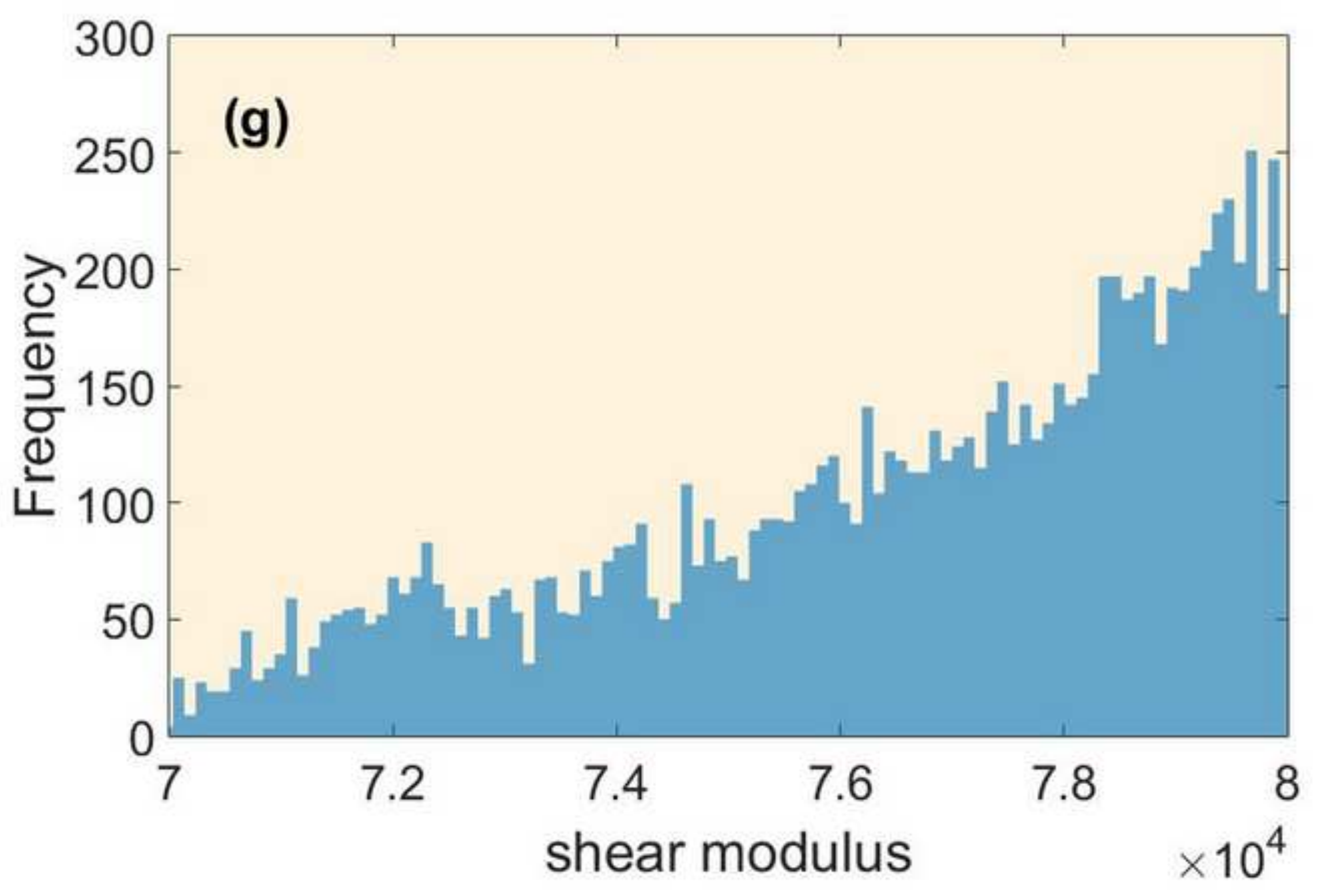




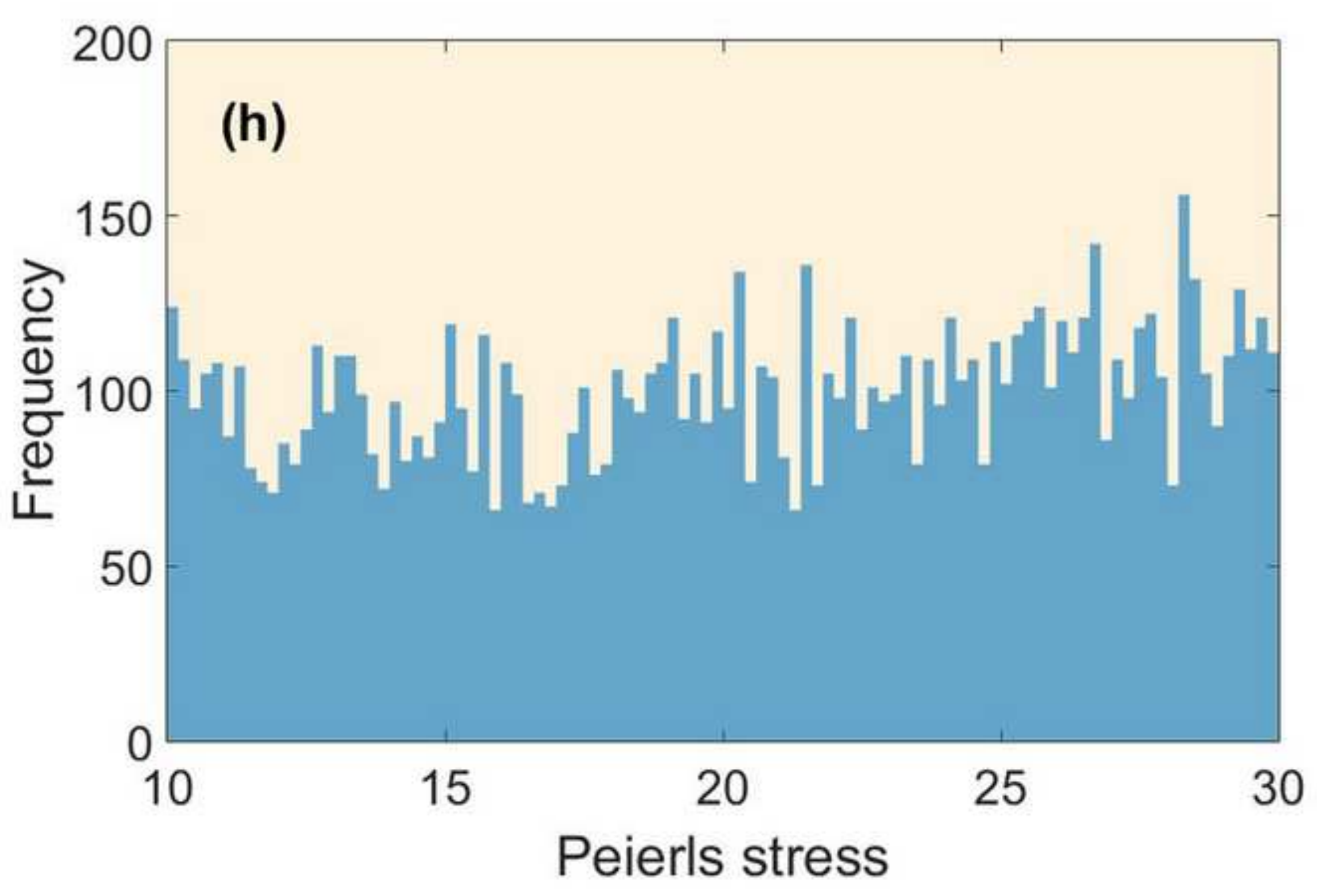




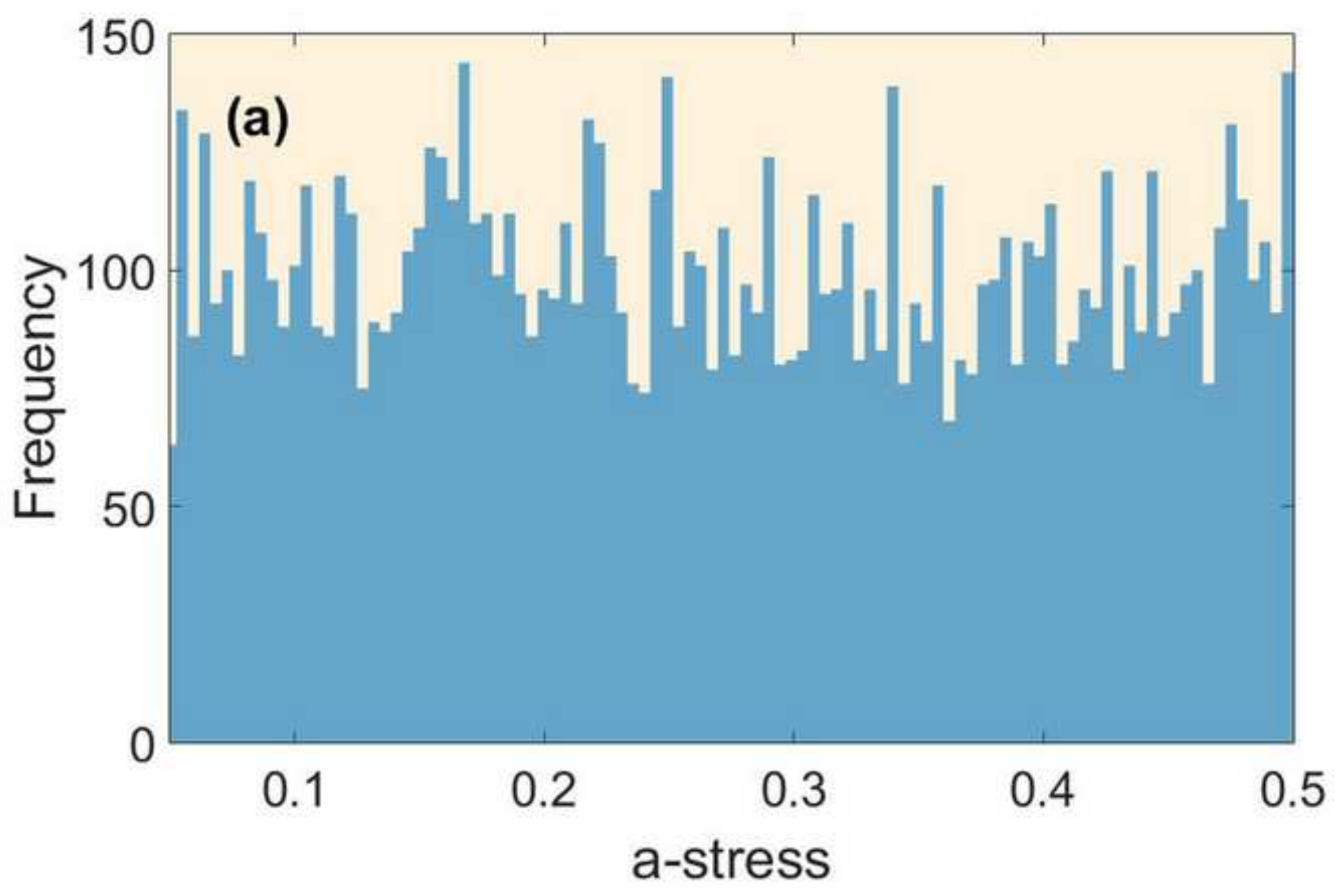




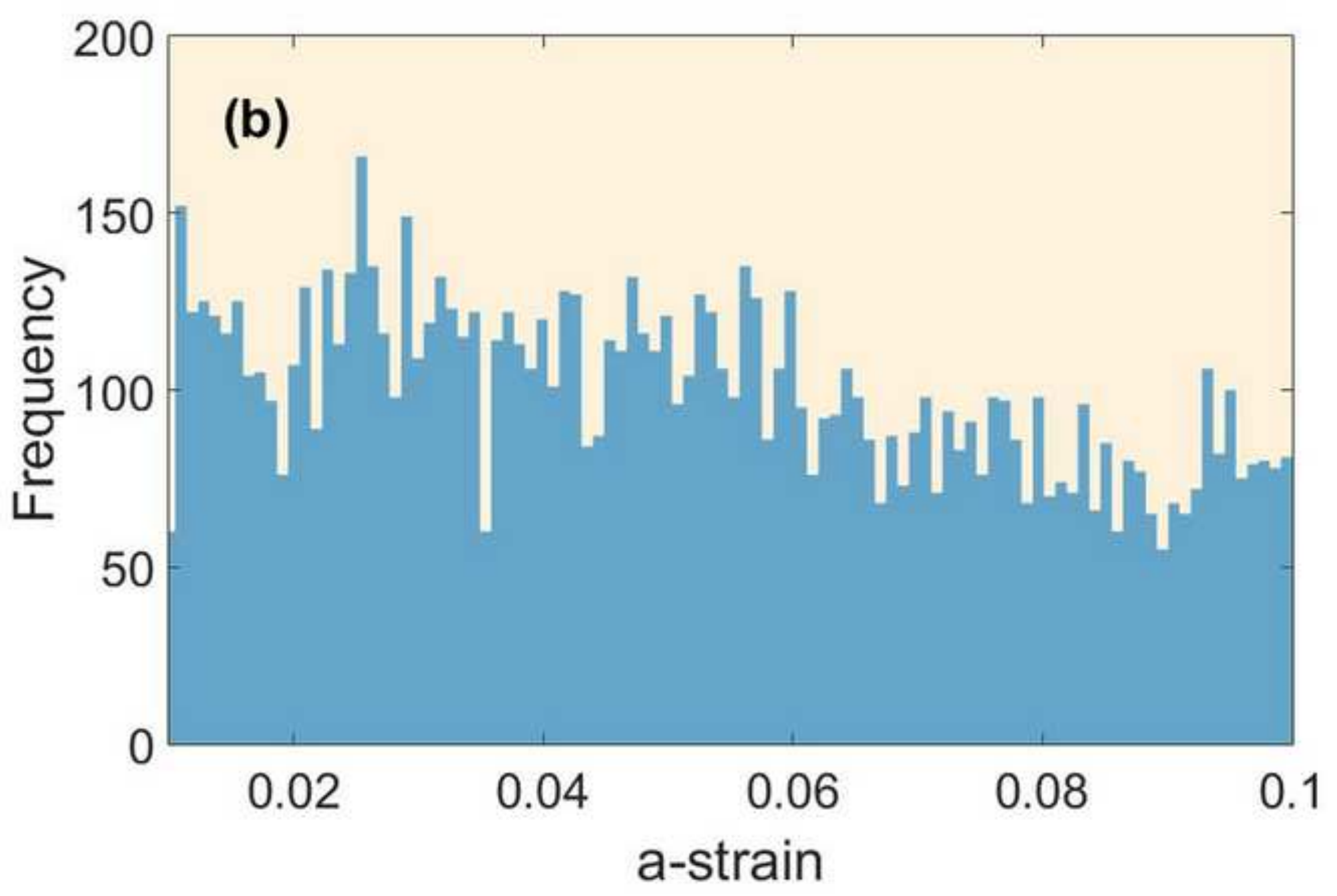




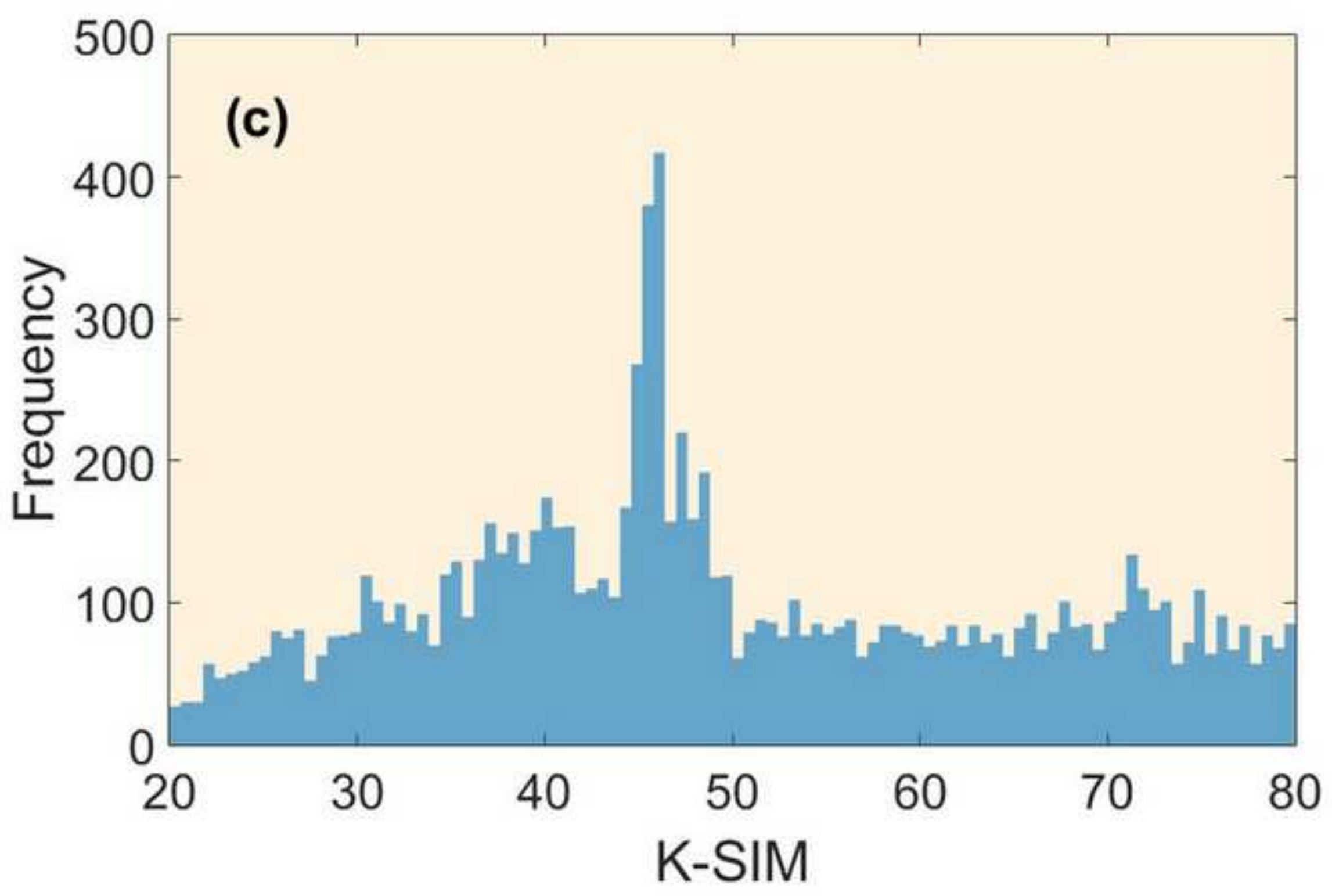




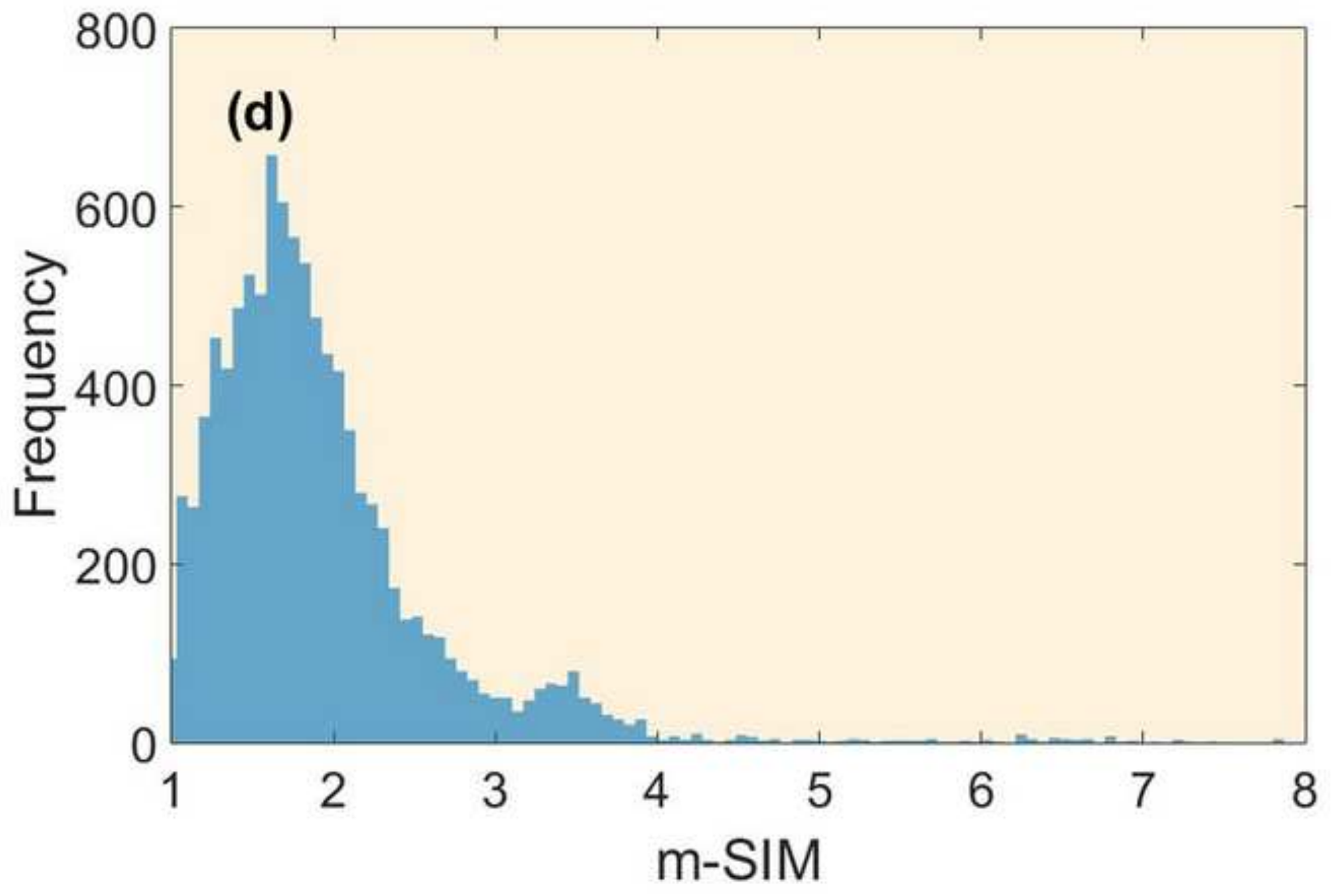




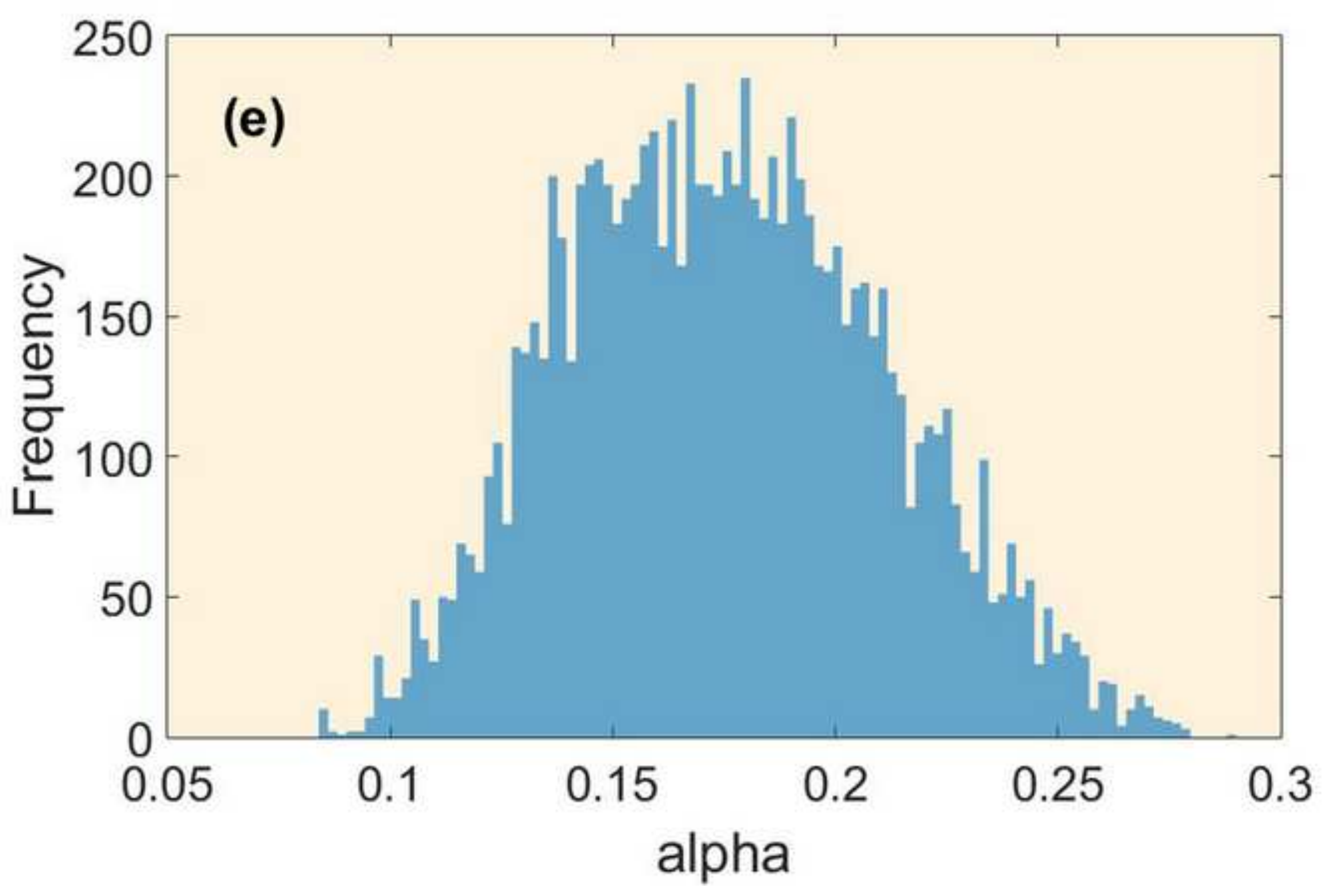




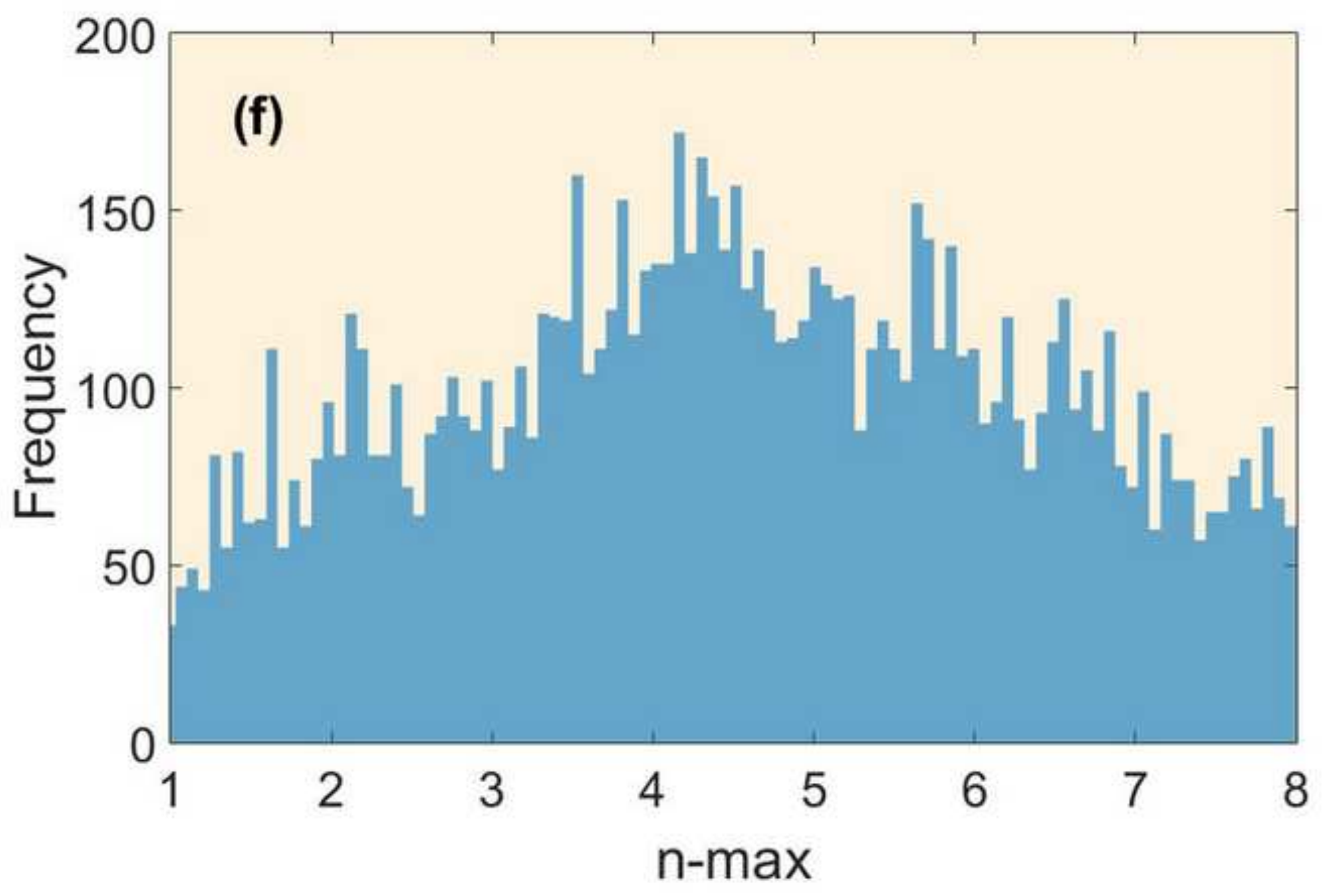




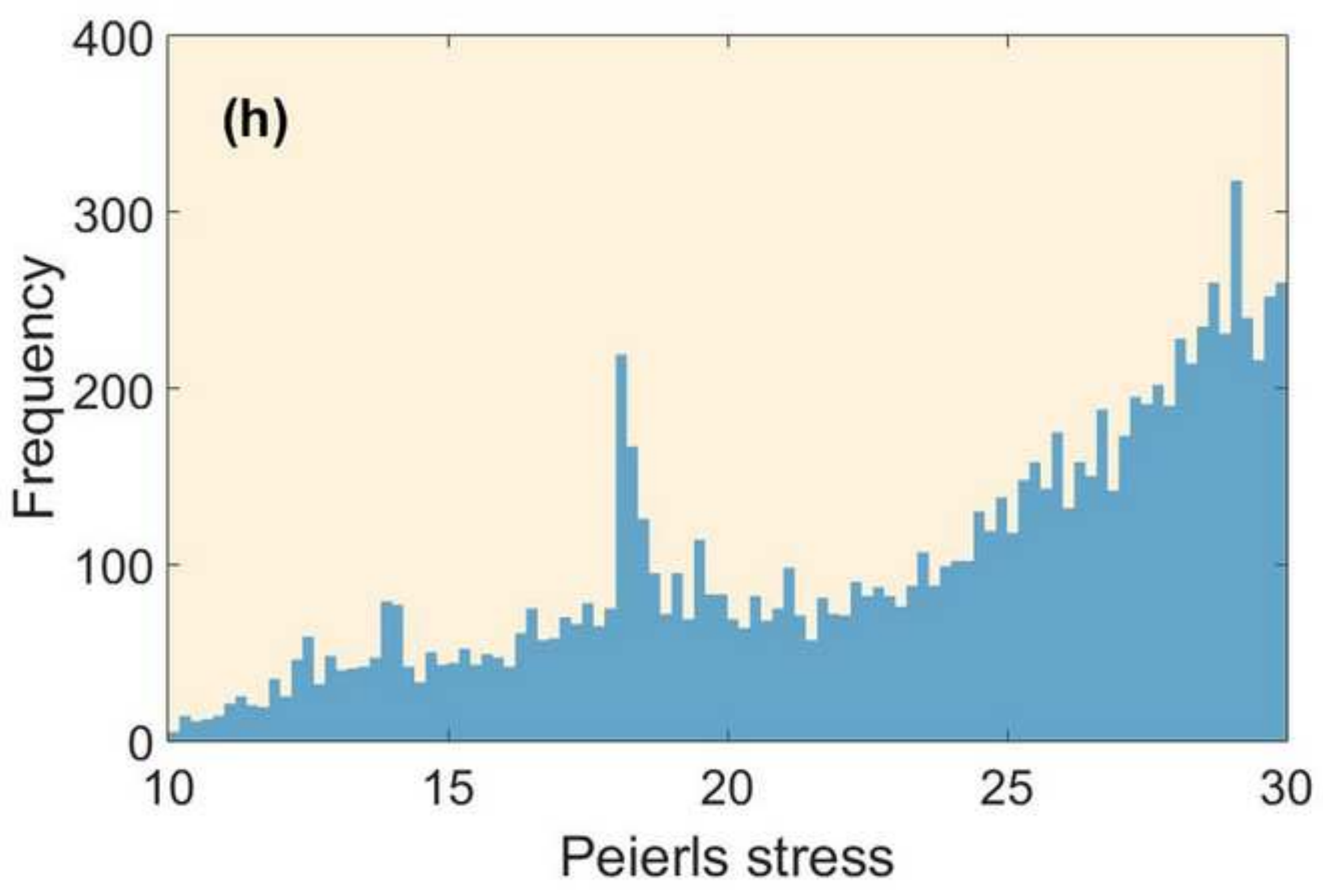




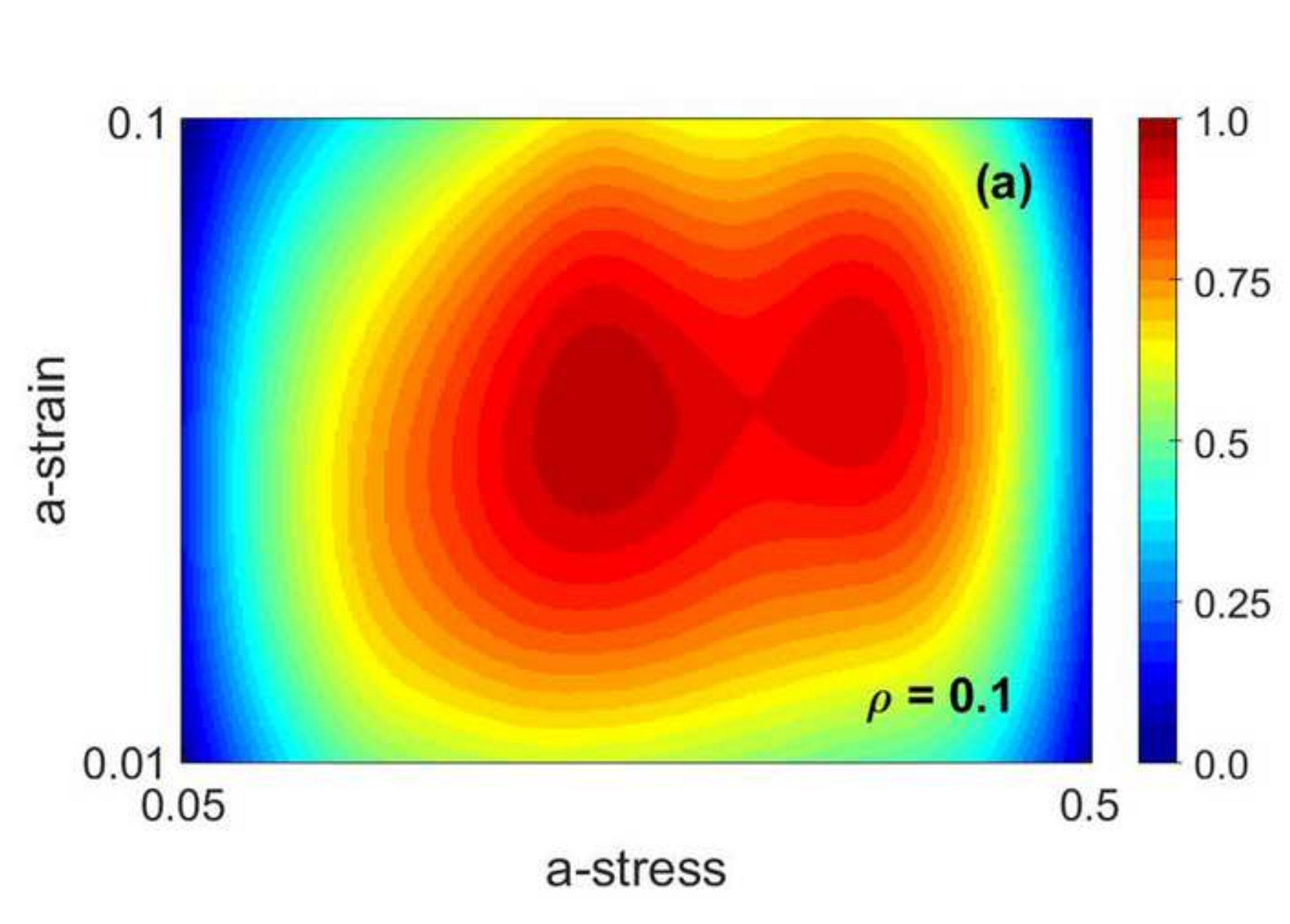

\section{a-stress}




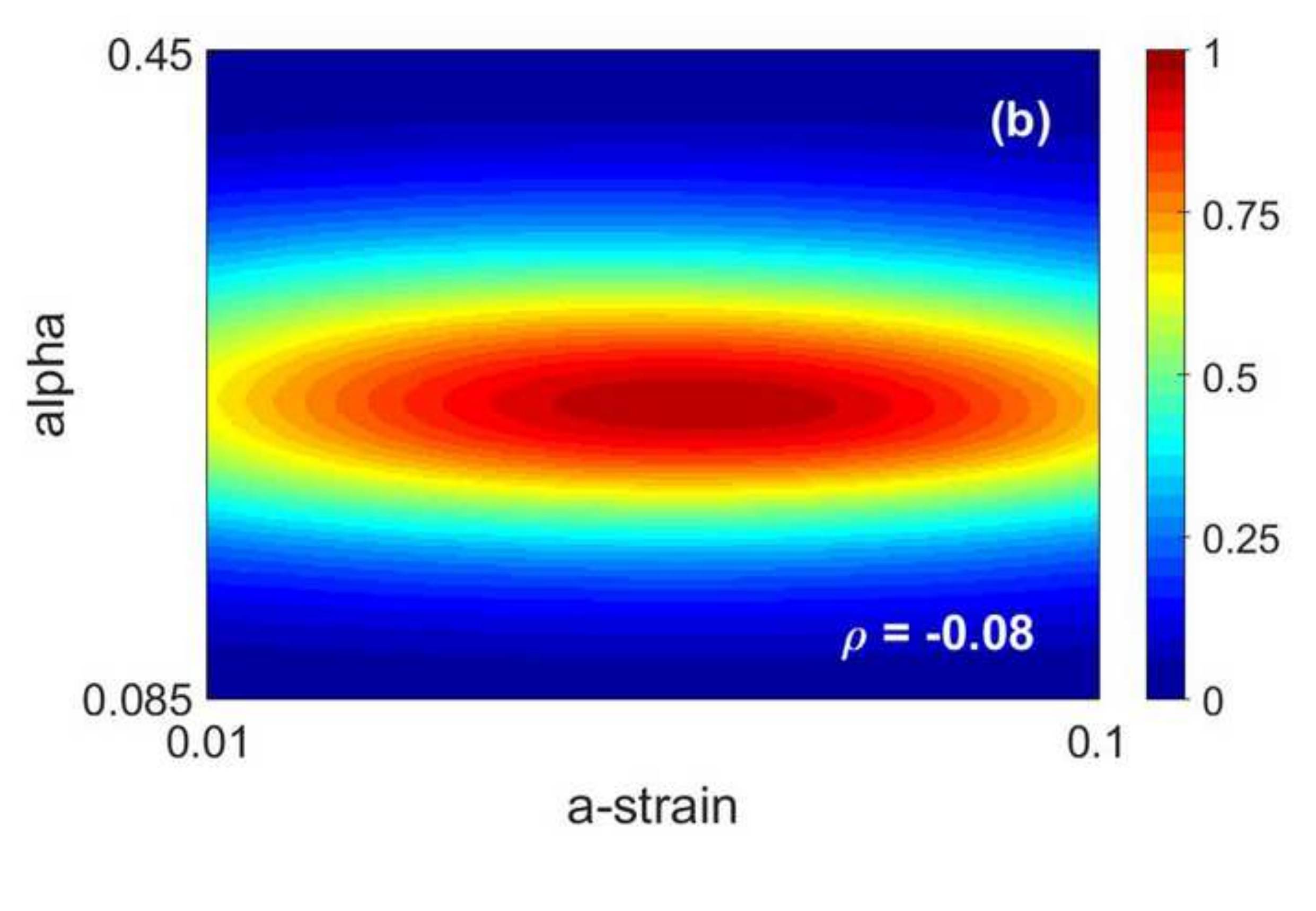

a-strain

0.085 0.01

$$
\rho=-0.08
$$

0.1

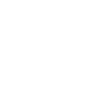

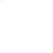

$\frac{\frac{\sigma}{\sigma}}{\frac{0}{\sigma}}$

\subsection{5}

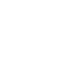

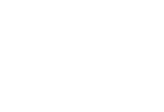




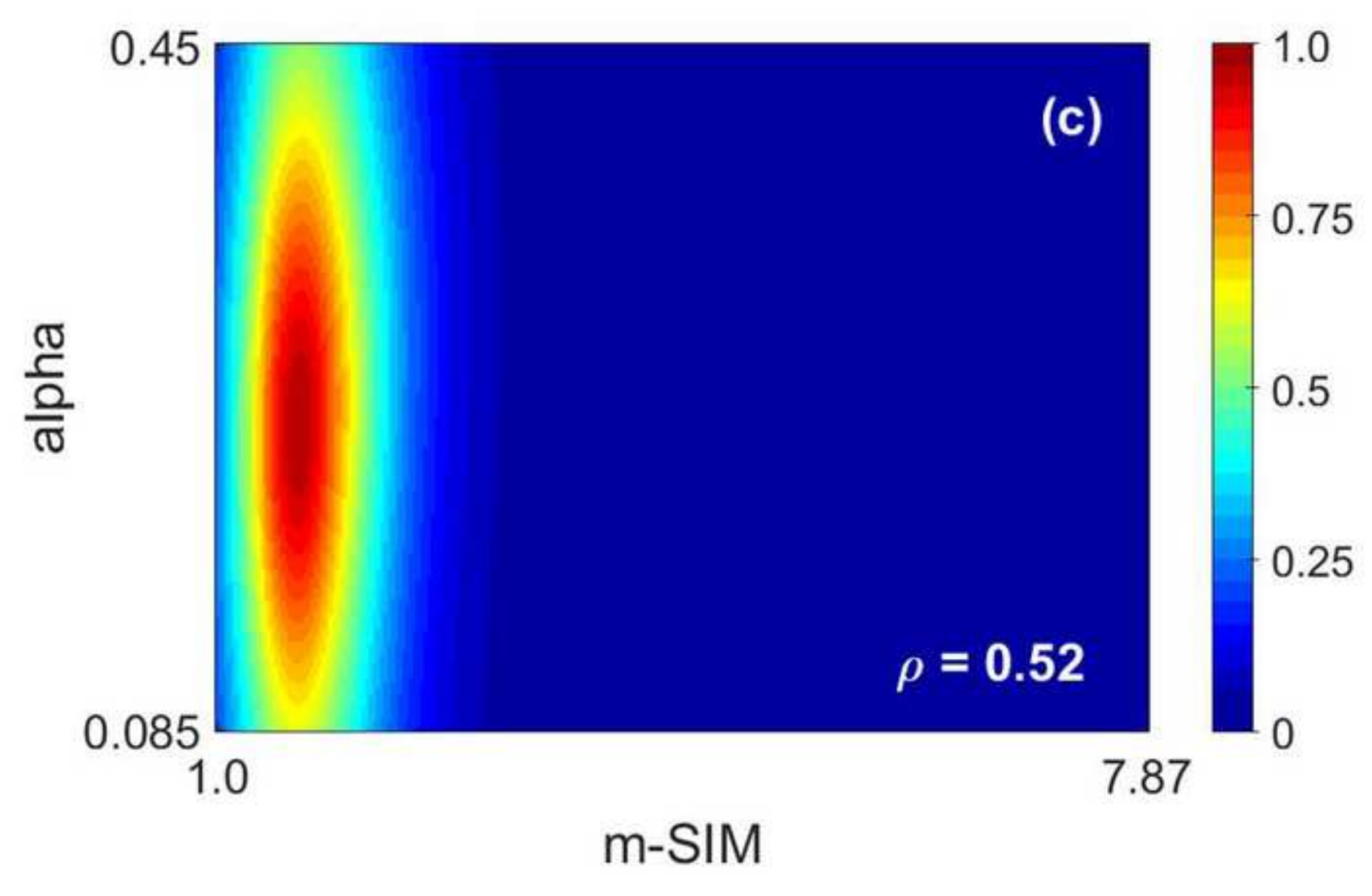

$$
\rho=0.52
$$

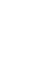

\subsection{5}

0.085

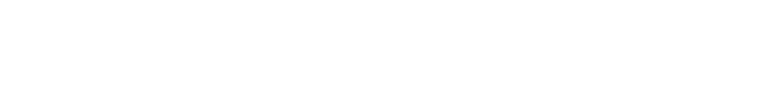




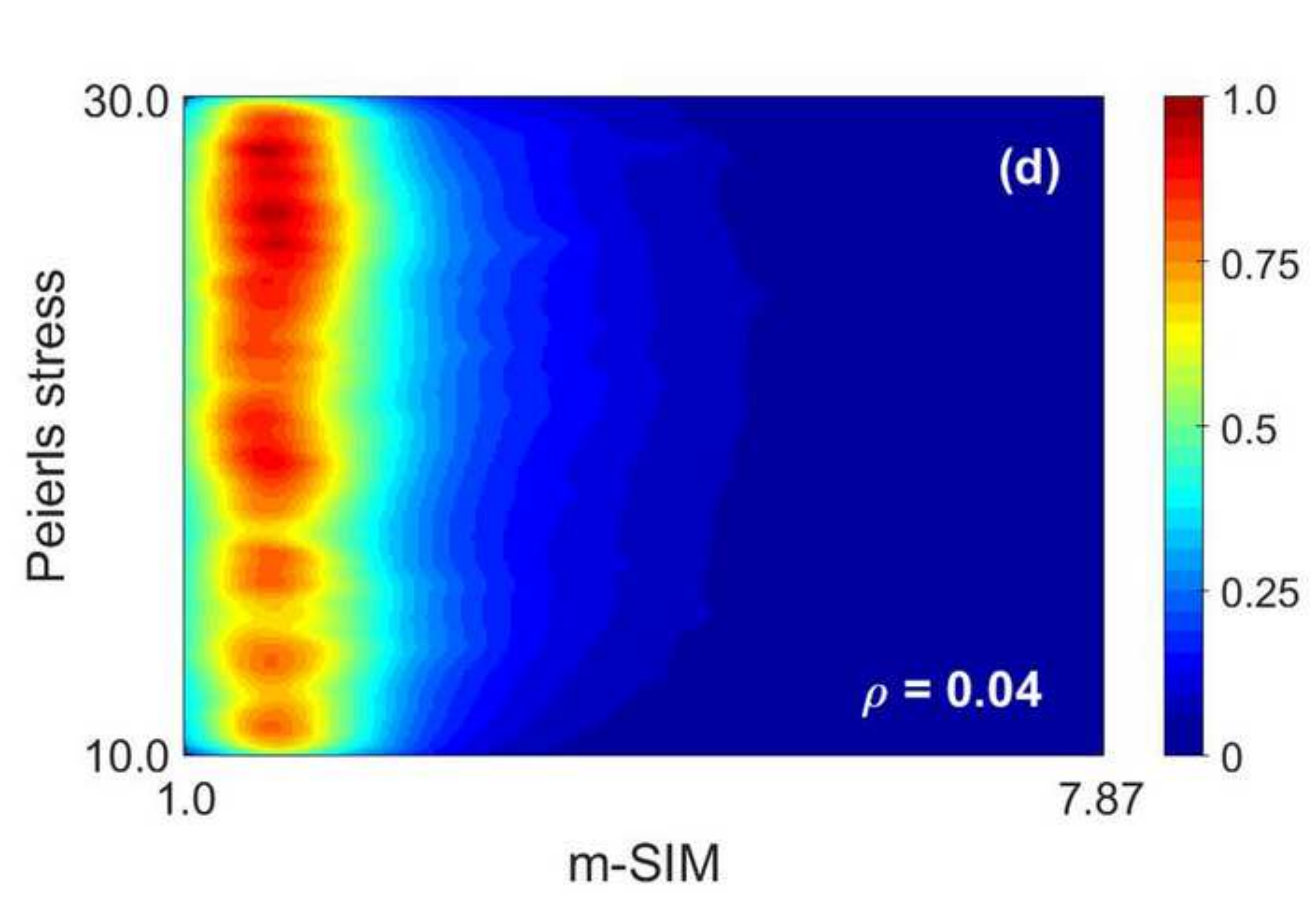

m-SIM 


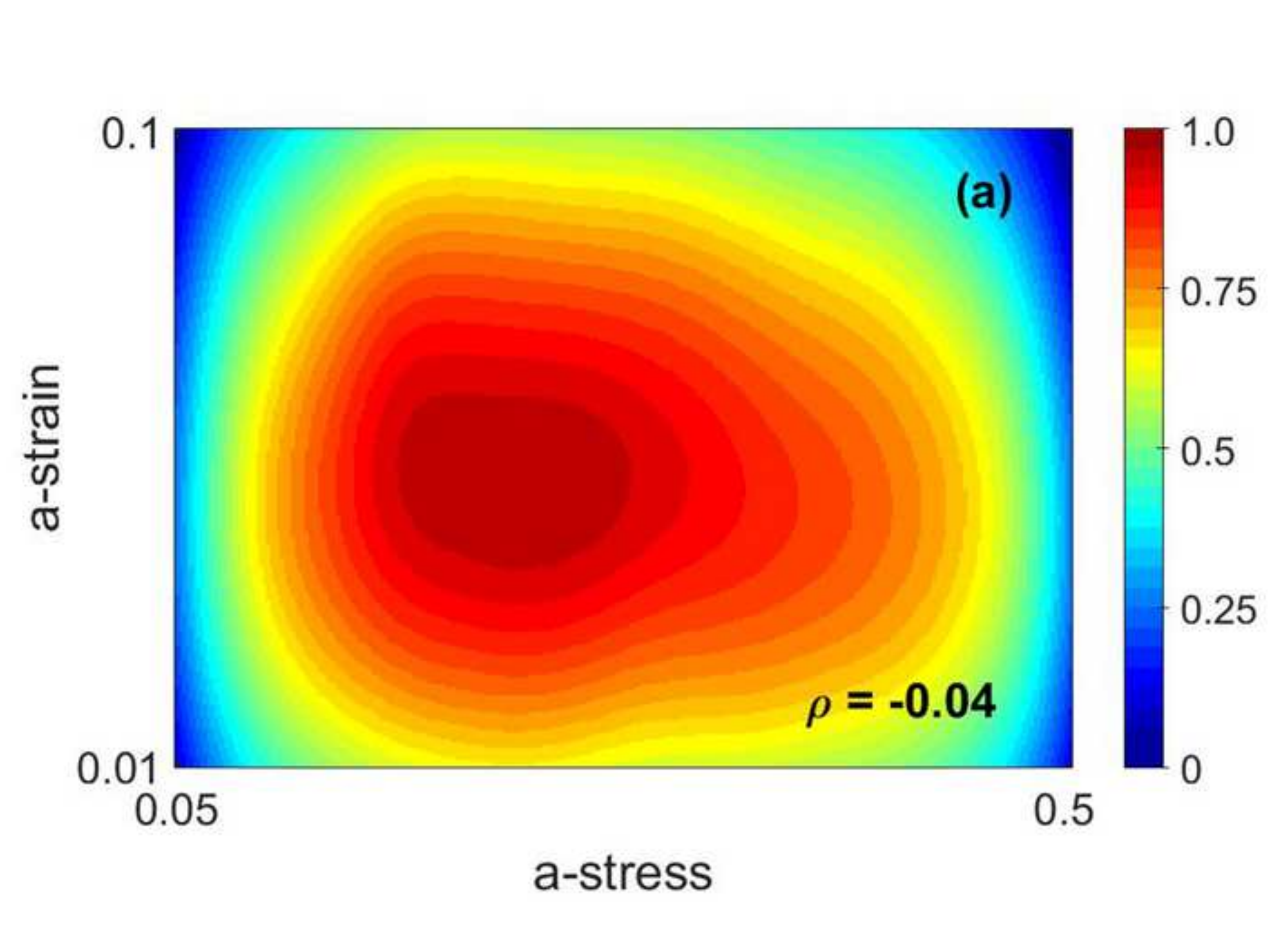

a-stress

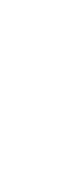




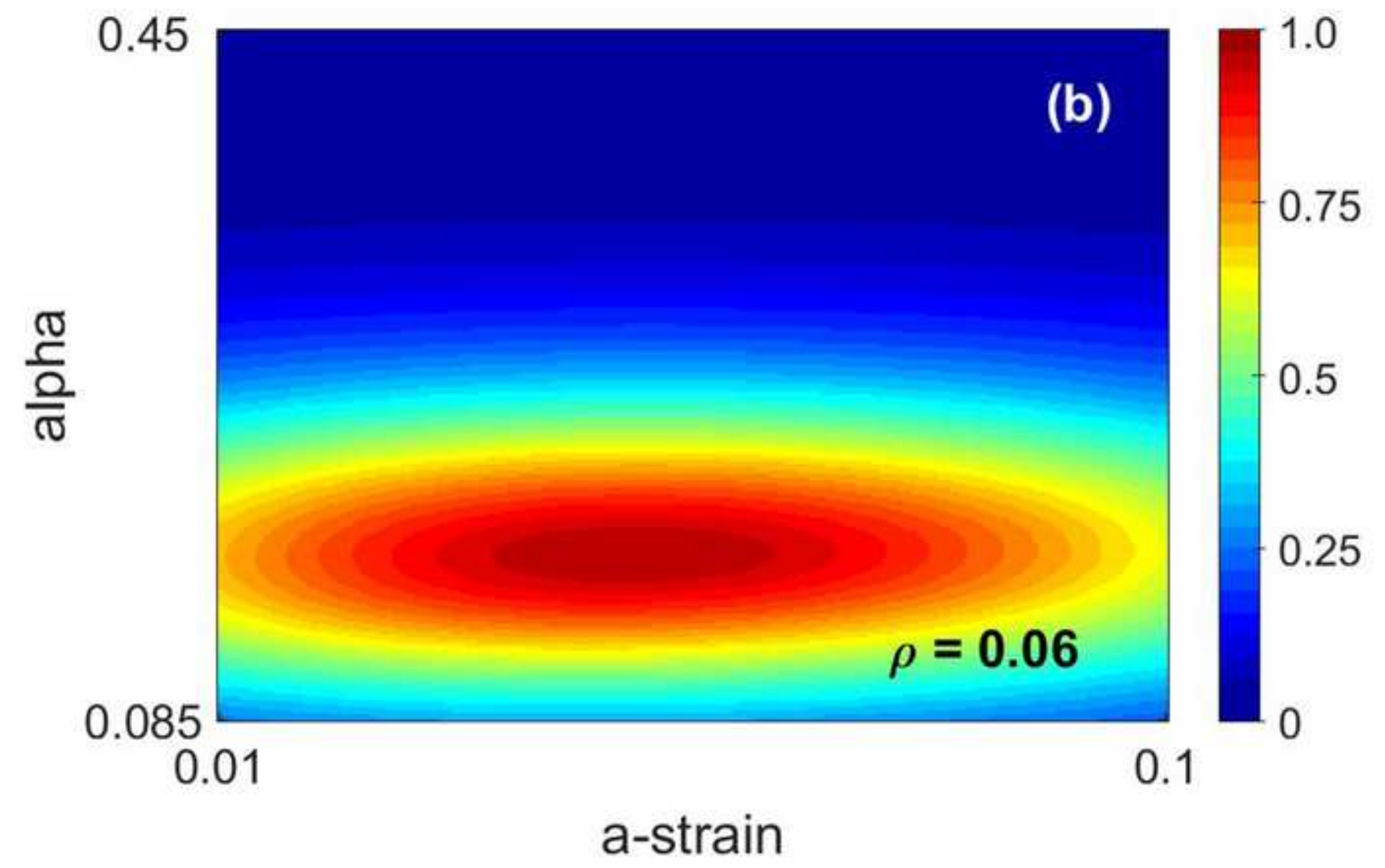

(b)

\section{$\rho=0.06$}

0.1

.

0.45

$\frac{\text { 뭉 }}{\frac{2}{\sigma}}$

\section{a-strain}

0.085
0.01

a-strain

.




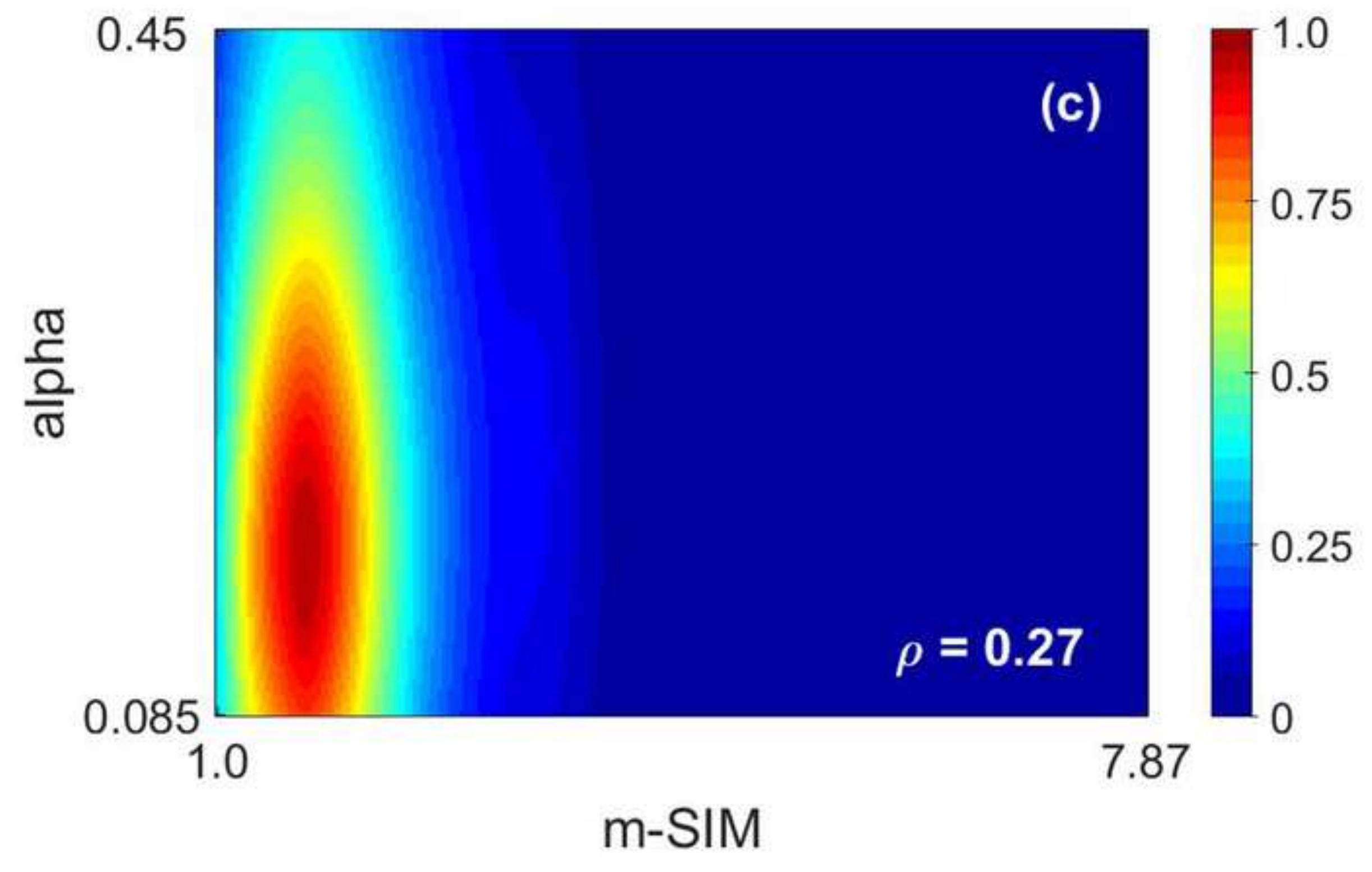

m-SIM

$$
\rho=0.27
$$

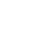

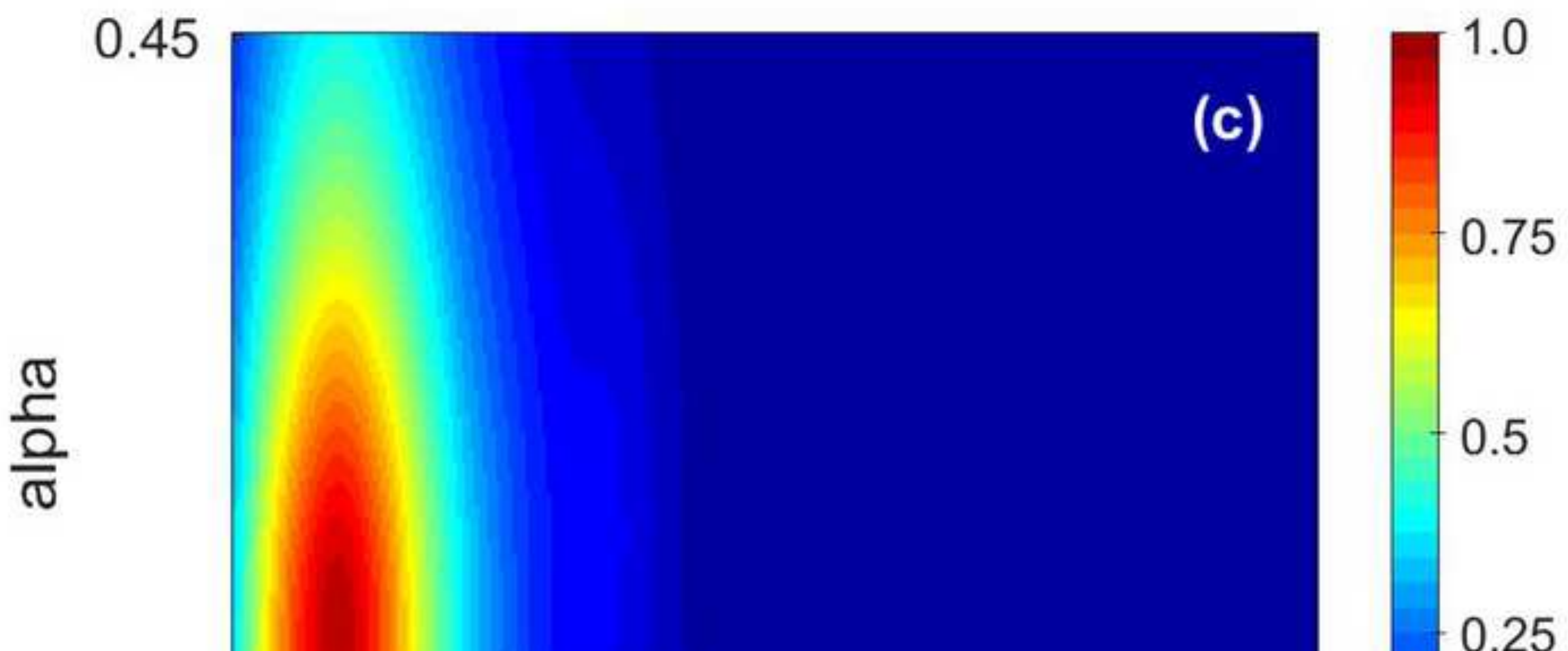




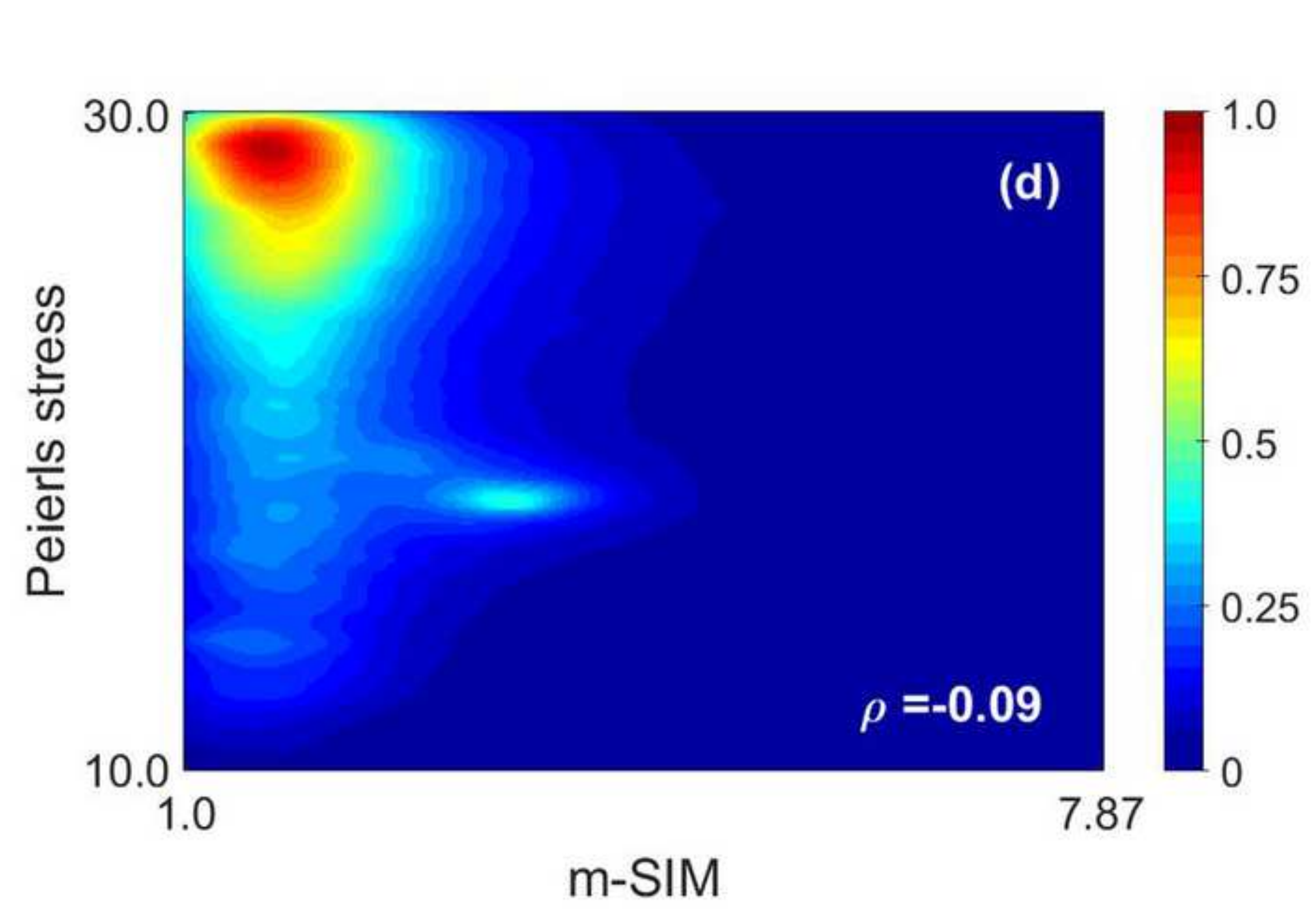

m-SIM 


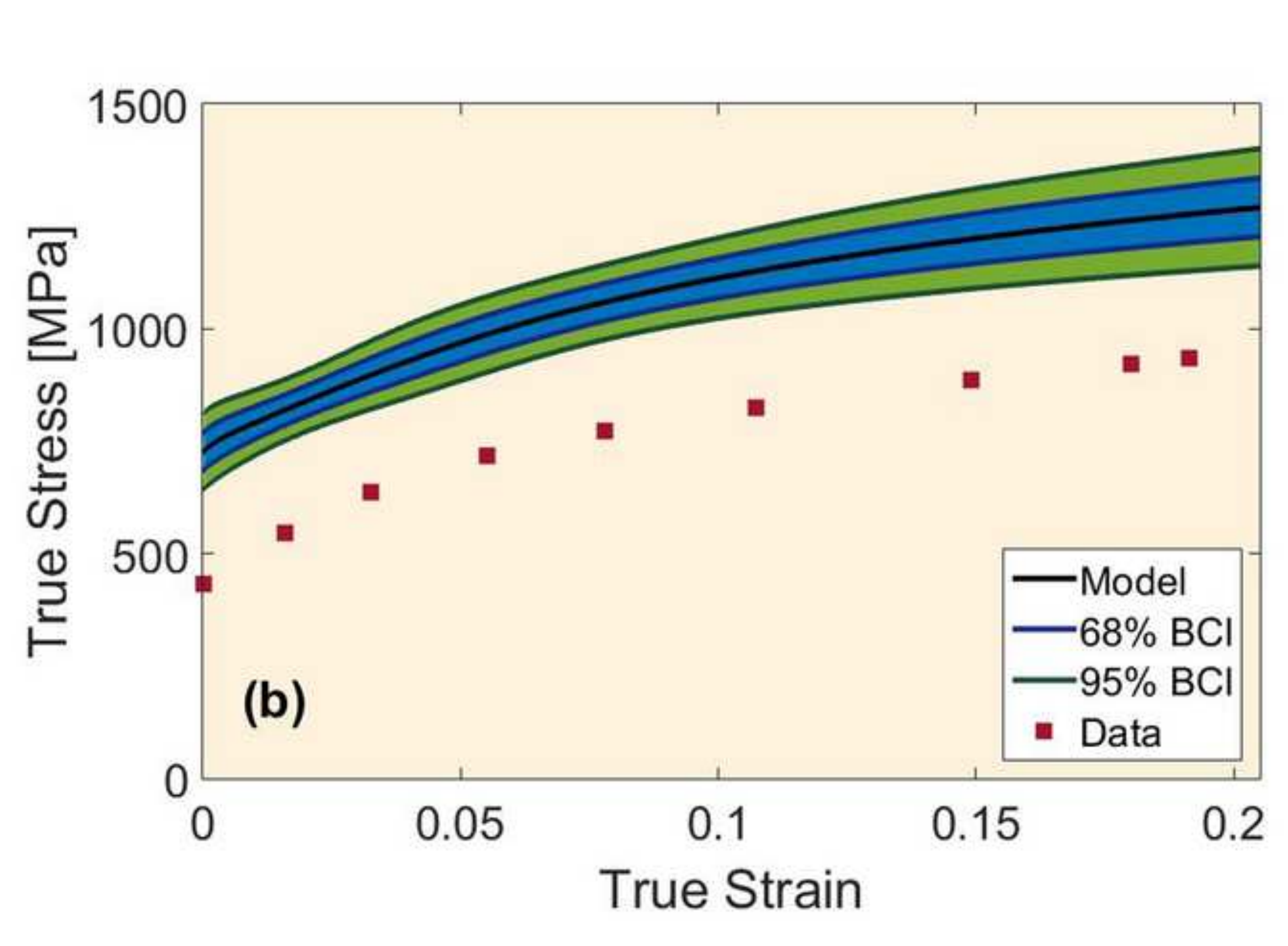

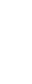

\section{True Strain}
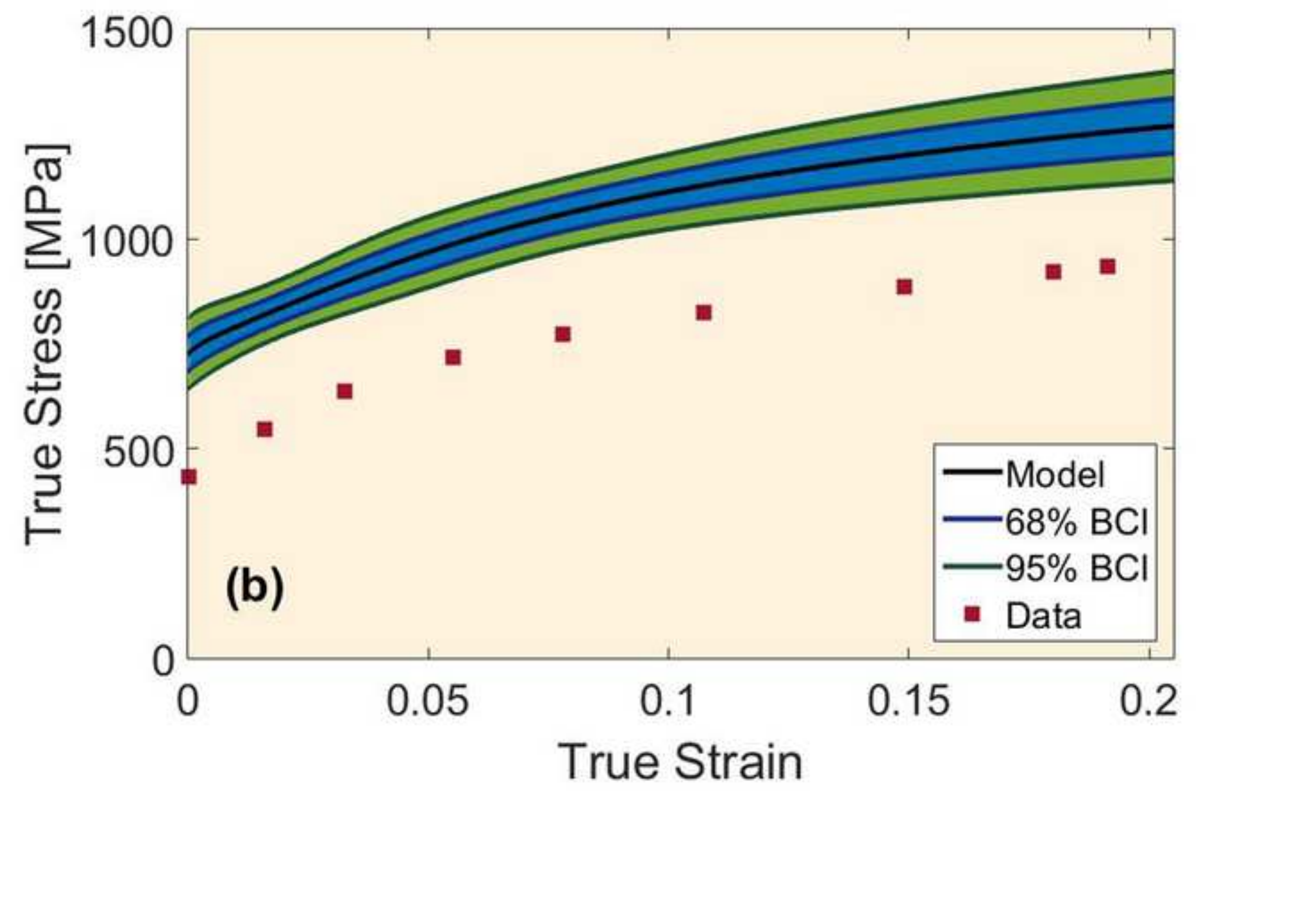

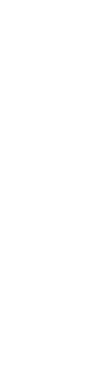

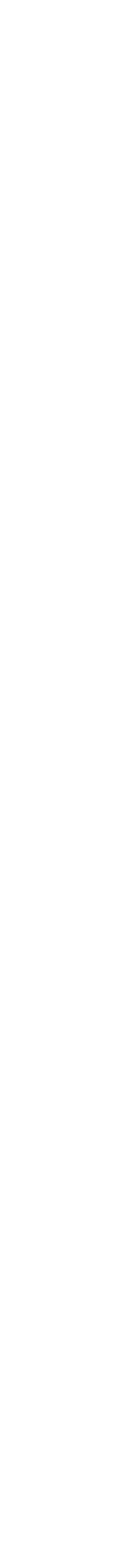

\section{True}




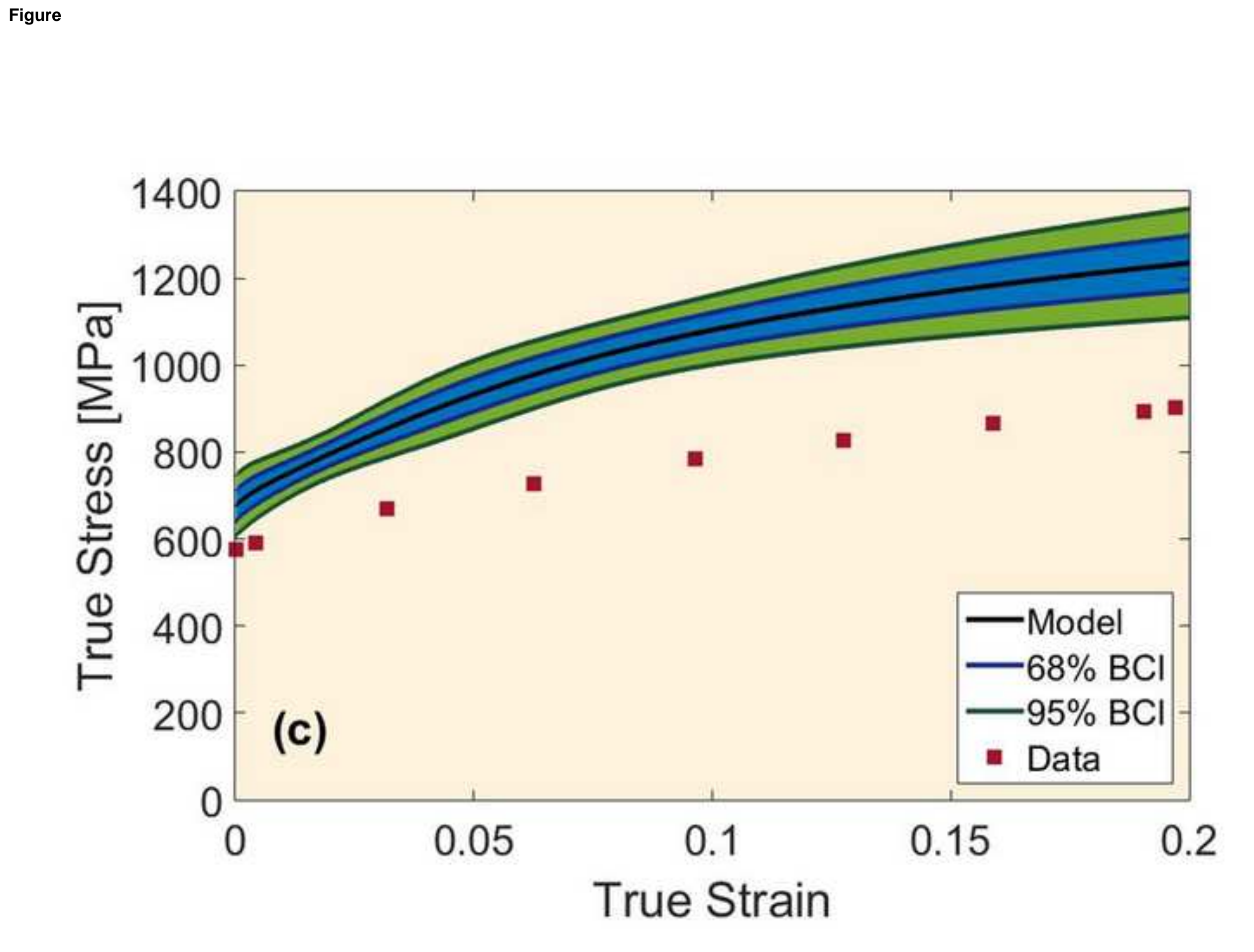

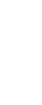

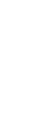

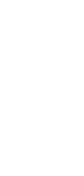

(n)

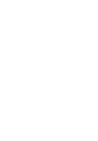

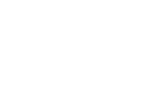

True Strain

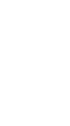
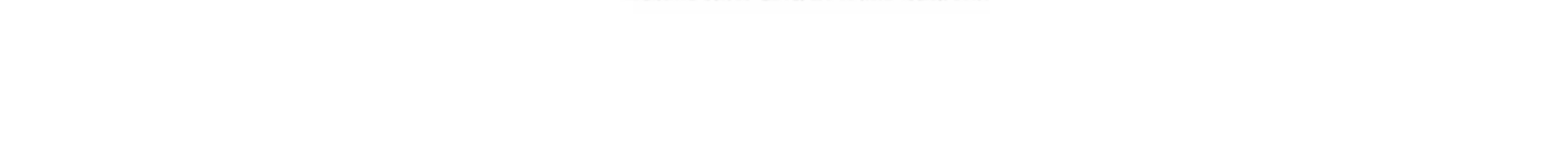

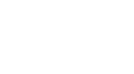

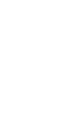




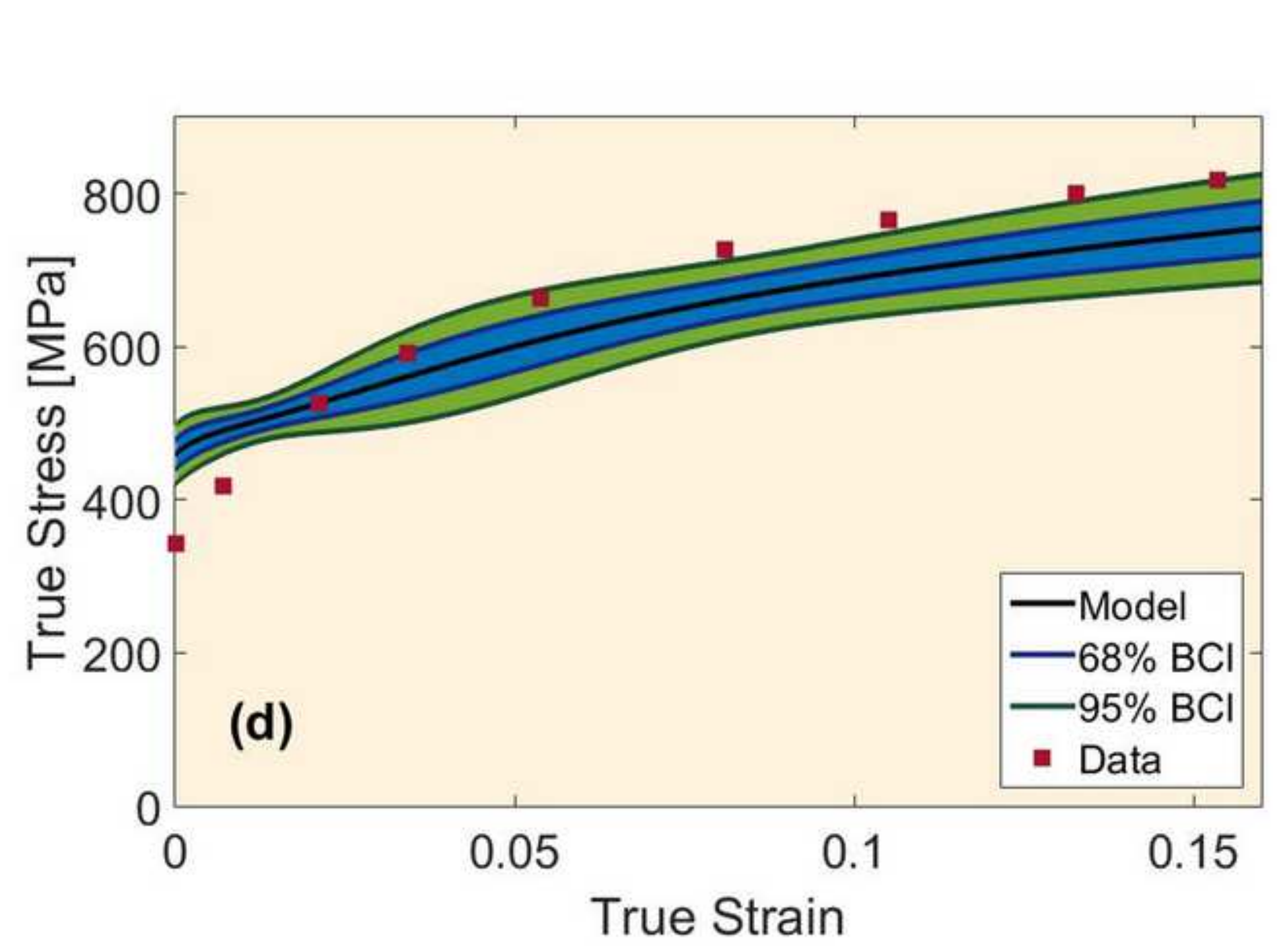




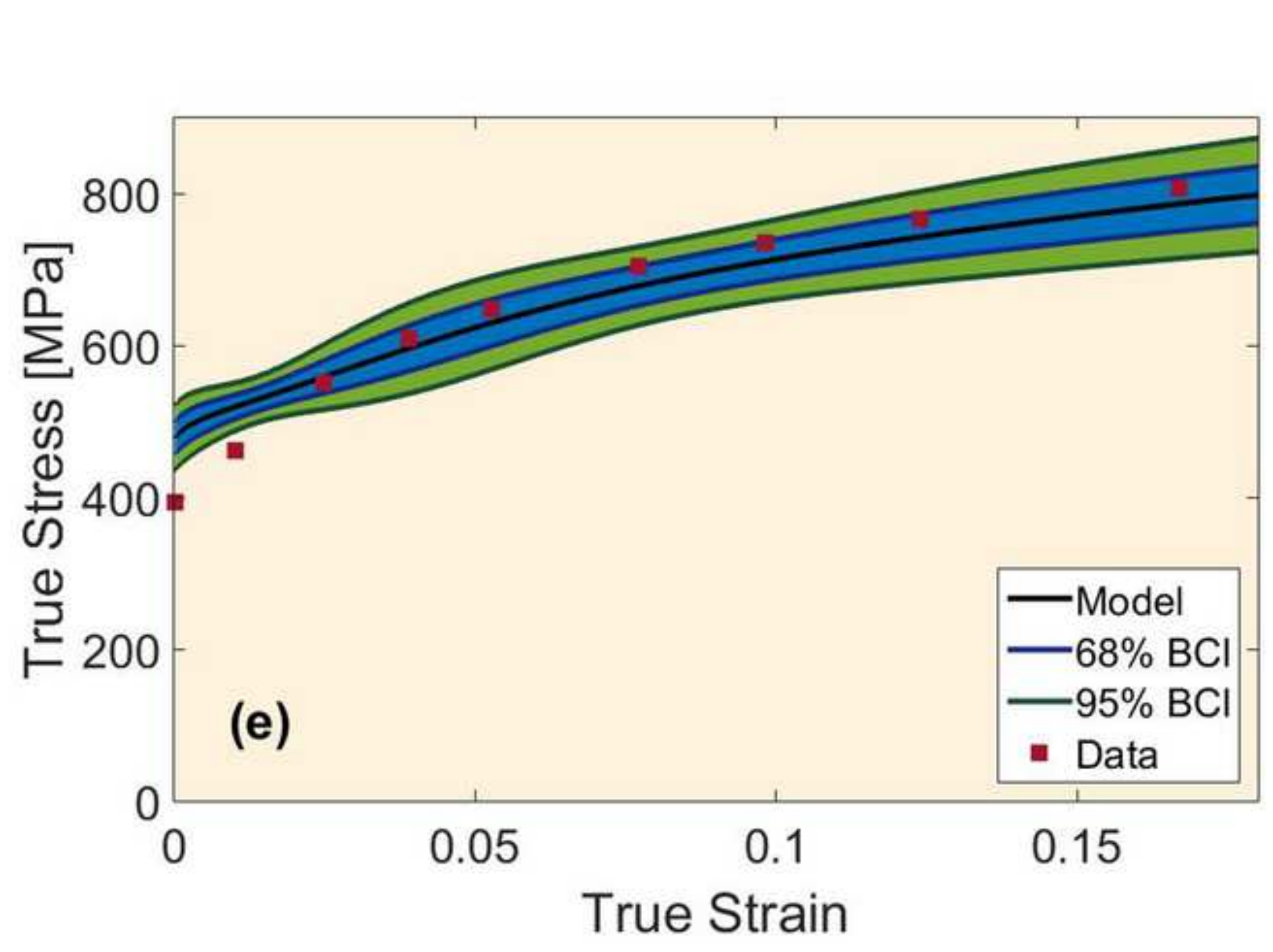



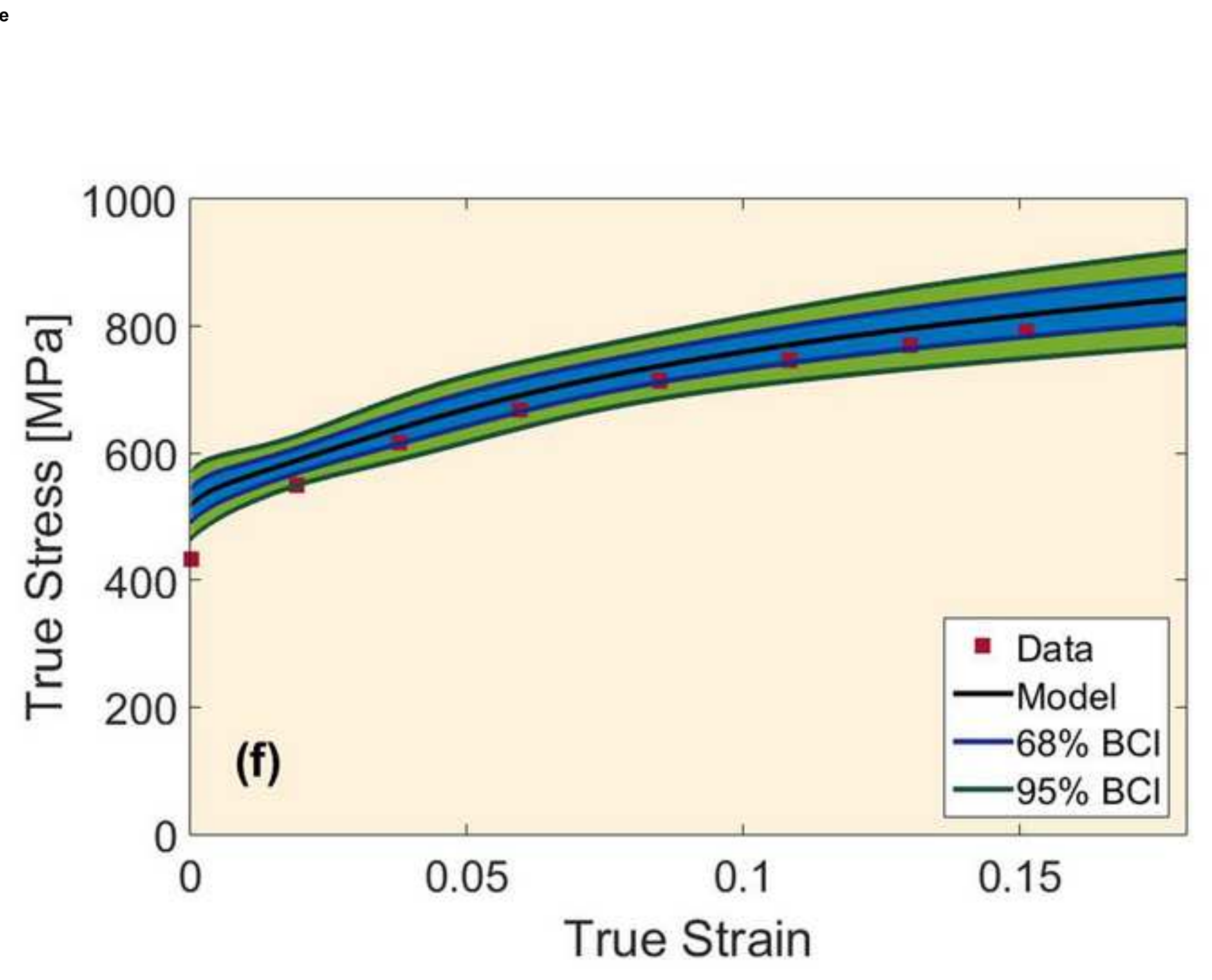

\section{True Strain}

(1)

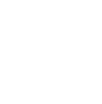
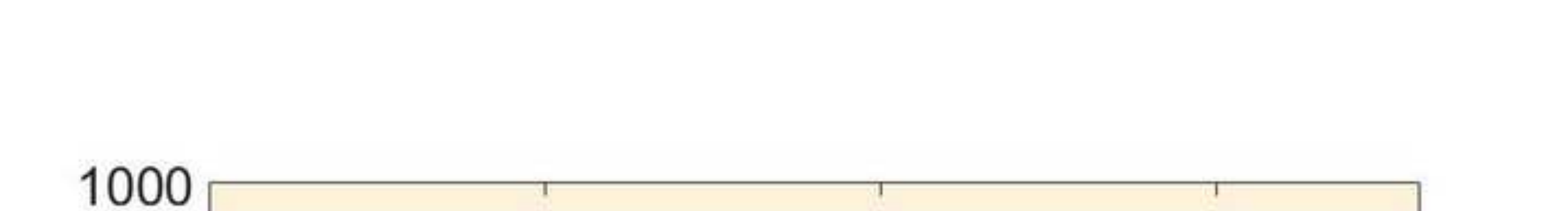

列
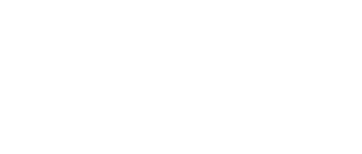

西

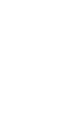




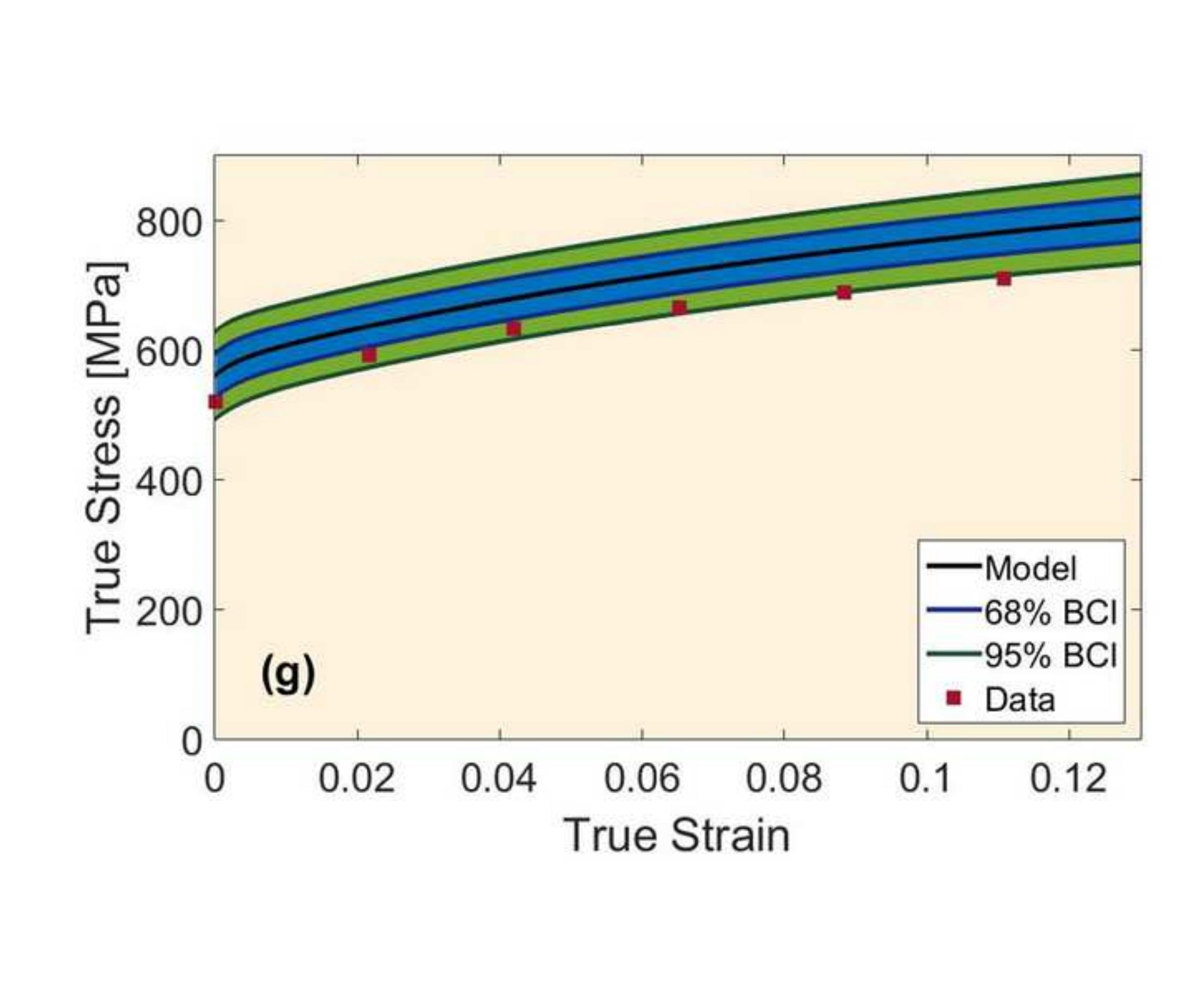

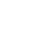

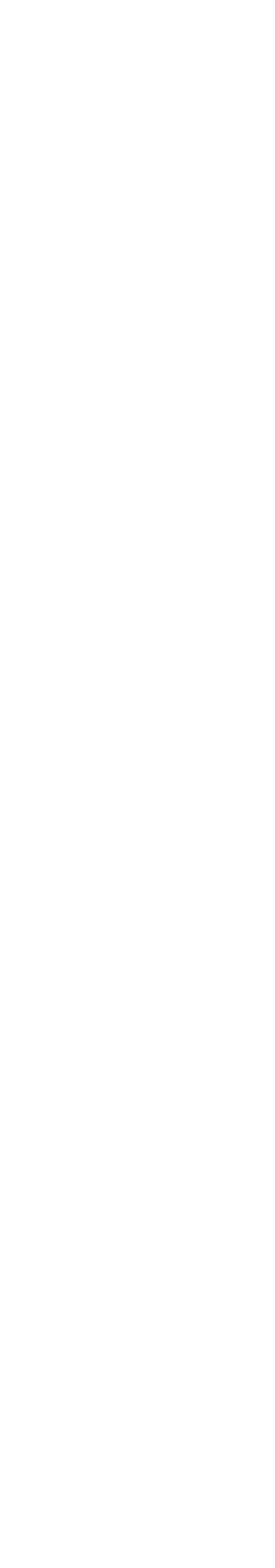

.

.

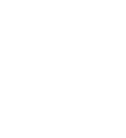

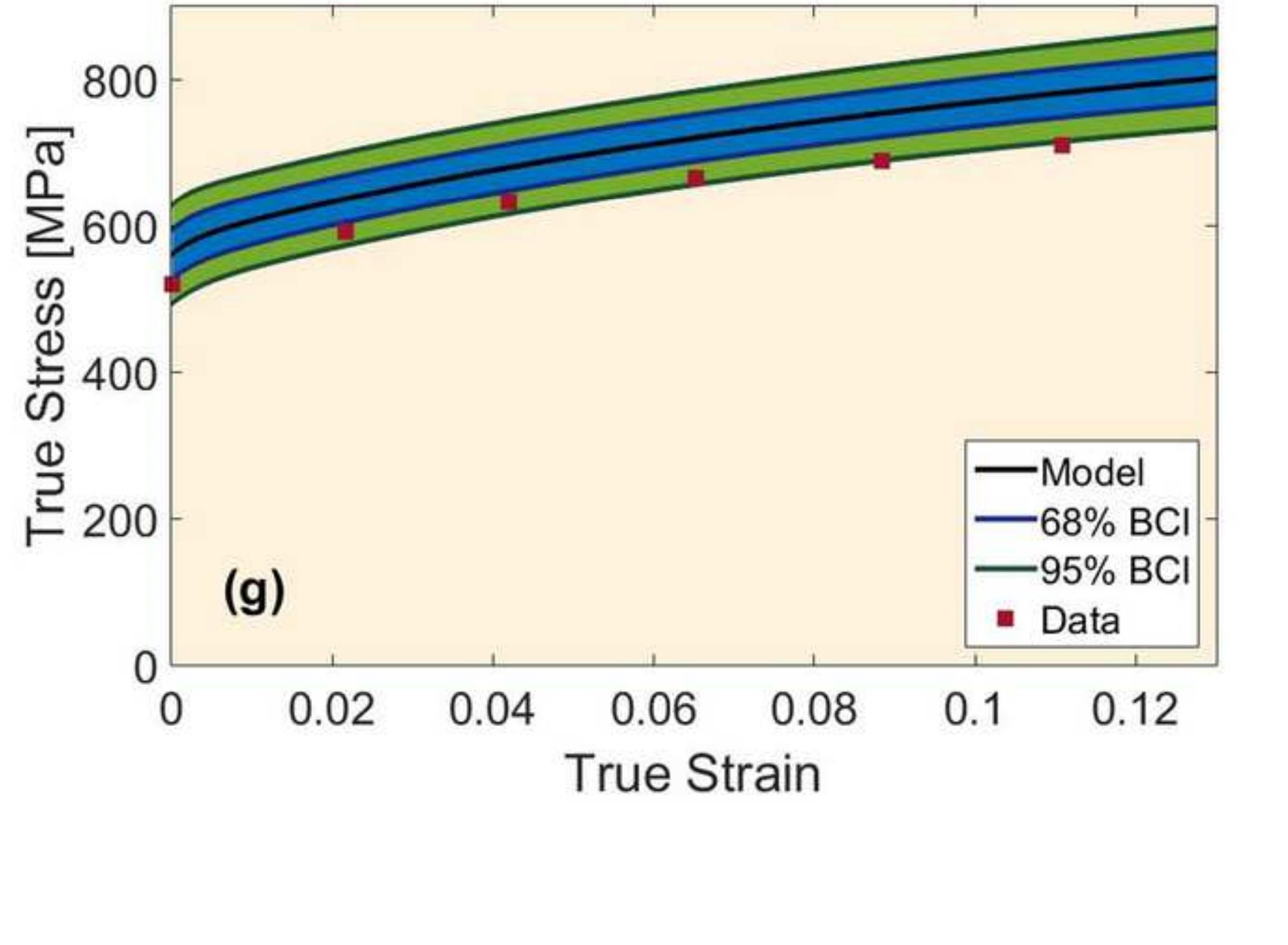

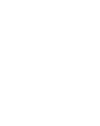

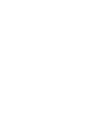

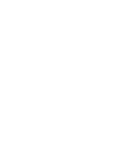



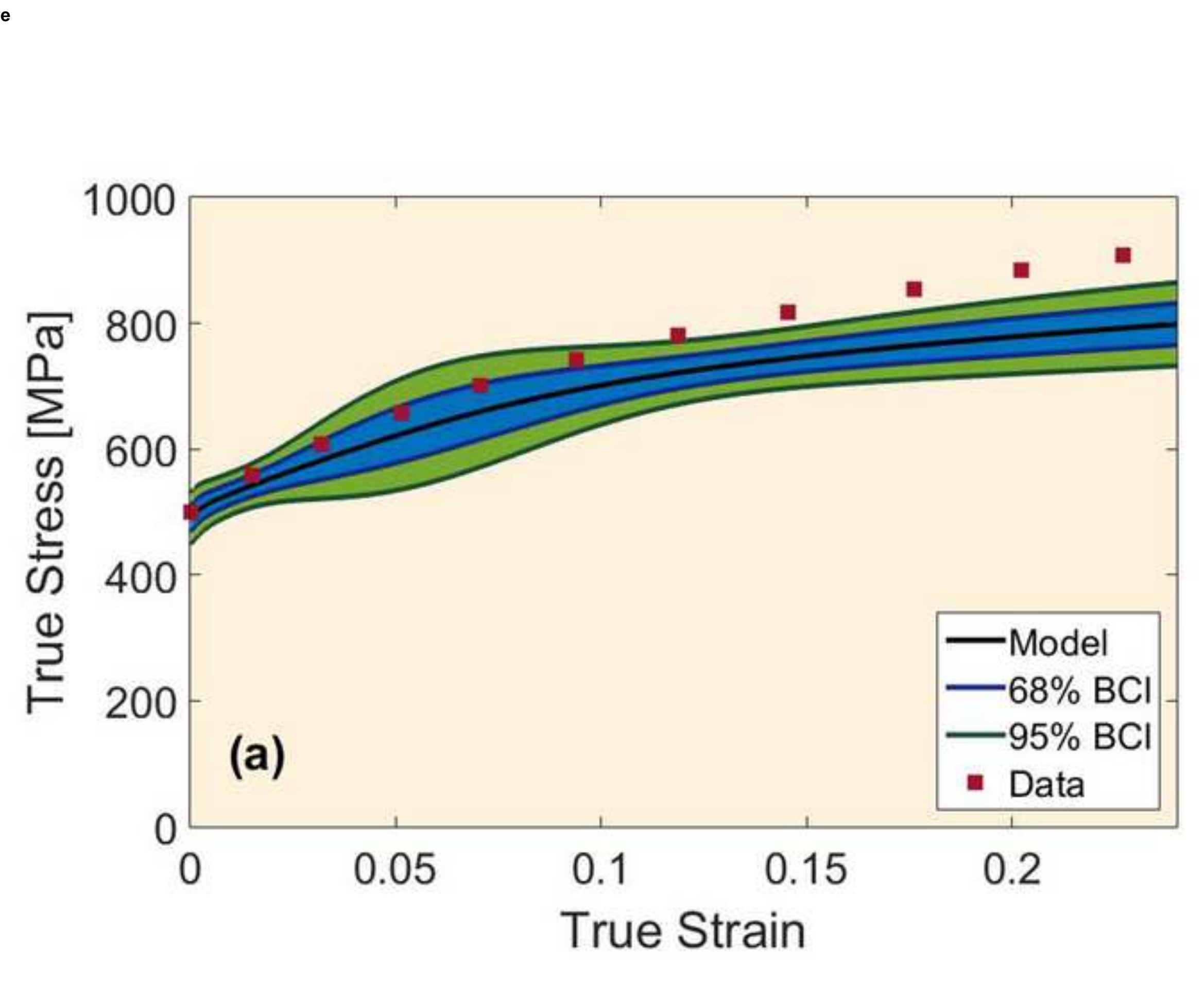

\section{True Strain}

.

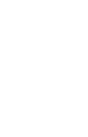

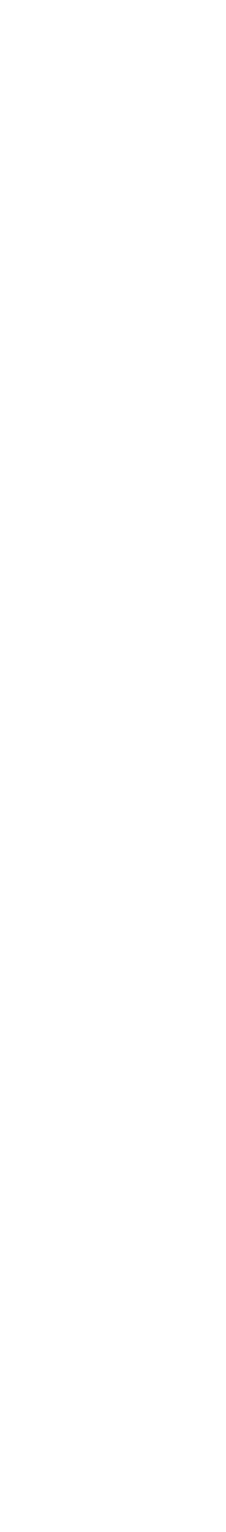

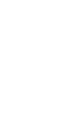




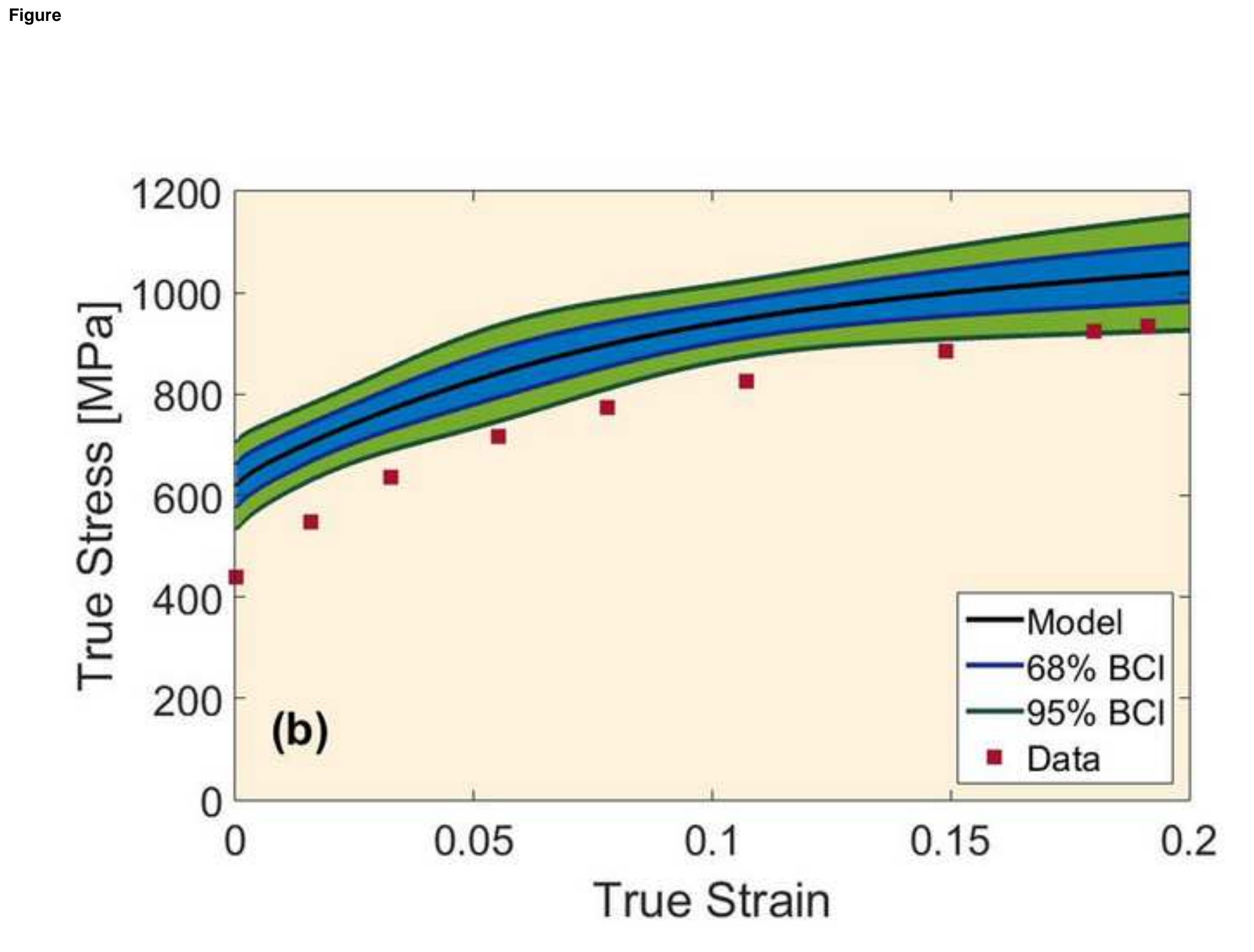

.

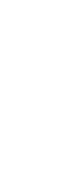

(

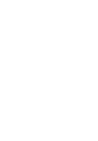

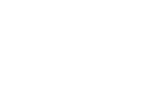

True Strain
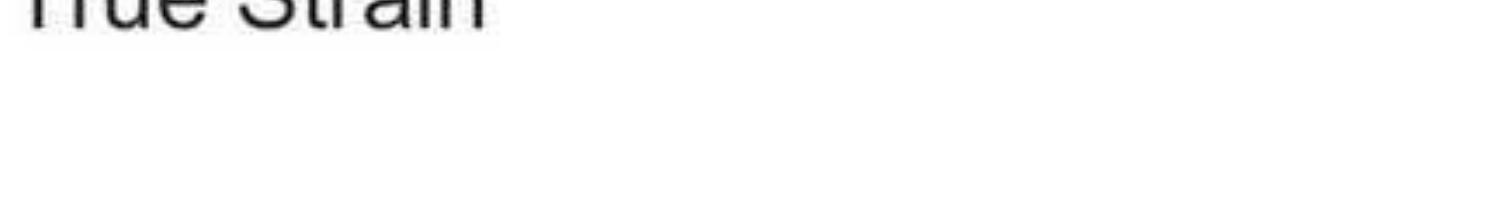


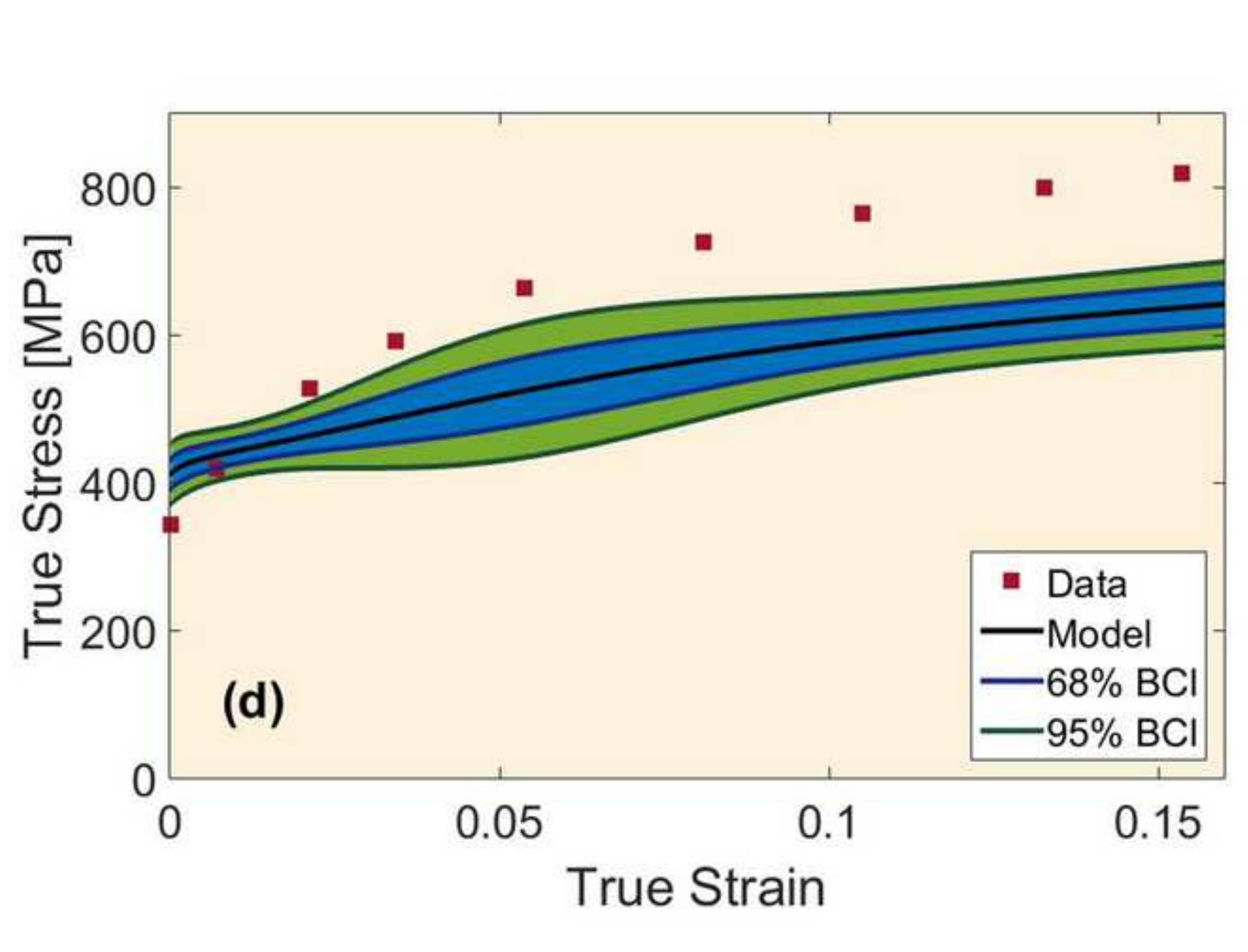

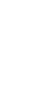

.

\section{True Strain}
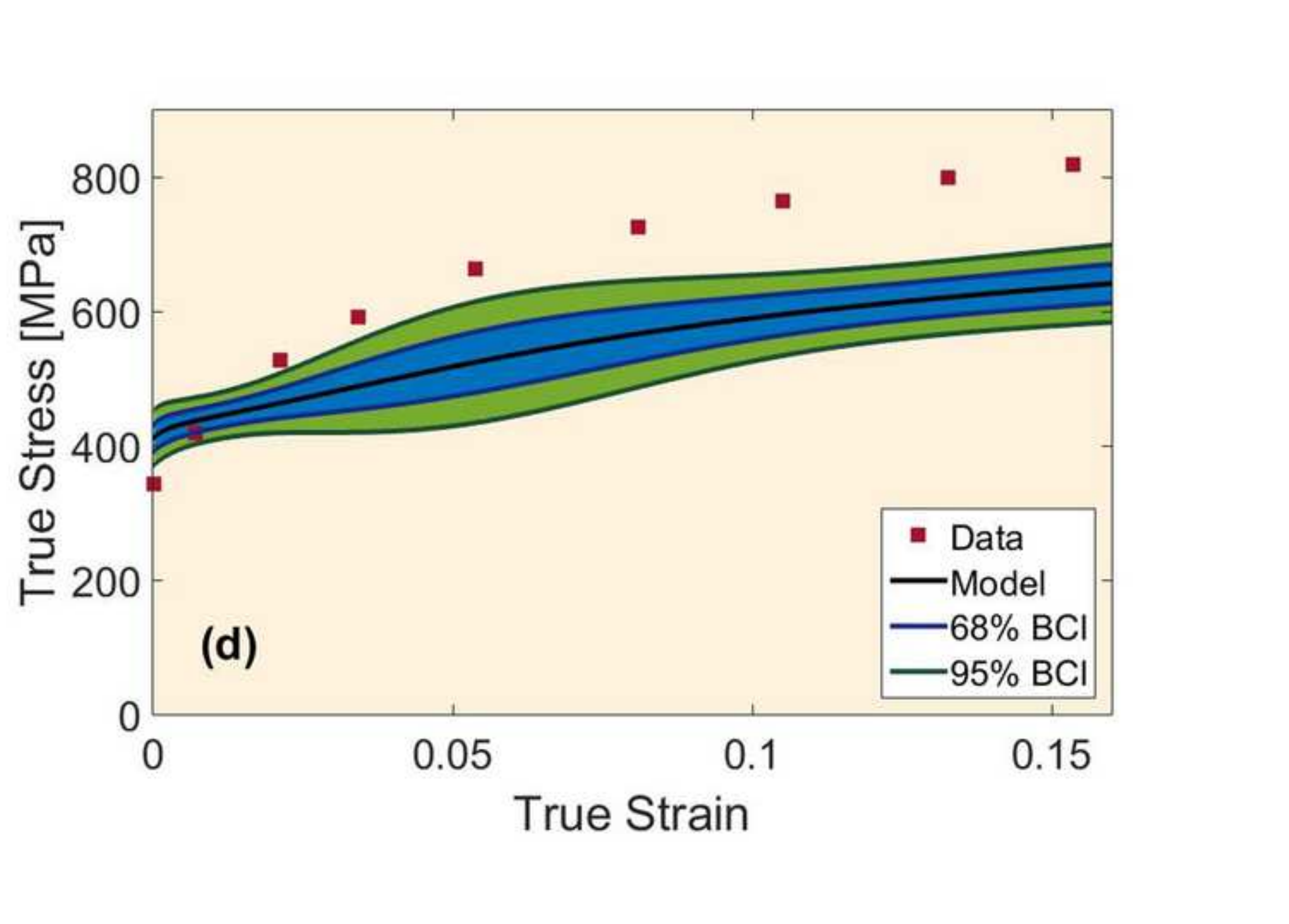


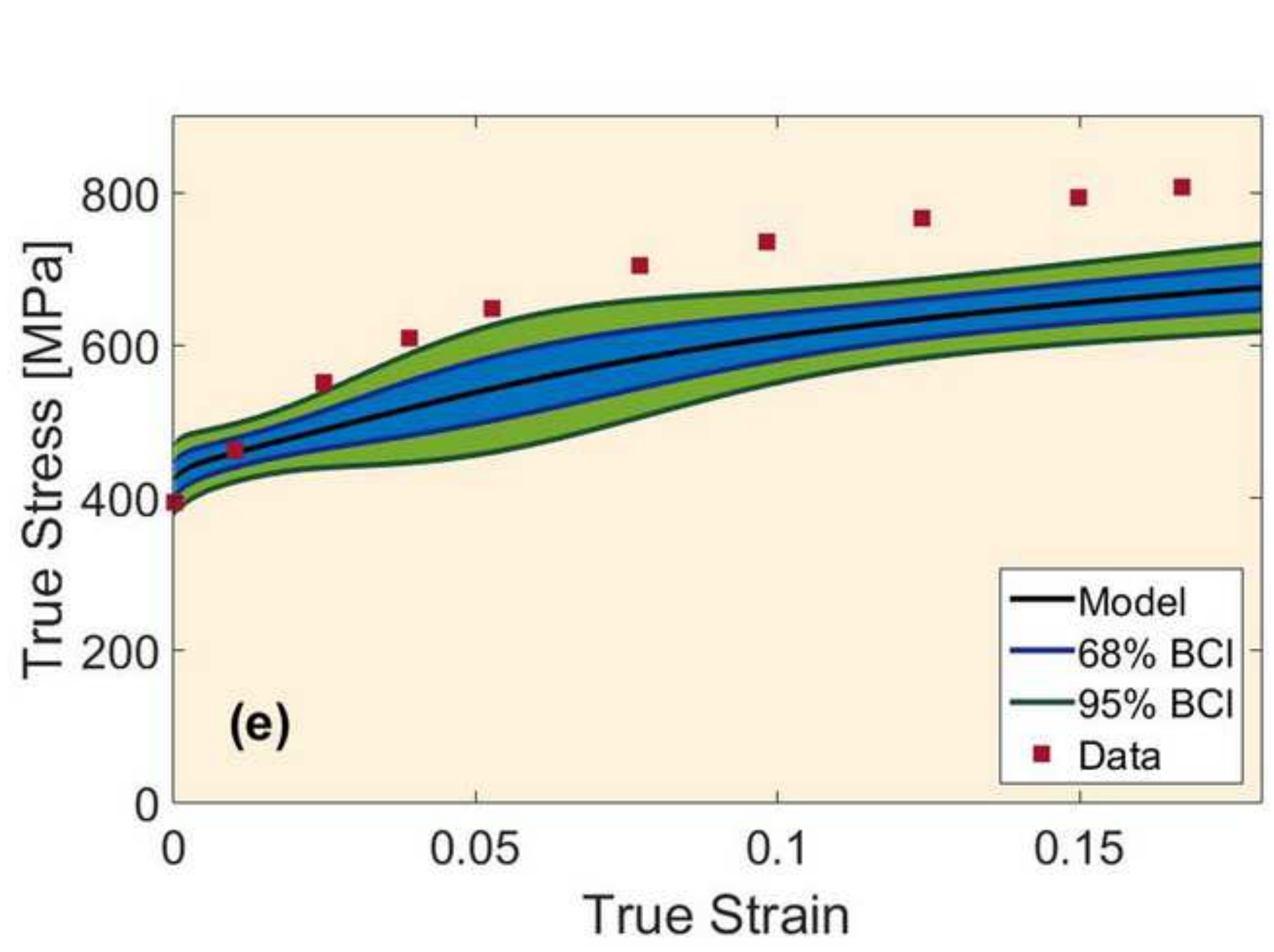




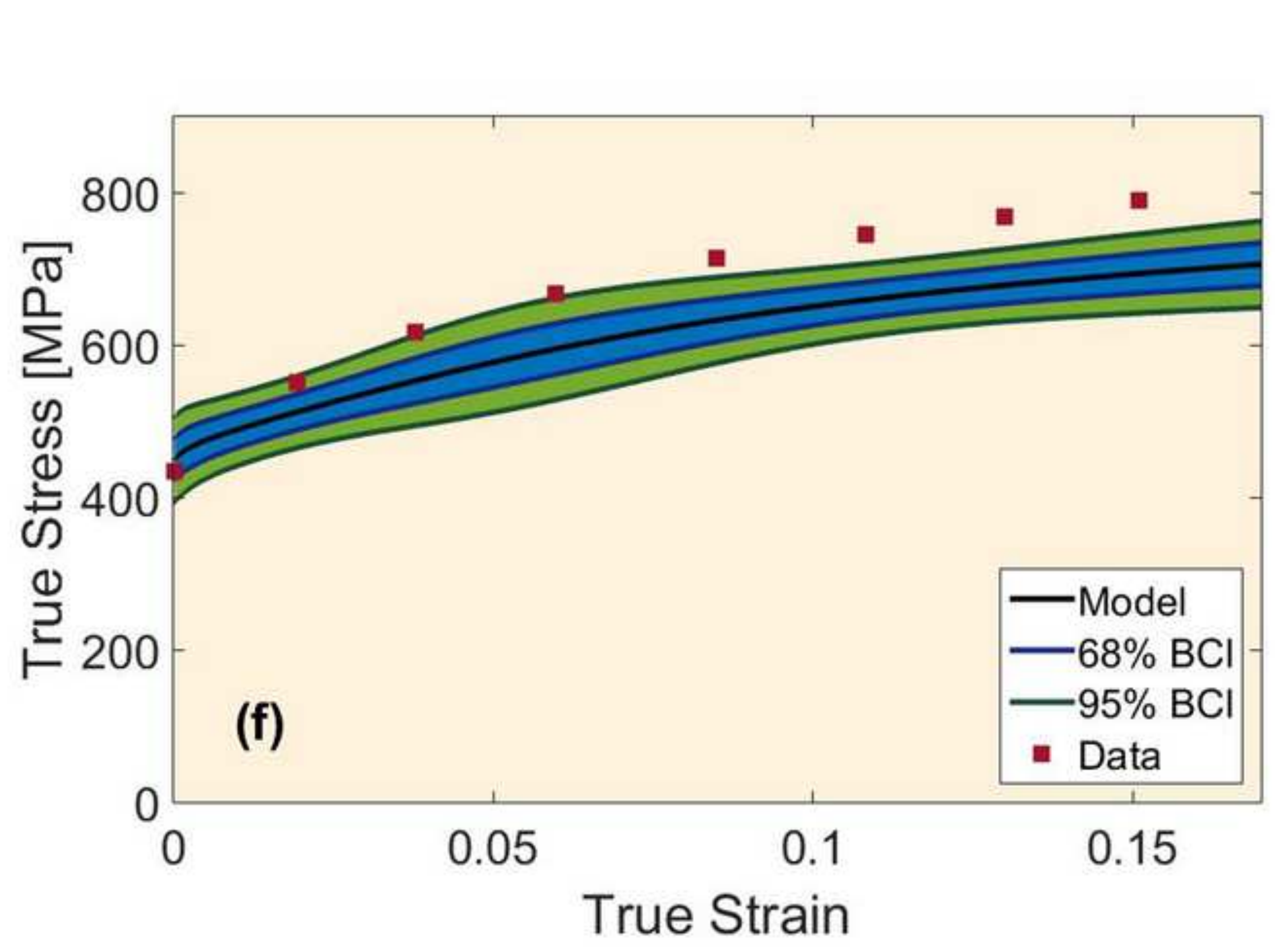




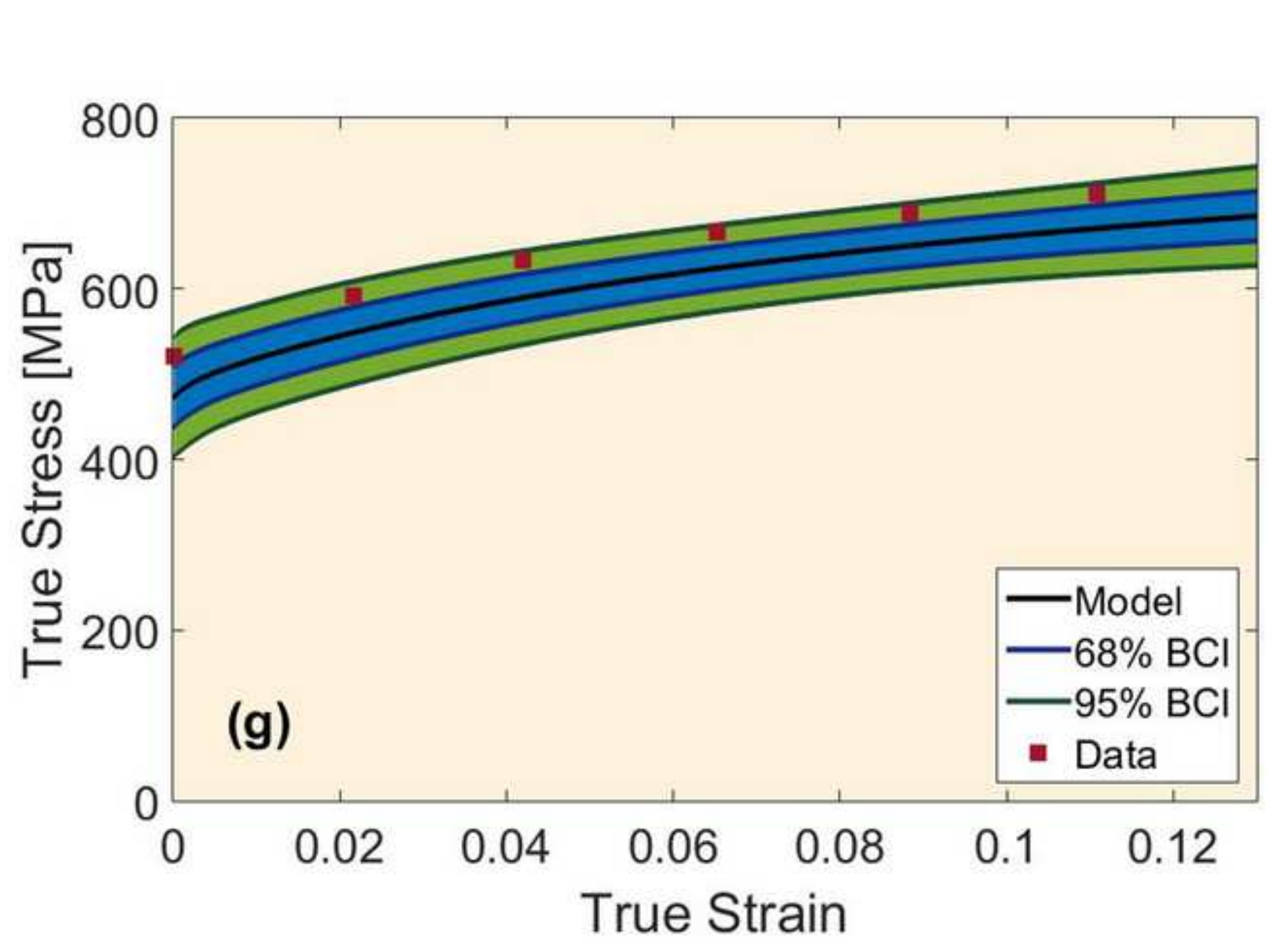




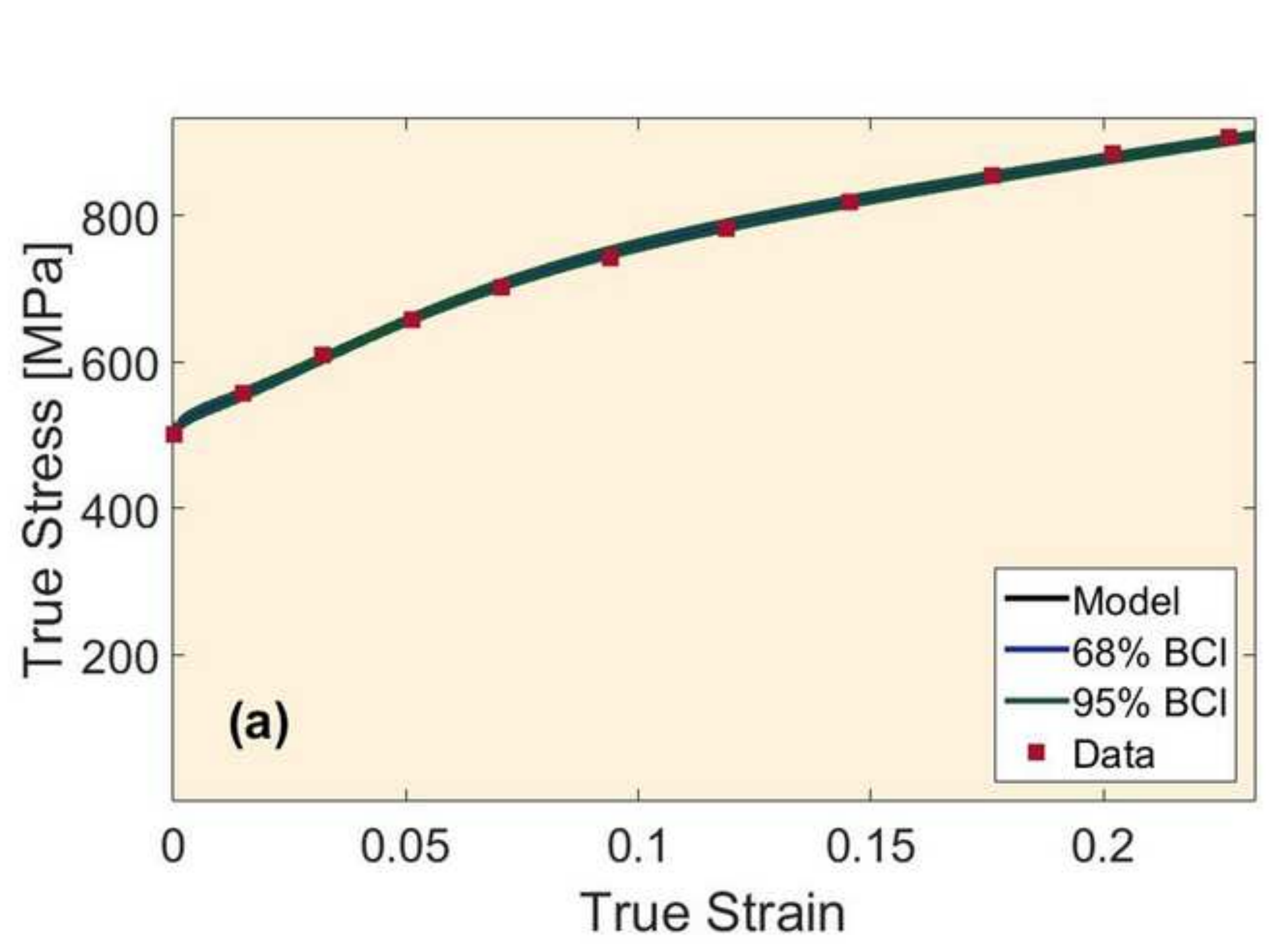




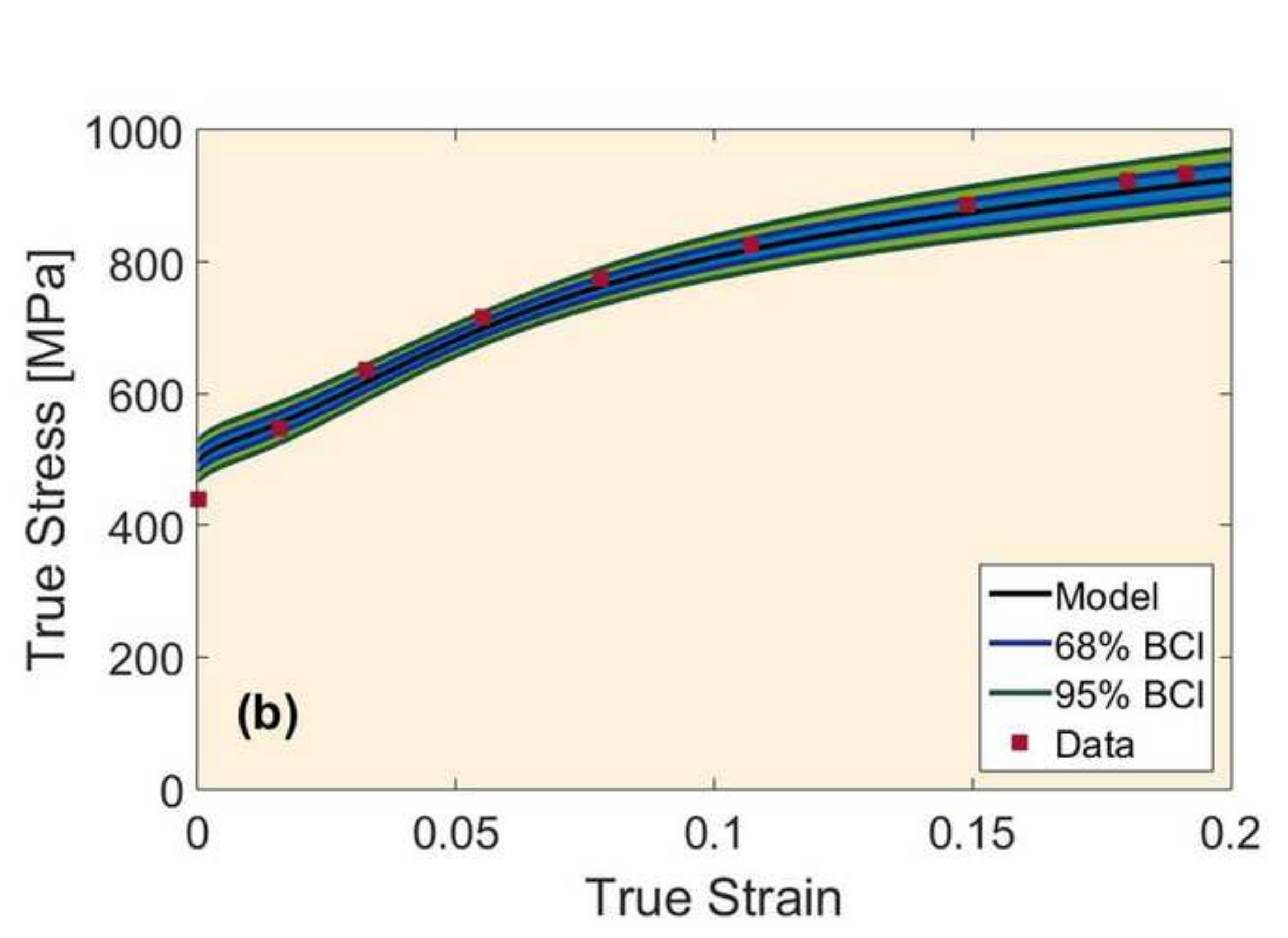

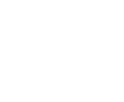

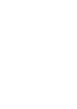

.

( 


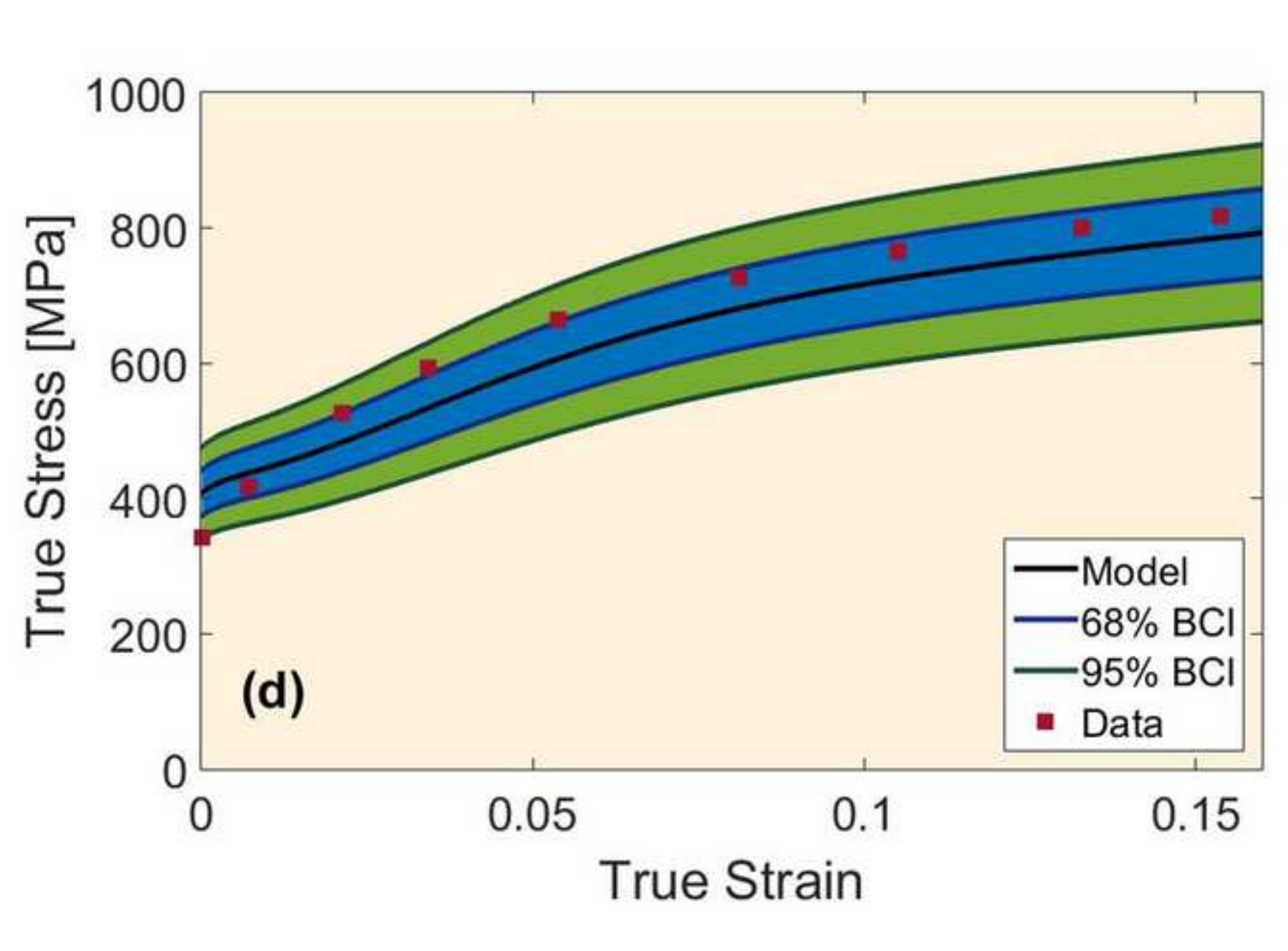

.

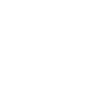

\section{True Strain}

.

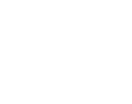

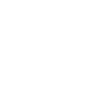

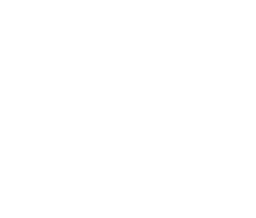




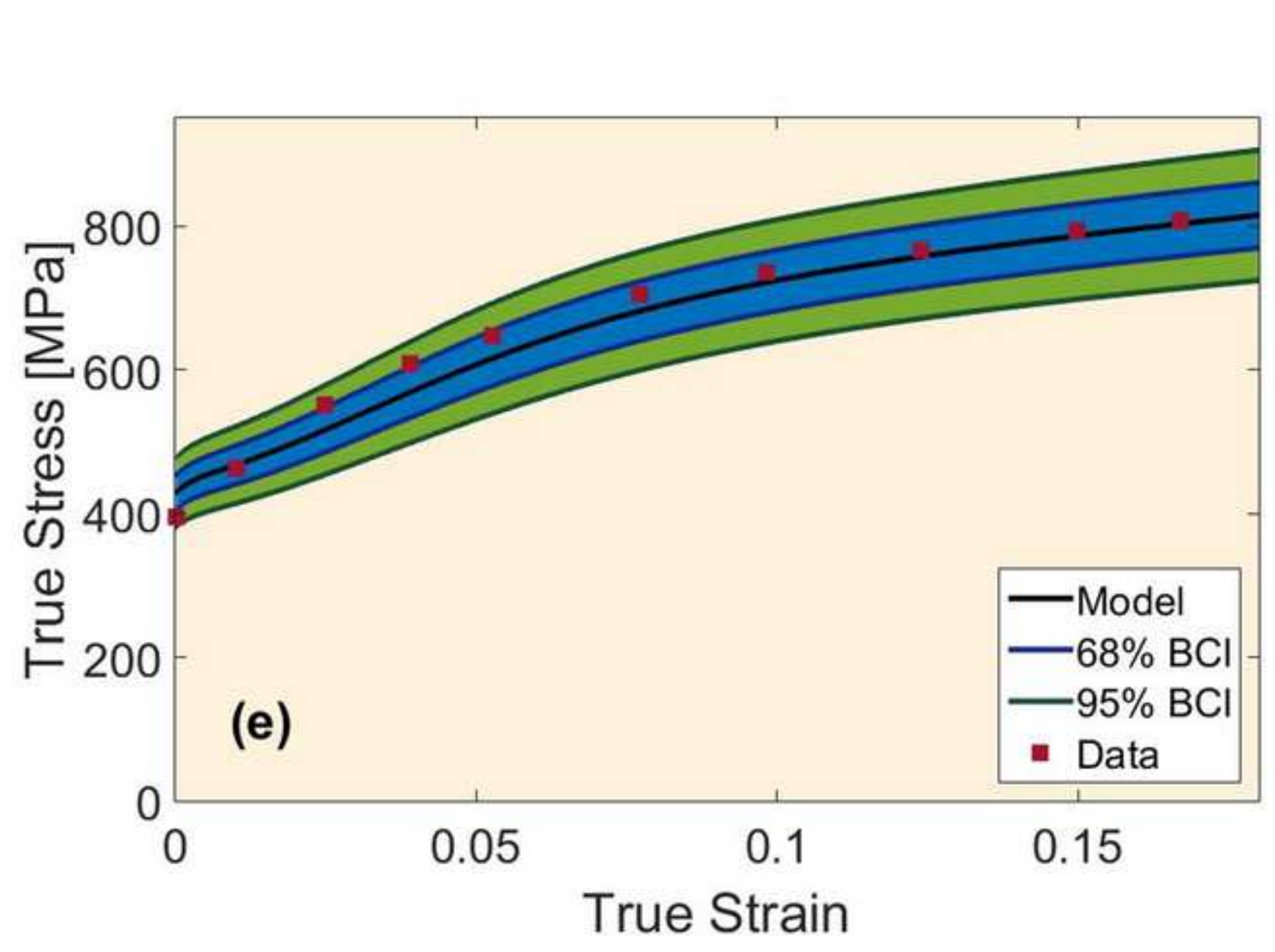




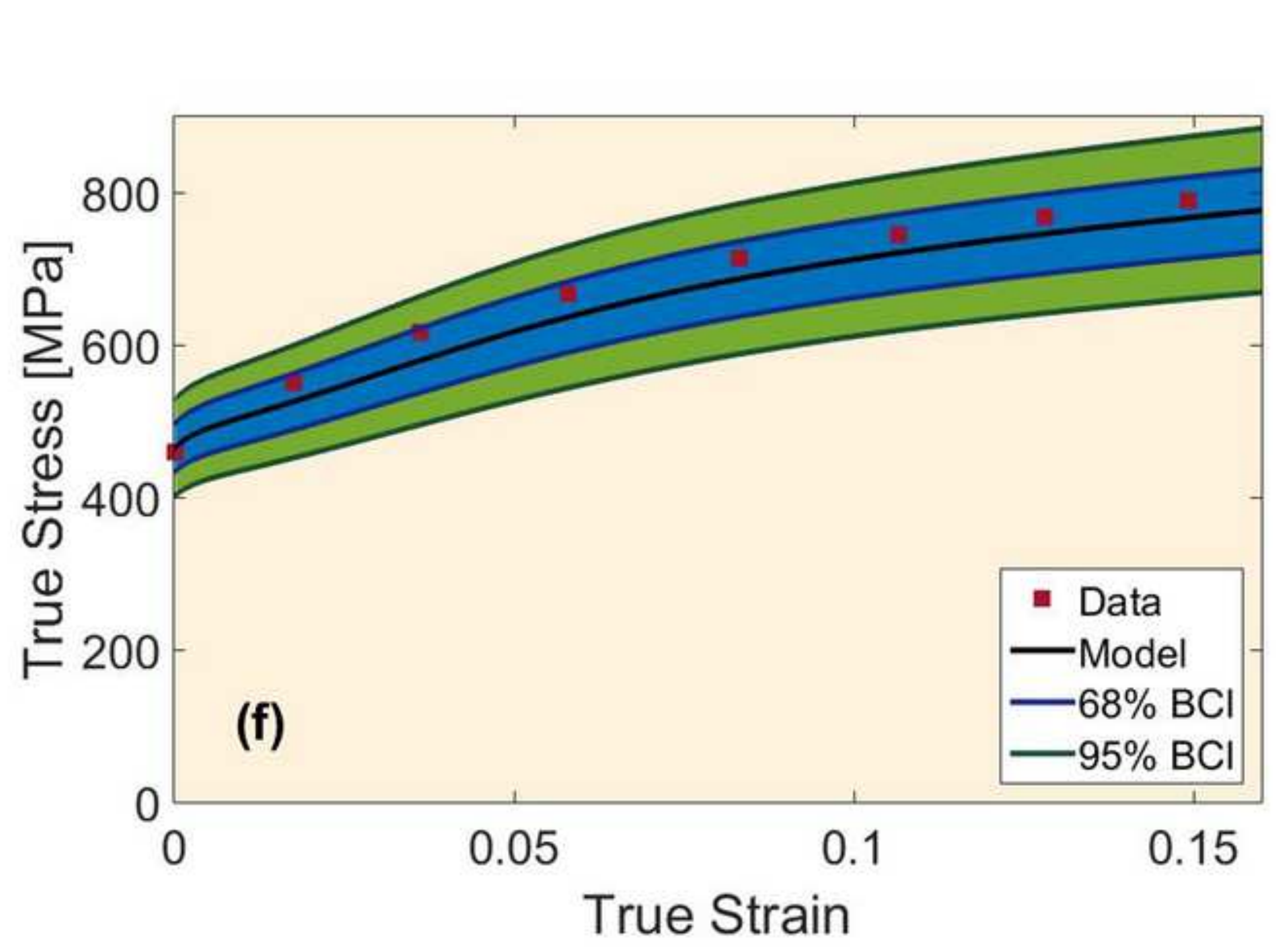




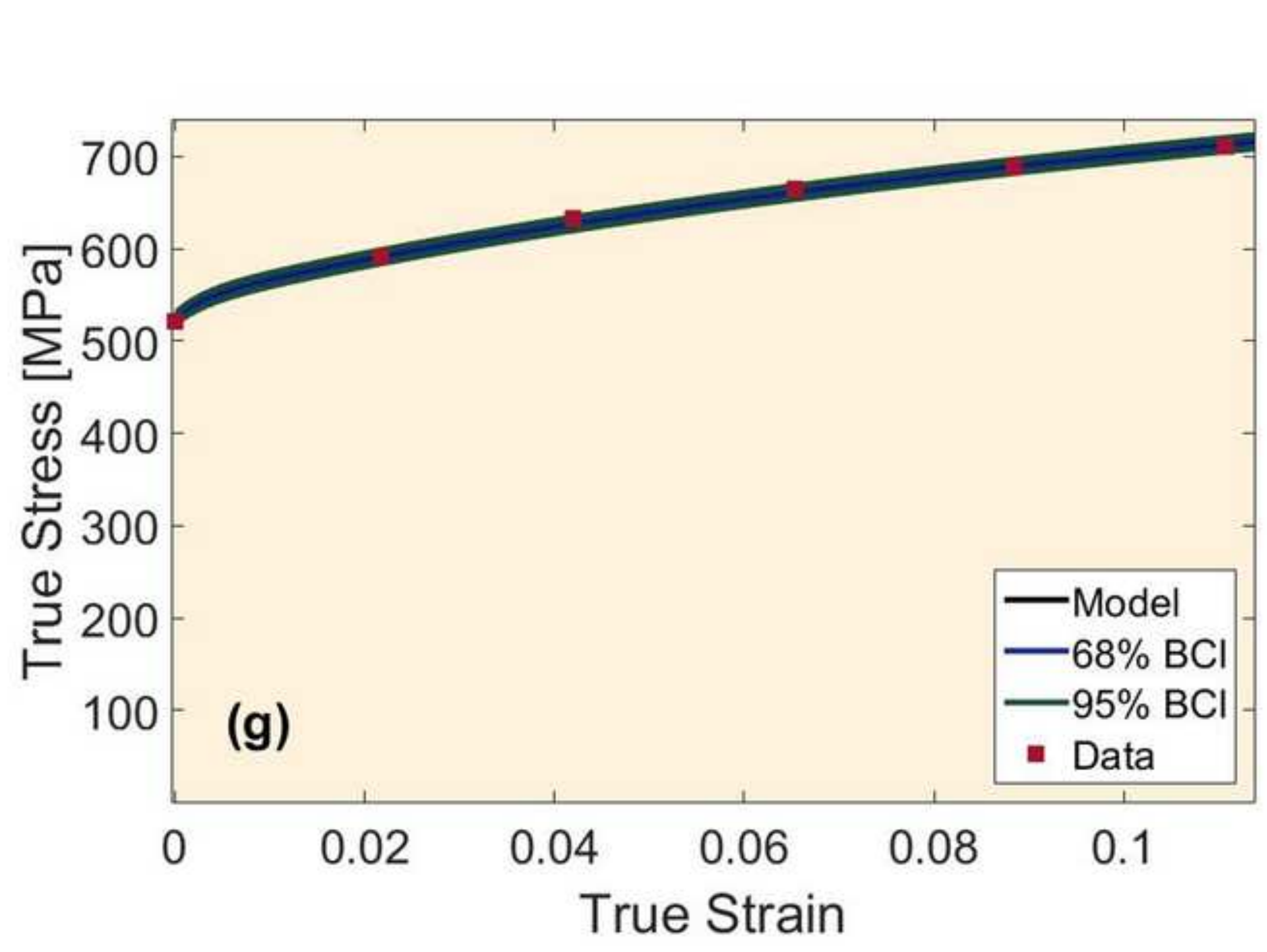




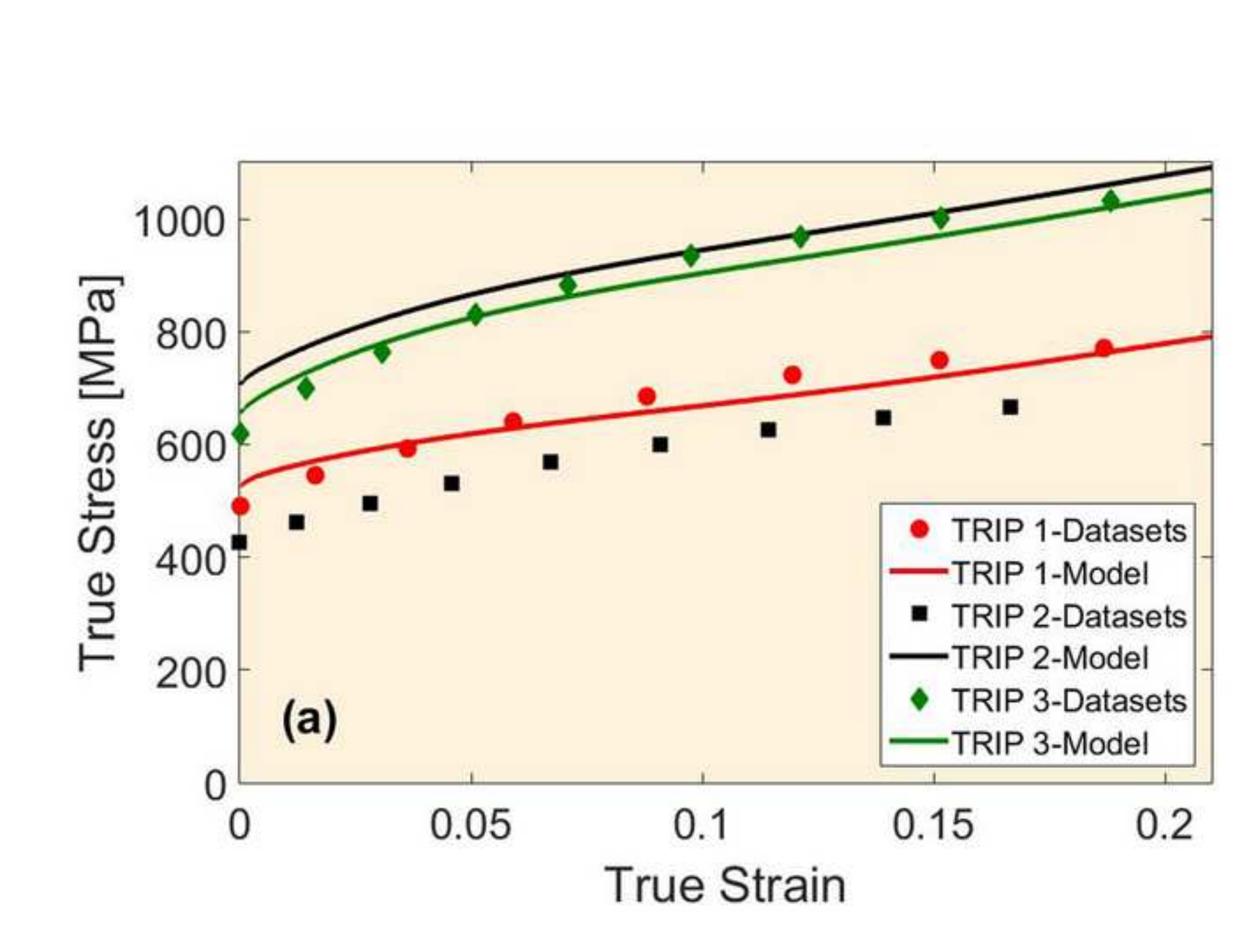

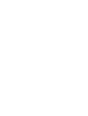

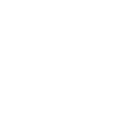




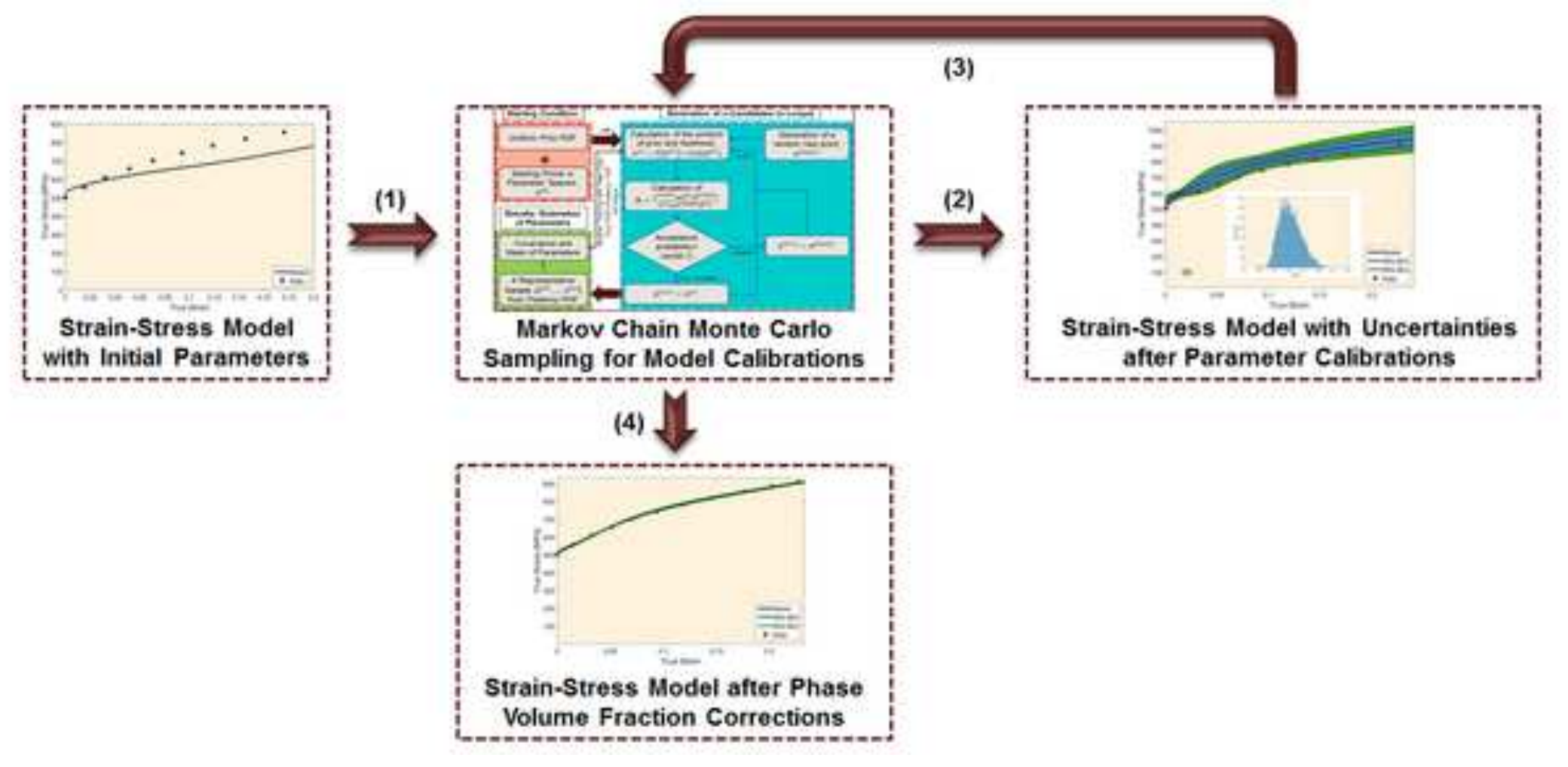

Volume Fraction Corrections 